\title{
Non-invasive Assessment and Compensation of Soft-tissue Artefacts in Hip Joint Kinematics
}

\author{
By
}

\section{Azadeh Rouhandeh}

A thesis submitted to the Faculty of Graduate and Postdoctoral Affairs in partial fulfillment of the requirements for the degree of

Master of Applied Science in Biomedical Engineering

Ottawa-Carleton Institute for Biomedical Engineering Department of Systems and Computer Engineering

Carleton University

Ottawa, Ontario

(C) 2014, Azadeh Rouhandeh 


\begin{abstract}
Accurate location of the hip joint centre is a necessary component in biomechanical human motion analysis to measure skeletal parameters and describe human motion. In human movement analysis, the hip joint centre can be estimated using functional methods based on the relative motion of the femur to pelvis using reflective markers attached to the skin surface through an optical motion capture system. Determination of the hip joint centre by functional methods suffers inaccuracy due to the soft tissue artefact; this is the relative motion between the markers and the underlying bone due to the muscle and skin deformation. Therefore, one of the main objectives in human movement analysis is the assessment and correction of this artefact. Various studies have described the movement of the soft tissue artefact and minimized it invasively. To solve this issue, we present a non-invasive method to assess and reduce the effect of the soft tissue artefact using optical motion capture data and tissue thickness from ultrasound measurements during flexion, extension, and abduction of the hip joint. Results show that the displacement of markers is non-linear and larger in areas closer to the hip joint. Also, the marker displacements are dependent on the movement type, being relatively larger in abduction movement. The quantification of soft tissue artefacts is used as a basis for a correction procedure for hip joint centre and minimizing the soft tissue artefact effects. Results show that our method for soft tissue artefact assessment and minimization reduces the error in the functional hip joint centre approximately from $13-23 \mathrm{~mm}$ to $7-14 \mathrm{~mm}$.
\end{abstract}




\section{Acknowledgements}

First of all, I would like to express my deepest gratitude to my supervisor Professor Chris Joslin for his excellent guidance and advice. Without his continuous support and assistance, the completion of this thesis would not have been possible.

I would like to thank Professor Yuu Ono and Mr Zhen Qu for their support of this work and valuable suggestions.

Last, but not least, I would like to extend my deepest thanks to those whose support cannot be forgotten, my family and especially my husband Ehsan, for their endless support in all my academic and personal endeavors. 


\section{Table of Contents}

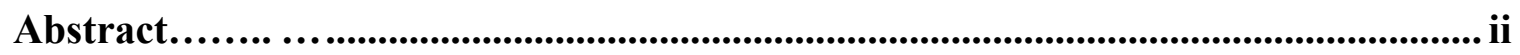

Acknowledgements ............................................................................................................................. iii

Table of Contents .............................................................................................................................. iv

List of Figures ............................................................................................................................ vii

List of Tables.....................................................................................................................

Abbreviations ................................................................................................................

Chapter 1: Introduction ................................................................................... 1

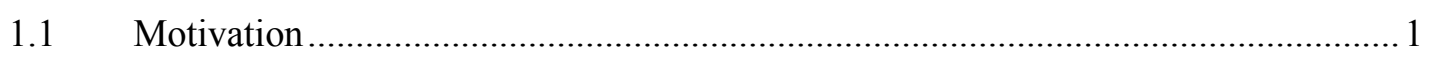

$1.2 \quad$ Objectives and Contributions ...........................................................................

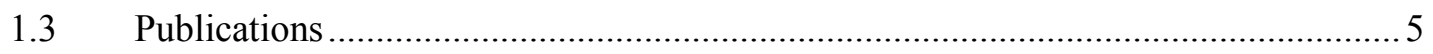

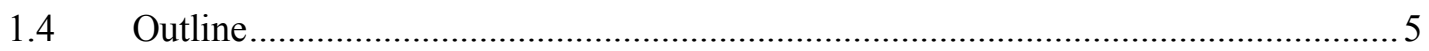

Chapter 2: Introduction to the Hip Joint .................................................................. 7

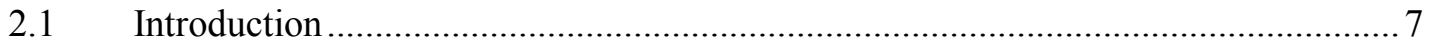

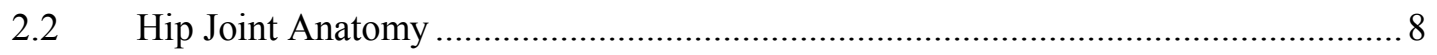

2.3 Clinical Movements of the Hip Joint ………………………………………….... 10

2.4 Techniques for Human 3D Motion Recording .................................................... 12

2.4.1 Bone-based Techniques..................................................................... 12

2.4.2 Skin-based Techniques........................................................................ 15

Chapter 3: $\quad$ Related Studies ................................................................................................. 17

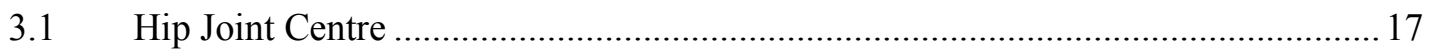

3.1.1 Image-based Techniques ................................................................... 18

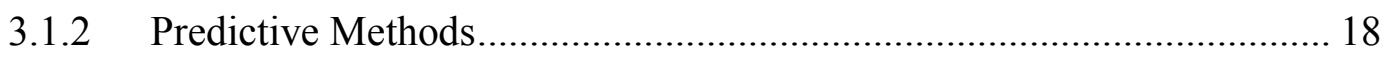

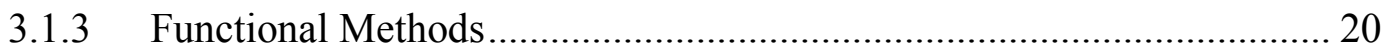




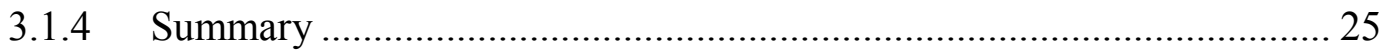

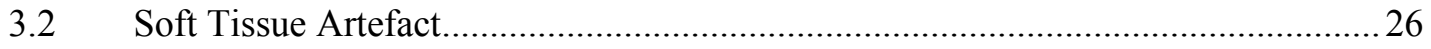

3.3 Soft Tissue Artefact Assessment ....................................................................2

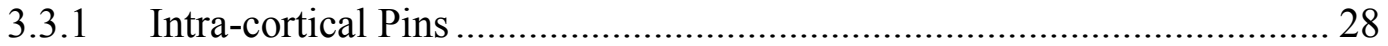

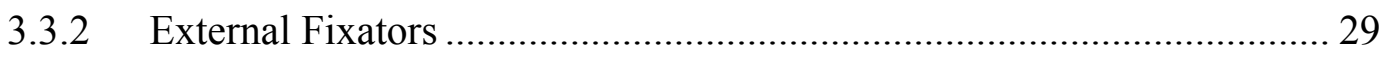

3.3.3 Percutaneous Trackers...................................................................... 29

3.3.4 Radiographic Examinations ………………….................................. 30

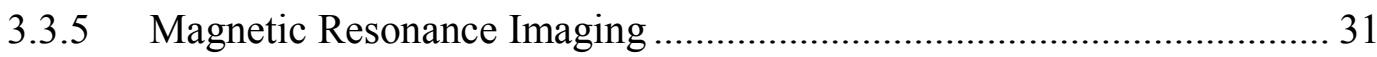

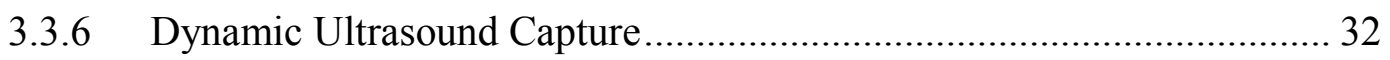

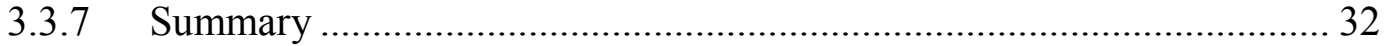

3.4 Soft Tissue Artefact Minimization.........................................................................

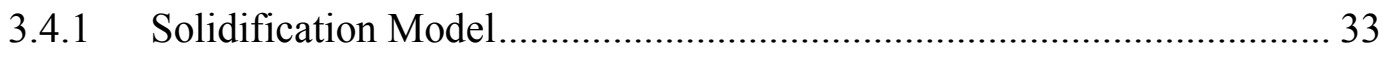

3.4.2 Multiple Anatomical Landmark Calibration ......................................... 34

3.4.3 Pliant Surface Modeling ....................................................................... 35

3.4.4 Dynamic Anatomical Landmark Calibration .......................................... 36

3.4.5 Point Cluster Technique ........................................................................ 36

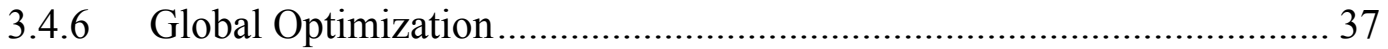

3.4.7 Magnetic Resonance Imaging .......................................................... 38

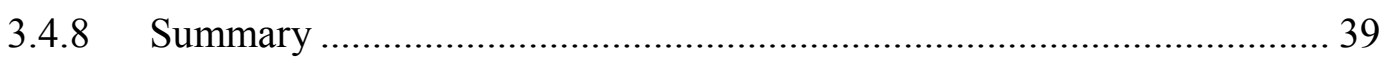

\section{Chapter 4: Developing a Method to Assess and Minimize Soft Tissue Artefact in} Hip Joint Centre Estimation ..................................................................................................... 41

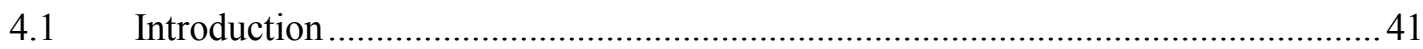

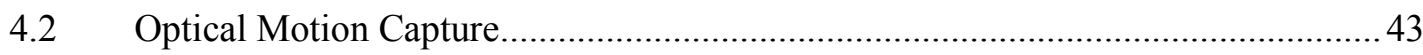

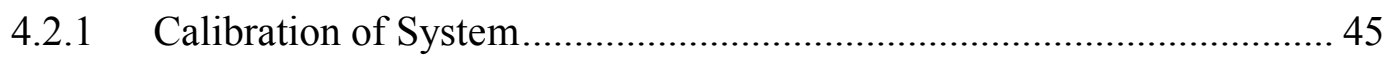

4.2.2 Markers Configuration ........................................................................ 46

4.2.3 Motion Tracking................................................................................ 47

4.2.4 Data Post-processing …………………………................................... 48

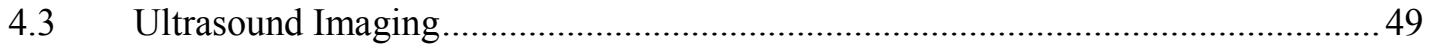

4.3.1 Edge Detection in Ultrasound Images................................................. 51

4.3.2 Determine Three Key Markers............................................................. 52

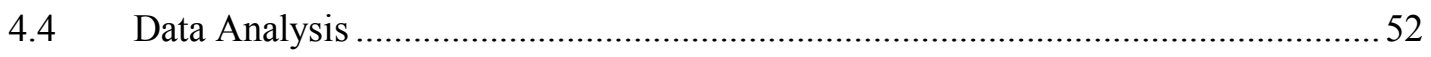




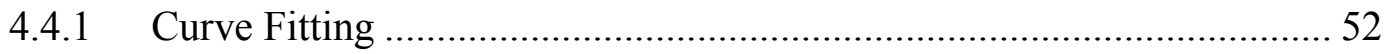

4.4.2 Define a Plane through the Curves...................................................... 54

4.4.3 Bone Position at Three Key Markers Positions .................................... 55

4.4.4 Transformation of the Three Key Markers .......................................... 56

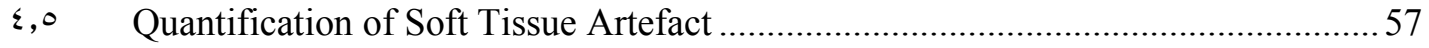

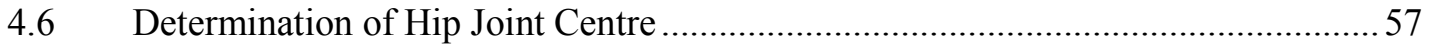

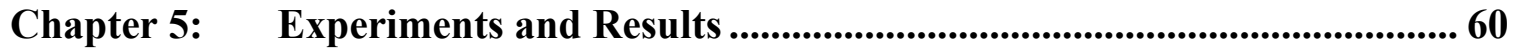

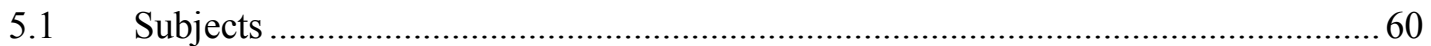

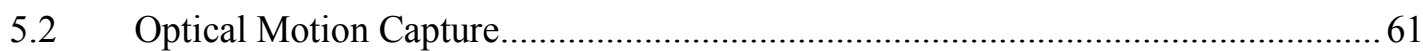

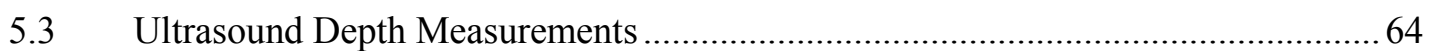

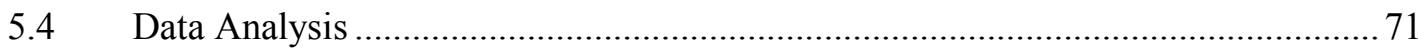

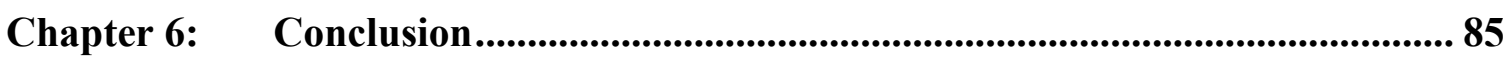

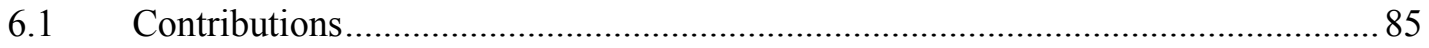

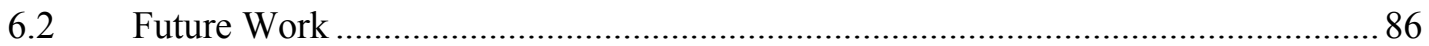

Appendix A Ethics Protocol Clearance ...................................................................... 87

Appendix B Research Ethics Protocol Application ............................................ 89

Appendix C Consent Form ............................................................................ 101

References............................................................................................................................. .104 


\section{List of Figures}

Figure 2.1 - The Hip Joint (Cross-sectional View) [27] ........................................... 8

Figure 2.2 - Anterior and Posterior View of the Femur [29] ...................................... 9

Figure 2.3 - Lateral View of the Pelvis [29] .......................................................... 9

Figure 2.4 - Anatomical Planes of Movement [26] ................................................... 10

Figure 2.5 - Hip Movements: Flexion, Extension, Abduction, Adduction, Internal

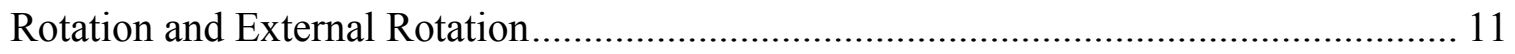

Figure 2.6 - Intra-cortical Pins into the Tibia, Femur, and Mid-patella with Triad Markers [37]

Figure 2.7 - An Ilizarov Circular External Fixator.................................................... 13

Figure 2.8 - Percutaneous Skeletal Tracker Consisting of the Four Halo Pins used to Fix the Device and the Four Retro-reflective Markers [40] ............................................ 14

Figure 3.1 - The Location of HJC Based on X-ray Imaging ..................................... 18

Figure 3.2 - Pelvic Anatomical Landmarks and Anatomical Coordinate System [9]..... 19

Figure 3.3 - The Position of Marker $j$ on the Surface of a Sphere with Centre $c$ and

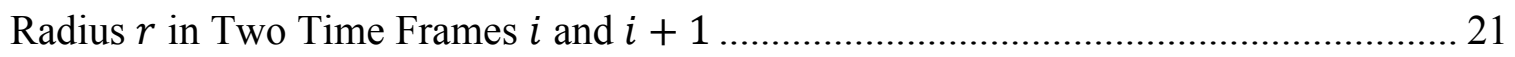

Figure 3.4 - Transformation of the Joint Centre between the Global and Local Coordinate

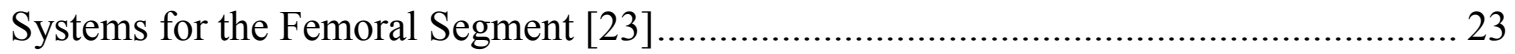

Figure 3.5 - The Marker Displacement in the Bone System during an Activity [16] ..... 27

Figure 4.1 - Overall Process for STA Assessment and Compensation .......................... 43

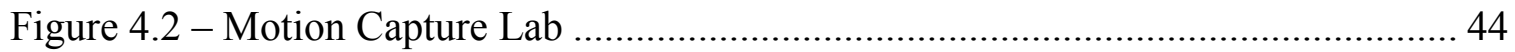


Figure 4.3 - Vicon Wall-mounted Near-infrared Camera ............................................... 44

Figure 4.4 - Calibration of the Vicon system: a) L-Frame b) Wand ................................ 46

Figure 4.5 - Skin Markers Configuration .................................................................. 47

Figure 4.6 - Subject Positions during Trial, Abduction , Flexion, and Extension ........... 48

Figure 4.7 - Ultrasound Imaging Machine ………………....................................... 50

Figure 4.8 - Ultrasound Transducer Probe ..................................................................... 51

Figure 4.9 - Passing a Plane through Each Curve ............................................................ 54

Figure 4.10 - Determining Points on the Bone.............................................................. 55

Figure 4.11 - Global and Local Coordinate Systems for the Femoral and Pelvic Segments 59

Figure 5.1-Subject's Thigh Markers Configuration ...................................................... 61

Figure 5.2 - Subject Positions during Optical Motion Capture, a) Standing, b) Abduction,

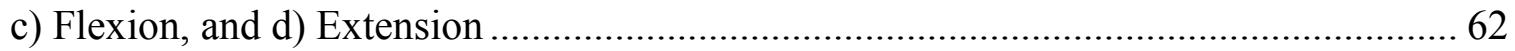

Figure 5.3 - 3D Reconstruction of Markers Positions of a Subject................................... 63

Figure 5.4 - Locations of Ultrasound Depth Measurements ........................................... 64

Figure 5.5 - Subject Positions during Ultrasound Depth Measurements, a) Standing, b)

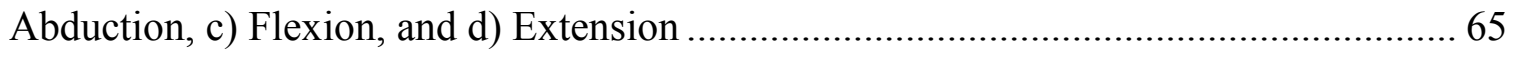

Figure 5.6 - Ultrasound Depth Measurement at a Marker Position, Standing Position ... 66 Figure 5.7 - Ultrasound Depth Measurement at a Marker Position, Flexion .................... 67 Figure 5.8 - Ultrasound Depth Measurement at a Marker Position, Extension ................ 67 Figure 5.9 - Ultrasound Depth Measurement at a Marker Position, Abduction .............. 68 Figure 5.10 - Curve Fitting to Motion Capture Data and Determination of Bone Positions at 3 Key Points Positions of Standing Position.................................................................. 71

Figure 5.11 - Transformation of Standing Markers to Flexion Movement....................... 72

Figure 5.12 - Transformation of Standing Markers to Extension Movement .................. 73 
Figure 5.13 - Transformation of Standing Markers to Abduction Movement 73

Figure 5.14 - Displacement of Markers (magnitude), Flexion...................................... 74

Figure 5.15 - Displacement of Markers (magnitude), Extension ................................... 74

Figure 5.16 - Displacement of Markers (magnitude), Abduction ................................ 75

Figure 5.17 - Box-plots of STA Components of the First Ring of Markers Configuration

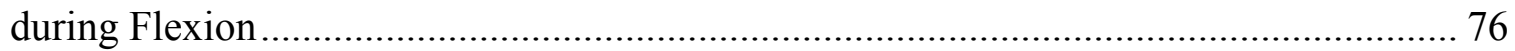

Figure 5.18 - Box-plots of STA Components of the Second Ring of Markers

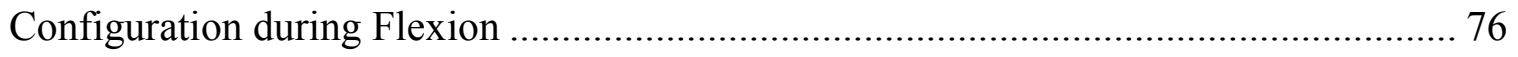

Figure 5.19 - Box-plots of STA Components of the Third Ring of Markers Configuration

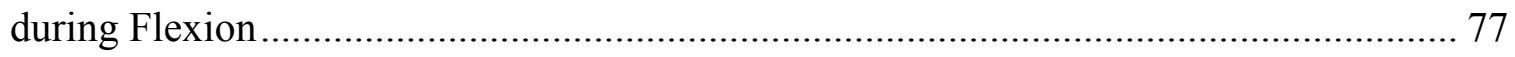

Figure 5.20 - Box-plots of STA Components of the Fourth Ring of Markers

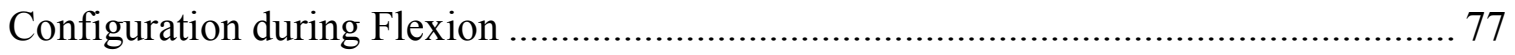

Figure 5.21 - Box-plots of STA Components of the First Ring of Markers Configuration

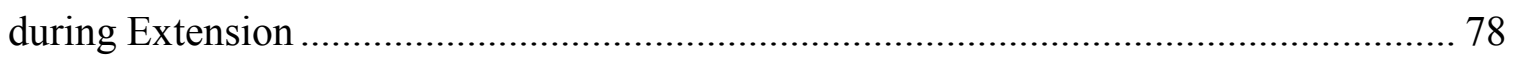

Figure 5.22 - Box-plots of STA Components of the Second Ring of Markers

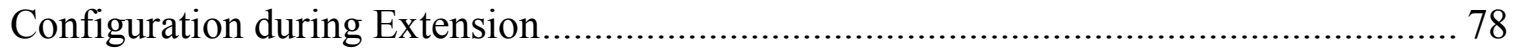

Figure 5.23 - Box-plots of STA Components of the Third Ring of Markers Configuration

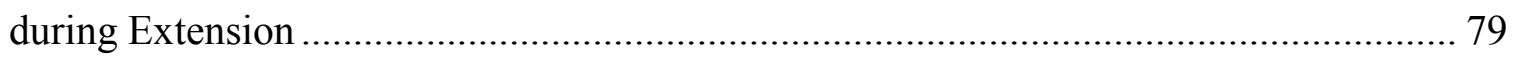

Figure 5.24 - Box-plots of STA Components of the Fourth Ring of Markers

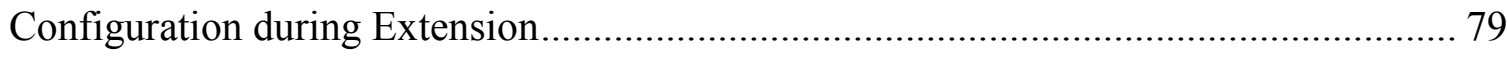

Figure 5.25 - Box-plots of STA Components of the First Ring of Markers Configuration

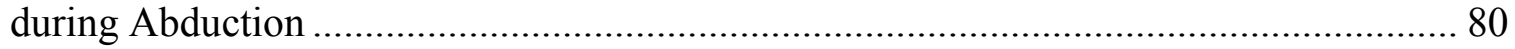

Figure 5.26 - Box-plots of STA Components of the Second Ring of Markers Configuration during Abduction.

Figure 5.27 - Box-plots of STA Components of the Third Ring of Markers Configuration during Abduction 81 
Figure 5.28 - Box-plots of STA Components of the Fourth Ring of Markers

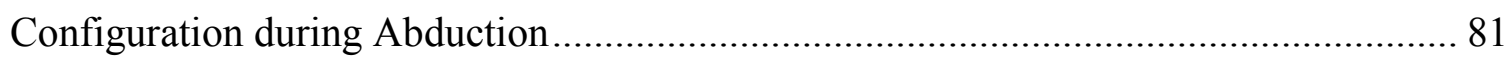

Figure 5.29 - Hip Joint Centre Location Error Using SCoRE Algorithm, Standing

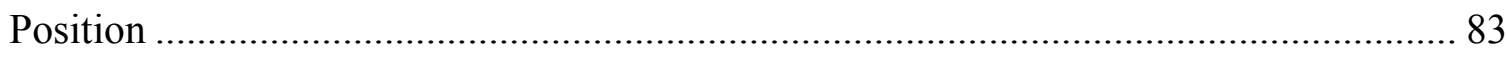

Figure 5.30 - Hip Joint Centre Location Error Using SCoRE Algorithm, Flexion.......... 83

Figure 5.31 - Hip Joint Centre Location Error Using SCoRE Algorithm, Extension...... 84

Figure 5.32 - Hip Joint Centre Location Error Using SCoRE Algorithm, Abduction ..... 84 


\section{List of Tables}

Table 1.1 - Subject's Age, Weight, Height, and Gender............................60

Table 1.2 - Ultrasound Depth Measurements for one of the Participants ..............69

Table 1.3 - Coefficient of Variation of Ultrasound Depth Measurements at each Marker

Location during all Movements of one Subject.................................... 70 


\section{Abbreviations}

ACS Anatomical Coordinate System

AL Anatomical Landmark

AP Anteroposterior

ASIS Anterior Superior Iliac Spine

CAST Calibrated Anatomical System Technique

CTT Centre Transformation Techniques

CoR Centre of Rotation

CV Coefficient of Variation

DoF Degrees of Freedom

FAI FemoroAcetabular Impingement

HJC Hip Joint Centre

MoCap Motion Capture

MRI Magnetic Resonance Imaging

OA Osteoarthritis

PSIS Posterior Superior Iliac Spine

PSM Pliant Surface Modeling

PST Percutaneous Skeletal tracker

PW Pelvic Width

RBM Rigid Body Modeling

RE Regression Equations

ROM Range Of Motion

SCoRE Symmetrical CoR Estimation

STA Soft Tissue Artefact

SVD Singular Value Decomposition

UDM Ultrasound Depth Measurement 


\section{Chapter 1: Introduction}

\subsection{Motivation}

The human hip joint is a ball-and-socket joint that connects the hip bone and femur. The accurate location of the Hip Joint Centre (HJC) is a necessary component in functional analysis of the hip to measure skeletal parameters and describe human motions. Centre of the hip joint is considered as a reference location in various medical applications:

- Many people have limited range of motion of hip due to hip joint disorders such as osteoarthritis (OA). A potential cause of osteoarthritis is Femoro-Acetabular Impingement (FAI), especially in young and active people [1]. Two types of FAI (i.e., abnormal contacts between femoral and acetabular surface) are cam and pincer impingement. In these two types, the head of the femur or the acetabular rim is deformed and the femoral head-neck starts to hit the acetabular over-coverage and destroys itself [2]. Determination of the functional centre of the hip and relative motions between the femur and acetabulum provide useful information to diagnosis and treat FAI.

- A treatment for patients who suffer from degenerative hip or knee joint is joint replacement procedure. Precise alignment of the leg is an important factor to have successful surgical results which lead to restoration of natural alignment of the bone, less need for revisions surgeries and quick recoveries with less pain. Centre of the hip joint is needed as a reference point to improve the accuracy of alignment of lower limbs in computer-assisted hip and knee surgeries [3]. 
- Determination the parameters of modeling muscle activity which is used to simulate movements and effects of musculoskeletal surgeries [4]. As some of these models are based on the bones geometries and joints kinematics, the accurate location of the centre of the hip joint is required.

The location of the hip joint can be estimated through various methods which can be divided into three categories: image-based techniques, predictive methods, and functional methods $[5,6]$. Imaged-based determination of the hip centre requires a medical imaging modality such as x-ray radiographs, CT scans and magnetic resonance imaging (MRI). In these techniques, standardized images of the pelvis are obtained and the HJC location is considered as the geometrical centre of the head of the femur modeled as a circle in 2D images and a sphere in 3D images. One error in determination of HJC location using image-based techniques is caused by the assumption of the femoral head as a sphere although it is not perfectly spherical. The use of image-based determination of the HJC is limited as MRI-based techniques require expensive medical imaging and the other modalities in this category expose the subject to ionizing radiation [7, 8]. Predictive methods estimate the HJC based on regression equations between palpable bony landmarks and the joint centre [8]. These methods need the exact locations of bony landmarks in the calculations of HJC. The accuracy depends on identification of the anatomical landmarks and the error range of them in able-bodied adults was reported to be $25-30 \mathrm{~mm}$ [9]. This error is higher in people with pelvic deformities due to the assumption of hip symmetry for both legs in these methods [10]. The error associated with the predictive methods has led to an increased interest in identifying hip joint centres using functional methods. Functional methods are based on the relative motion of the femur to the pelvis. In order to have the functional centre of the hip joint, the relative motion of the femur to the pelvis must be accurately measured. Optical motion capture systems are the most used systems for non-invasive study of human movement. In optical motion capture, reflective markers are attached to the skin and cameras track 3D trajectories of the markers. In this technique, the internal bone is inaccessible and markers are not rigidly placed on the bone; therefore, there is the relative motion between the markers and bone due to muscle activity and skin deformation, which is known as 
Soft Tissue Artefact (STA). One of the main objectives in human movement analysis is the assessment and correction of the soft tissue artefact, as it is the main source of error. Several techniques have been presented to assess STA, which are separated into five categories: intra-cortical pins, external fixators, percutaneous trackers, radiographic examinations, and magnetic resonance imaging [11]. Techniques based on intra-cortical pins, external fixators, and percutaneous trackers can represent relatively accurate measurements of bone motion; but the use of these techniques is limited as the procedures of applying them are invasive and subjects may experience pain. The main drawbacks of techniques based on radiographic examinations are these methods are invasive due to radiation exposure, the 3D measurements of the STA are estimated from two planes which provide 2D information, and these techniques require extensive processing of image data [12]. MRI-based techniques require expensive medical imaging and they are not suitable for everyday clinical measurements and analyses [13].

Several methods have been proposed to reduce the STA effects: the solidification model, multiple anatomical landmark calibration, pliant surface modelling, dynamic anatomical landmark calibration, point cluster technique, global minimization, and techniques based on MRI $[11,13]$. The solidification model does not compensate the STA effects well as it can only identify erroneous frames $[11,14]$. Dynamic calibration and multiple anatomical landmark calibration are based on invalid assumptions (linearity assumptions) and they are time consuming because they require additional data acquisitions [15]. The limitations of the point cluster technique are an overabundance of markers and instability $[16,17]$. The drawback of the global optimization technique is that it simplifies joints structures that are not subject-specific, which cannot be applied to people with hip joint disorders $[18,19]$. MRI-based techniques are expensive and consequently they are inappropriate for everyday clinical uses.

Despite the numerous methods proposed, the objective of a reliable non-invasive and clinical assessment and correction of STA in human hip joint kinematics is still being investigated, and this is the domain where our work lies. 


\subsection{Objectives and Contributions}

The main objectives of this study are as follows:

- Developing a non-invasive experimental setup to measure marker trajectories and displacements during the lower limb motion to determine of the functional hip joint centre.

- Proposing a non-invasive method to quantify and assess the pattern of soft tissue artefact in human hip joint kinematics.

- $\quad$ Proposing a method to improve the functional centre of the hip joint.

To pursue our objectives, our research has three parts: optical motion capture experiment, ultrasound depth measurements (UDM), and data analysis. As existing methods to assess and reduce STA effects are mainly invasive and inappropriate for clinical daily uses, our focus is to quantify and compensate STA non-invasively. To this end, our method consists of two experimental parts: motion capture analysis and ultrasound measurements. Motion capture analysis is performed through an optical motion capture system that is the most common system in human movement studies since it is noninvasive. In this experimental part, we define a proper configuration of the markers to place them on the subject's lower limbs. Optical motion capture system tracks trajectories of the markers to have realistic motions of the lower limb for a range of motions of the hip joint (standing, flexion, extension, and abduction). To quantify and compensate for STA, our approach is to combine motion capture data and the information from changes in the muscle thickness at the marker positions. We use ultrasound imaging to measure the changes in tissue thickness at the marker positions for the same standing and extended positions as motion capture analysis. The use of ultrasound imaging for the functional determination of the HJC was introduced by Sangeux et al. [20] and using it, in combination with motion capture as a validation tool, was introduced by Peters et al. [21]; however Upadhyaya et al. [22] first introduced the idea of combining both ultrasound and Motion Capture into a single analysis process; we have continued this trend of combining ultrasound information with motion capture. Ultrasound is one of the preferred imaging modalities because this modality is non-invasive and poses no harm to human bodies and, in addition, it is a relatively low cost and portable imaging modality. 
Using ultrasound imaging to measure tissue thickness from the bone position, ultrasound echoes are reflected strongly from the bone surface and cause high intensity pixels in the image representing the bone surface.

Next step after experimental parts is data analysis. In this step, we propose a mathematical approach to determine the bone positions of three markers which have least changes in their tissue thickness and use these positions on the bone to quantify STA. Our last aim is to calculate the HJC using a coordinate transformation technique, symmetrical centre of rotation estimation (SCoRE) algorithm [23]. In order to have an accurate HJC location, we use the displacement of the markers from the previous step and eliminate STA effects from the marker's data used in the SCoRE algorithm.

\subsection{Publications}

The achievements of our work have been detailed in three conferences articles. The complete list of publications made during this thesis work is as follows:

A. Rouhandeh, C. Joslin, Z. Qu, and Y. Ono, "Non-invasive Assessment of Soft-tissue Artefacts in Hip Joint Kinematics Using Motion Capture Data and Ultrasound Depth Measurements", The 36th Annual International Conference of the IEEE Engineering in Medicine and Biology Society (EMBC), Chicago, 2014.

A. Rouhandeh, C. Joslin, Z. Qu, and Y. Ono, "Soft-tissue Artefact Assessment and Compensation in Hip Joint Kinematics Using Motion Capture Data and Ultrasound Depth Measurements", International Conference on Biomedical Engineering and Systems (ICBES), Prague, 2014.

A. Rouhandeh, C. Joslin, Z. Qu, and Y. Ono, "Quantification of Soft Tissue Artefacts Using Motion Capture Data and Ultrasound Depth Measurements", International Conference on Bioinformatics and Biomedical Engineering (ICBBE), Toronto, 2014.

* Selected to publish in International Journal of Medical, Health, Pharmaceutical and Biomedical Engineering, vol. 8, no. 6, pp. 29-33, 2014.

\subsection{Outline}

The remainder of this thesis is organized as follows: Chapter 2 provides an overview of the hip joint anatomy, clinical movements of the hip joint and the existing instrumentations and techniques to record human $3 \mathrm{D}$ motion for motion analysis. Chapter 3 is dedicated to 
describe all existing methods for determination of the HJC location. A complete survey of the methods to minimize and compensate the STA effects in motion analysis is given. The three remaining chapters describe our solution on the topic of STA assessment and correction in hip joint kinematics: in Chapter 4, we propose a non-invasive method to quantify and minimize STA in determination of the HJC using SCoRE algorithm. Our solution is based on the information from ultrasound depth measurements and optical motion capture analysis. The details of the experimental procedures and data analysis are provided. Experiments are carried out for ten subjects. The steps of the subjects' experiments and corresponding results are explained in Chapter 5. Finally, we conclude with our achievements and future works in Chapter 6. 


\section{Chapter 2: Introduction to the Hip Joint}

\subsection{Introduction}

Human motion analysis attempts to obtain quantitative information about the patterns of the musculoskeletal functions and movements. Human motion analysis has a wide range of applications in computer vision, sports for analysis of athletic performance, biology, medicine such as for tracking the movements and behaviors of joints and their diagnosis and surgical treatment, physical rehabilitation, designing prosthetic devices, and humancomputer interaction [24]. So there is a great need for quantitative measurements of human movement. Estimation of hip joint kinematics has received increasing attention since many applications, such as preoperative planning simulation, human gait analysis, and hip joint disorders, require the hip joint performance. In clinical applications, motion measurements can be used to compare the function of normal and abnormal hip joints. To accurately simulate the hip joint, we need to estimate the Centre of Rotation (CoR) of the hip accurately.

To this aim, this chapter provides an overview of hip joint anatomy. Then, the various

clinical movements of the hip joint are expressed. Eventually, we present the existing instrumentations and techniques to record human 3D motion to use in motion analysis studies. 


\subsection{Hip Joint Anatomy}

Hip joint is one of the most important joints of human body and is a major weightbearing joint. The hip joint is a ball-and-socket type joint that connects hip bone and the femur [25]. This joint allows us to walk, run and jump with large range of hip motion in several directions; it is embedded within the thigh muscles and provides body locomotion and movement with these muscles [26]. Figure 2.1depicts a cut-section view of hip joint.

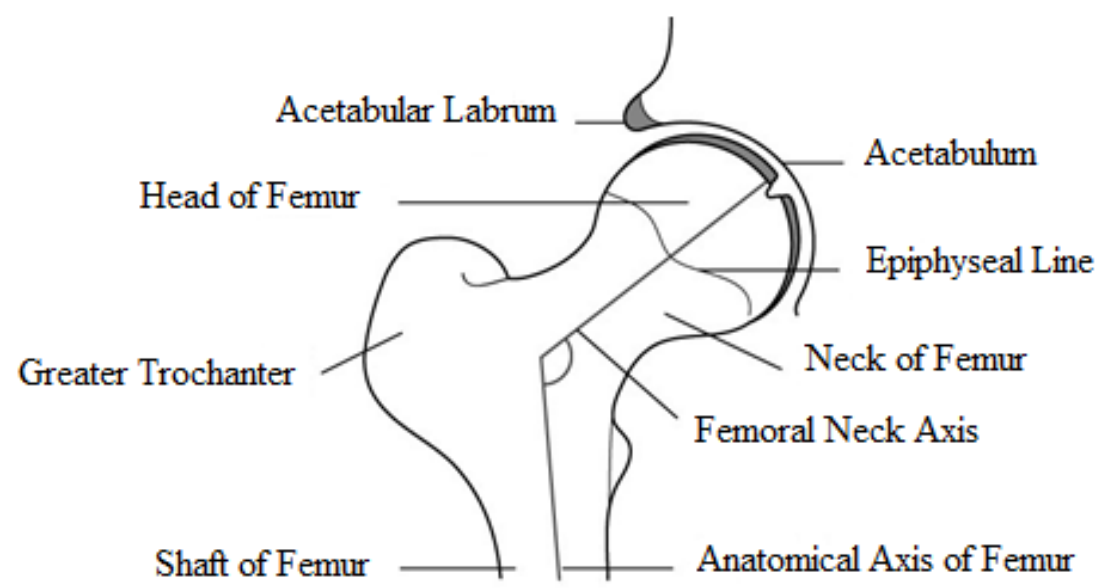

Figure 2.1 - The Hip Joint (Cross-sectional View) [27]

The hip joint consists of femur and pelvis bones. The femoral head (ball) fits into a deep socket in the pelvis bone, the acetabulum (socket) [28]. The femur, or thigh bone, is the longest and strongest bone in the body.

As it is shown in Figure 2.2, the proximal end of the femur is composed of a head, a neck, greater trochanter, and lesser trochanter. The almost spherical femoral head fits into the acetabulum and is attached to the shaft by the femoral neck. The neck of the femur provides the hip joint wide range of motion. The angular value between the neck and the femoral body varies throughout life; it is larger at birth and decreases with age [26]. The hip bone or pelvis is a flat and irregularly shaped bone. Each hip bone consists of three bones, ilium, ischium and pubis, which meet at the acetabulum [26]. The bones are illustrated in Figure 2.3. These three bones are separate bones from each other at birth but become fused to form one bone in the adult [26]. 


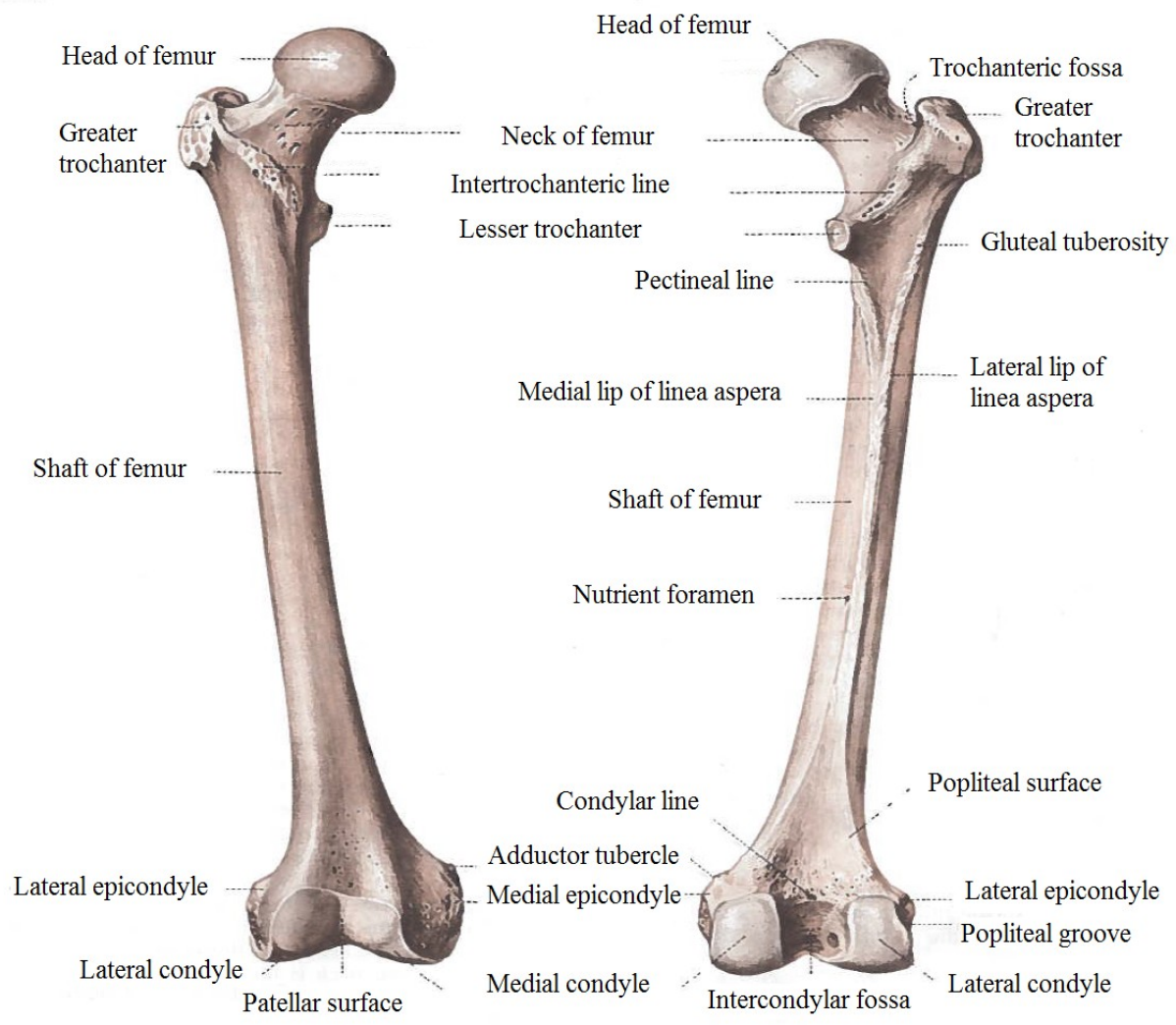

Figure 2.2 - Anterior and Posterior View of the Femur [29]

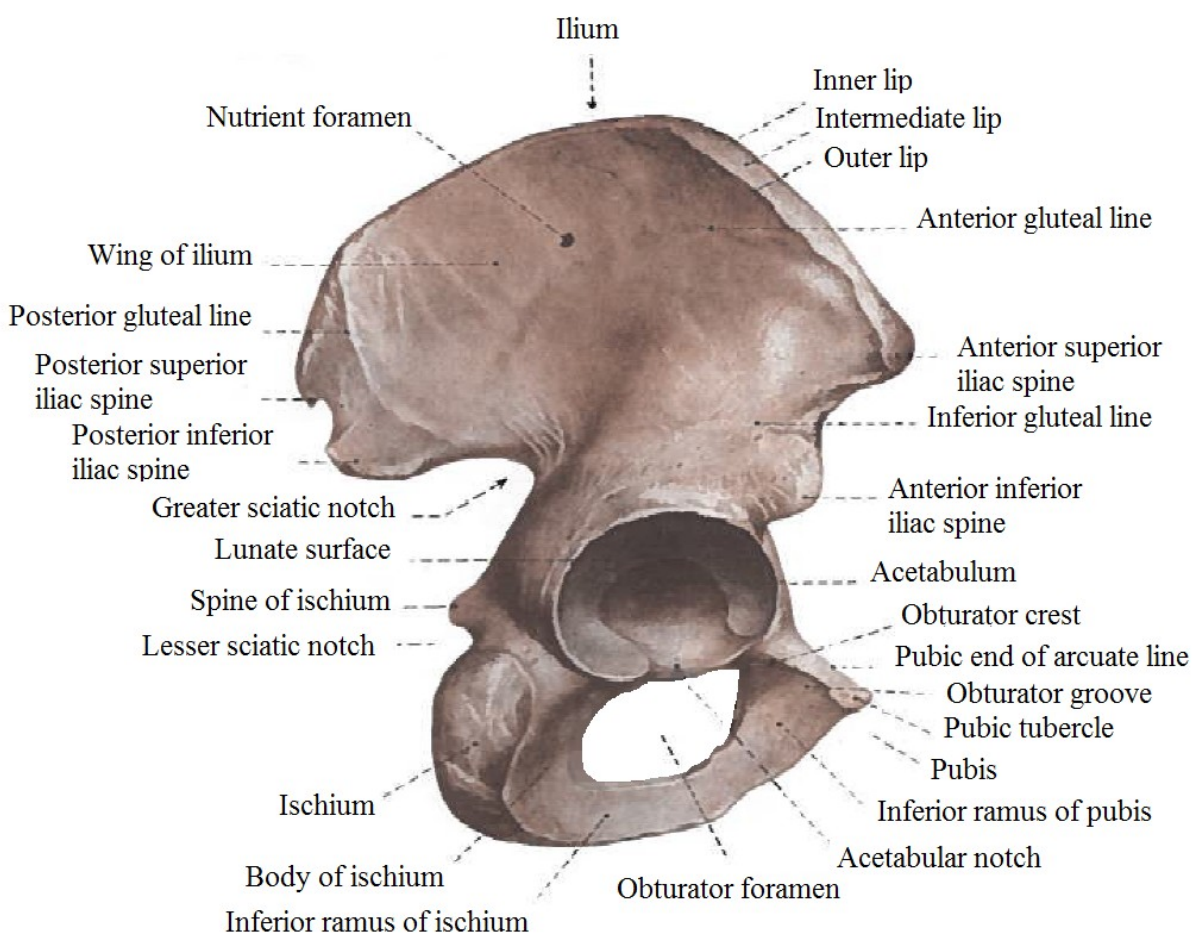

Inferior ramus of ischium

Figure 2.3 - Lateral View of the Pelvis [29] 


\subsection{Clinical Movements of the Hip Joint}

This section aims at understanding the clinical movements of the hip joint as the hip joint flexibility is defined by its Range of Motion (RoM), and RoM is a basic parameter for diagnosing hip diseases [30, 31]. Anatomical planes are illustrated in Figure 2.5. In a clinical environment, hip joint motions can be measured with goniometer or inclinometer [32]. Most standard goniometers have a protractor with two hinged arms [33]. The protractor is in the centre of goniometers and measures angles between two arms. An inclinometer is a modified digital protractor which uses gravity to indicate the range of motion in the frontal and sagittal planes. Using a two-arm goniometer is the most common tool for to measuring hip joint motion but inclinometers are more reliable for measuring the rotation of the hip [32].

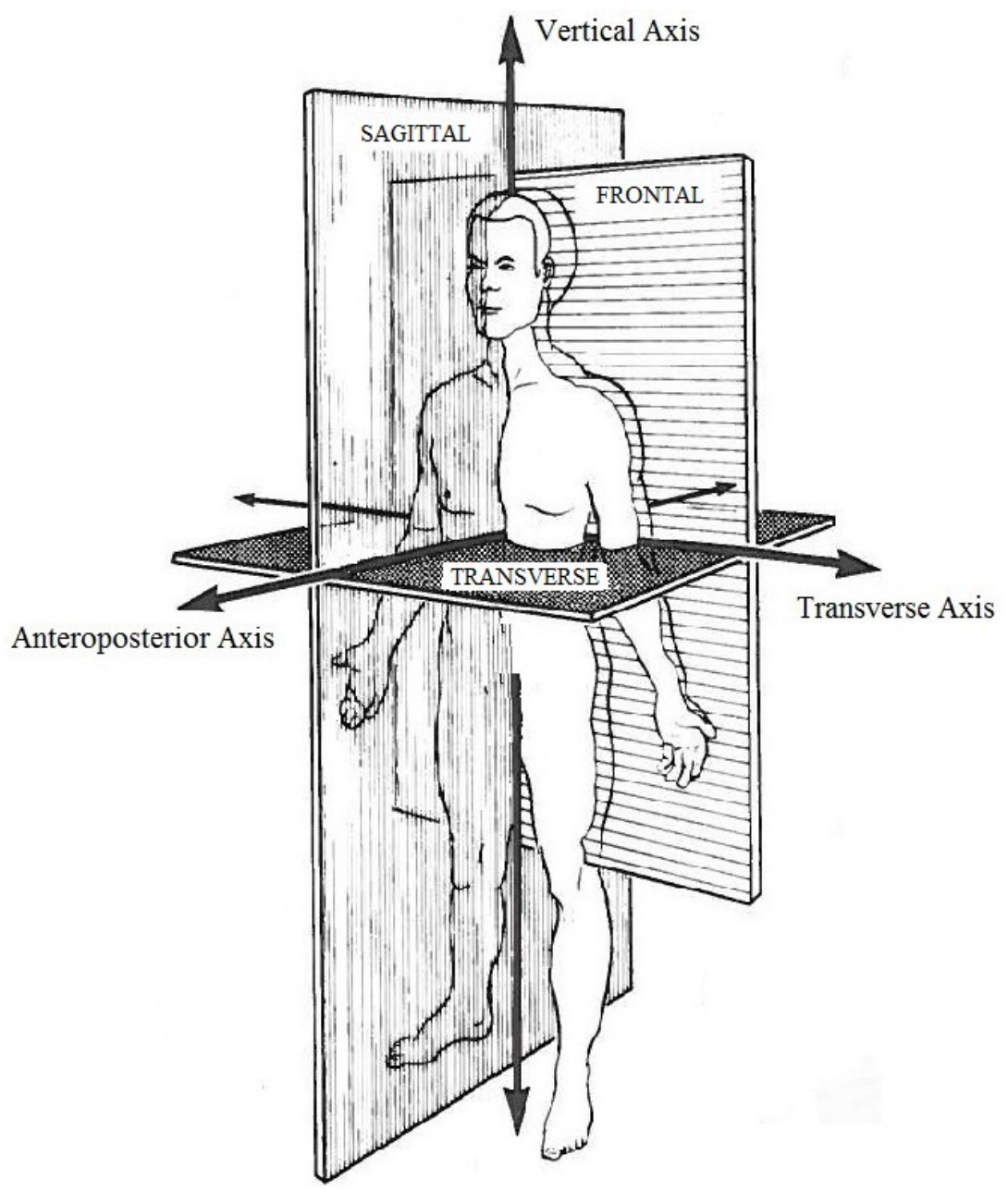

Figure 2.4 - Anatomical Planes of Movement [26] 
The hip joint movements in the sagittal plane are flexion and extension, in the frontal plane are abduction and adduction and in the transverse plane are internal and external rotation. Figure 2.6 depicts these movements.
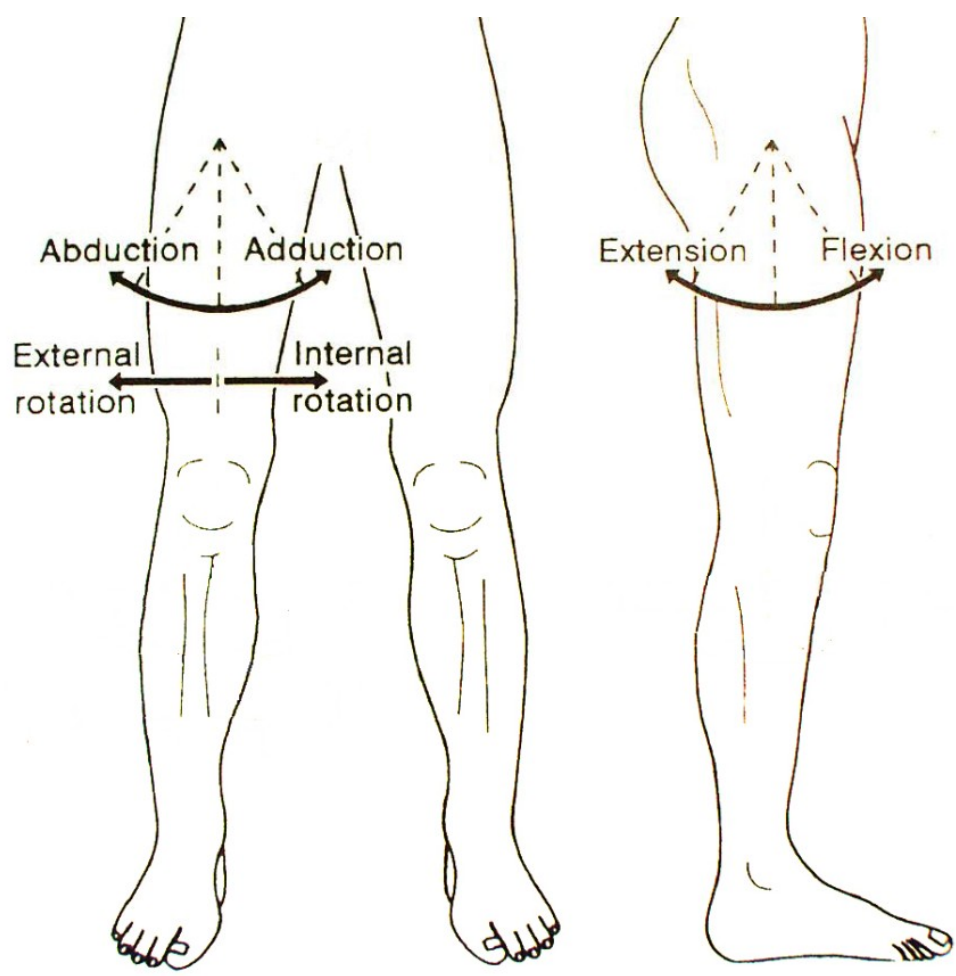

Figure 2.5 - Hip Movements: Flexion, Extension, Abduction, Adduction, Internal Rotation and External

\section{Rotation}

Flexion moves the femur forward to decrease the angle at the joint. The range of hip joint flexion extremely depends on the knee; when the knee is extended the hip flexion range can reach 90 degrees but this range can reach 150 degrees when the knee is flexed. Extension moves the femur backward. The range of extension varies from 0 to 35 degrees, which is smaller than flexion range. Hip abduction is movement of femur outward to the side, away from the other femur. The range of abduction is approximately 55 degrees. Hip adduction moves the femur toward the midline of the body and its range can reach 45 degrees. Internal rotation moves the femur around the vertical axis, toward the midline, with a range of approximately 50 degrees. External rotation moves the femur around its vertical axis away from the midline, with a range of approximately 55 degrees [34]. 
Hip joint motion range varies according to several factors. Hip joint motion significantly decreases with age and hip joint diseases such as femoroacetabular impingement.

\subsection{Techniques for Human 3D Motion Recording}

In order to obtain the CoR we need to determine the motion of the femoral head relative to the hip joint. Therefore, we need to use a technique to record human movement. Instrumentation and techniques for human movement recording can be divided into two groups: bone-based and skin-based. In bone-based techniques, human kinematic data is collected from pins attached to the bones or determining bones positions by medical imaging. Skin-based techniques measure human kinematic data by tracking markers and devices attached to the skin surface. The techniques are described in detail in the following subsections.

\subsubsection{Bone-based Techniques}

Bone-based techniques can be distinguished into four categories: intra-cortical bone pins, external fixation devices, percutaneous trackers, and medical imaging techniques [35].

\section{- Intra-cortical Bone Pins}

There are numerous studies that used intra-cortical pins to analyze human 3D kinematics during a variety of activities. In these studies, intra-cortical bone pins have been used to measure skeletal motion directly by attaching markers to the pins inserted into the bones. Then, the position, velocity and acceleration of the bones are determined by tracking the trajectories of the markers [36, 37]. While intra-cortical bone pin techniques provide an accurate representation of skeletal motion, it is not suitable for everyday clinical measurement since the procedure is invasive and the subject may experience pain [38]. Figure 2.8 shows an example of the insertion of intra-cortical bone pins into the tibia, femur, and mid-patella. 


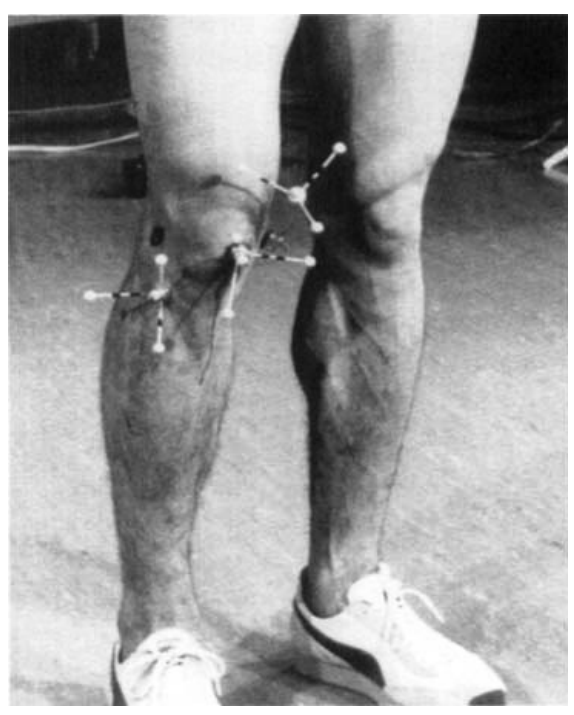

Figure 2.6 - Intra-cortical Pins into the Tibia, Femur, and Mid-patella with Triad Markers [37]

\section{- External Fixation Devices}

External fixation devices have been used in some experiments to obtain accurate kinematic data during gait [39]. The components of external fixators are wires, pins and rods fixed to the bone (as shown in Figure 2.9). Motion data of the segment of interest is calculated from markers attached to the external fixator.

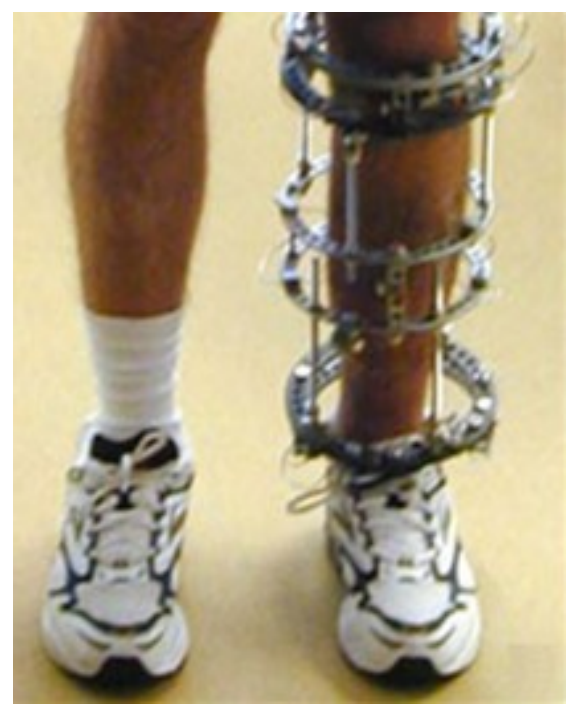

Figure 2.7 - An Ilizarov Circular External Fixator

The use of these devices is limited because they are invasive and cannot be used for studying the behavior of two bones at the same time. 


\section{- Percutaneous Skeletal Trackers}

Percutaneous skeletal trackers (PST) are devices that attach markers to a skeletal segment by using a number of halo pins inserted into the opposite sides of the segment (as shown in Figure 2.10) [40].
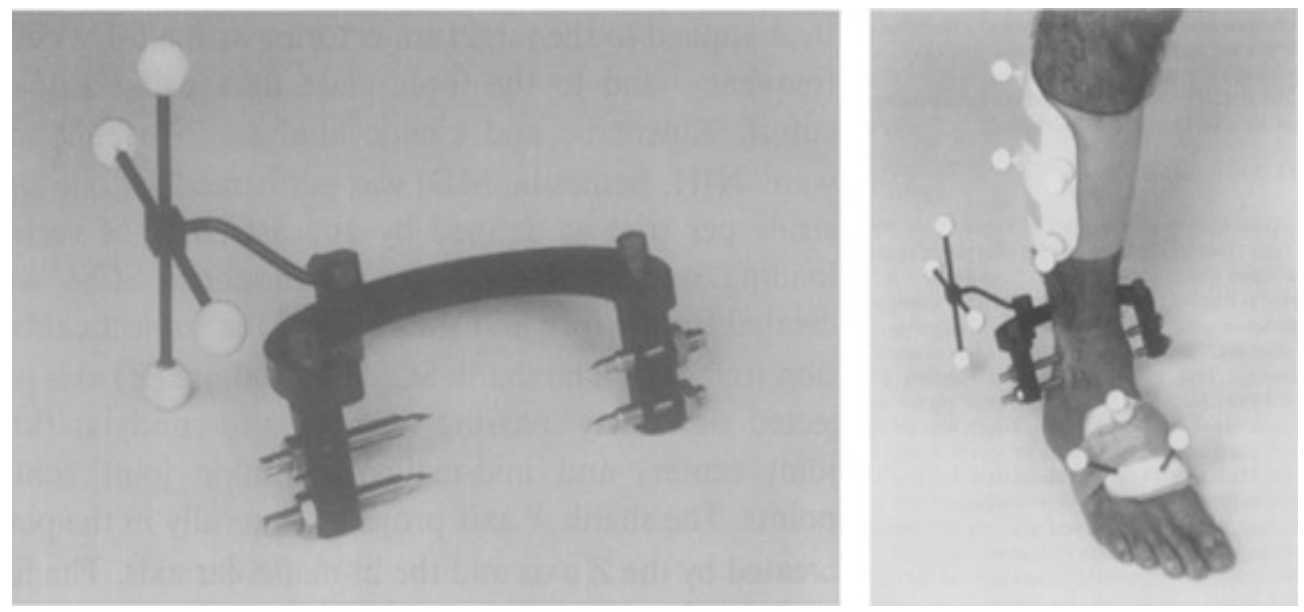

Figure 2.8 - Percutaneous Skeletal Tracker Consisting of the Four Halo Pins used to Fix the Device and the Four Retro-reflective Markers [40]

The limitations of these devices are that the procedure of applying them is invasive and these devices restrict joint motion during the activity.

\section{- Medical Imaging Techniques}

Medical imaging modalities for the study of kinematics of the human skeletal system are Roentgen stereophotogrammetry and fluoroscopy. Roentgen stereophotogrammetry uses two simultaneous radiographs to obtain two projections of an area of interest [41]. In order to have an accurate estimation of bone motion, metallic markers are implanted in the bone [42]. Fluoroscopy is an imaging technique that provides real-time and moving images of the position and orientation of body segment through the use of an x-ray source [43]. The drawback of medical imaging techniques is the radiation exposure.

Since the bone-based techniques are invasive, including pins insertion into the bone or exposing the subject to radiation, skin-based techniques can be considered for everyday kinematic gait analysis. 


\subsubsection{Skin-based Techniques}

The most common used techniques to collect motion data are skin-based techniques. Skin-based techniques are noninvasive and can be categorized into three categories: motion capture systems, video-based systems, and accelerometers and gyroscopes.

\subsubsection{Motion Capture}

Motion capture (MoCap) systems involve the recording of reflective markers or small devices attached to the skin surface. Three types of motion capture systems are mechanical, magnetic, and optical [44].

\section{- Mechanical Systems}

Mechanical systems use angle sensors to measure joint angles. A set of sensors is attached to the body as an exoskeleton motion capture system. During the activities, the exoskeleton moves and sensors placed on the joints measure the rotations. This system is not accurate since it determines the positions from the rotations.

\section{- Magnetic Systems}

This system uses magnetic sensors attached to the body to measure the magnetic field of a transmitter source. The magnetic system is real time and sensors are never occluded. The drawback of this system is that the presence of metals in the experimental environment can disturb the measurements.

\section{- Optical Systems}

Optical motion capture system consists of cameras to track reflector markers placed on the body. Optical motion capture systems are the most used systems in the study of human movement. However, these systems are expensive and the markers are sometimes obscured from vision of less than two cameras which results in inaccurate measurements of human movement [45]. 


\subsubsection{Video-based Systems}

Video-based systems capture human motion as video data from multiple cameras and use computer vision methods to reconstruct human motion in three dimensions [46]. The idea behind markerless motion capture systems is that the accurate techniques to capture human movements require the attachment of markers on the body which affect the natural movements [47]. These systems can be divided into two categories: active and passive vision systems. Active systems consists of a light source and sensors providing accurate measurements of human motion while passive systems are only based on capturing images [47]. Video-based systems are less accurate and cheaper compared to motion capture systems.

\subsubsection{Accelerometers and Gyroscopes}

Another technique to obtain kinematics data (such as shank and thigh inclination angles, and knee angle) uses body-fixed sensory devices, accelerometers and gyroscopes [48]. A gyroscope determines orientation based on the earth's gravity while an accelerometer measures non-gravitational acceleration. Several studies used accelerometers and gyroscopes to measure human joint angle. Willemsen et al. used four accelerometers on each segment to measure flexion-extension [49]. Heyn et al. used eight accelerometers and two gyroscopes to measure shank and thigh inclination angles [50]. Accelerometers and gyroscopes are portable and inexpensive compared to optical motion capture systems but they have worse accuracy and only provide relative angles, and cannot provide accurate distance measurements. 


\section{Chapter 3: Related Studies}

\subsection{Hip Joint Centre}

Accurate hip joint centre location is a necessary component in biomechanical human motion analysis to measure skeletal parameters and describe human motions [51]. In general, HJC determination is more difficult than the other human joints because this joint is far from palpable bony landmarks [52]. A variety of approaches have been proposed to estimate HJC location that can be divided into three categories: image-based techniques, predictive methods, and functional methods [5, 6]. Imaged-based techniques require a medical imaging modality to estimate the HJC location as the geometrical centre of the femoral head. Predictive methods estimate the HJC location relative to palpable bony landmarks. Functional methods determine the HJC from the relative motion of femur and pelvis. Since functional methods consider hip ranges of motion, they have been reported to be more accurate for functional analysis of the hip and human motion analysis [53]. HJC determination by functional methods suffers inaccuracy due to soft tissue artefact, which is discussed later in this chapter. We first describe all methods for determining the HJC location. Then, we present an overview of previous studies which were conducted to assess STA and compensate for its effects. 


\subsubsection{Image-based Techniques}

Determination of hip joint centre can be performed by certain medical imaging modalities such as X-ray radiographs, CT scans and MRI [8, 54, 55]. Standardized images of the pelvis are obtained and the HJC location is considered as the geometrical centre of the head of the femur modeled as a circle in $2 \mathrm{D}$ images and a sphere in $3 \mathrm{D}$ images. Figure 3.1 shows a standardized anterior-posterior radiograph of pelvis in which the HJC coincides with the geometrical centre of femoral head.

There is an inherent error in determination of HJC location using image-based techniques as they consider the femoral head as a sphere although it is not perfectly spherical. The use of image-based determination of the HJC is limited as MRI-based techniques require expensive medical imaging and the other modalities in this category expose the subject to ionizing radiation $[7,8]$.

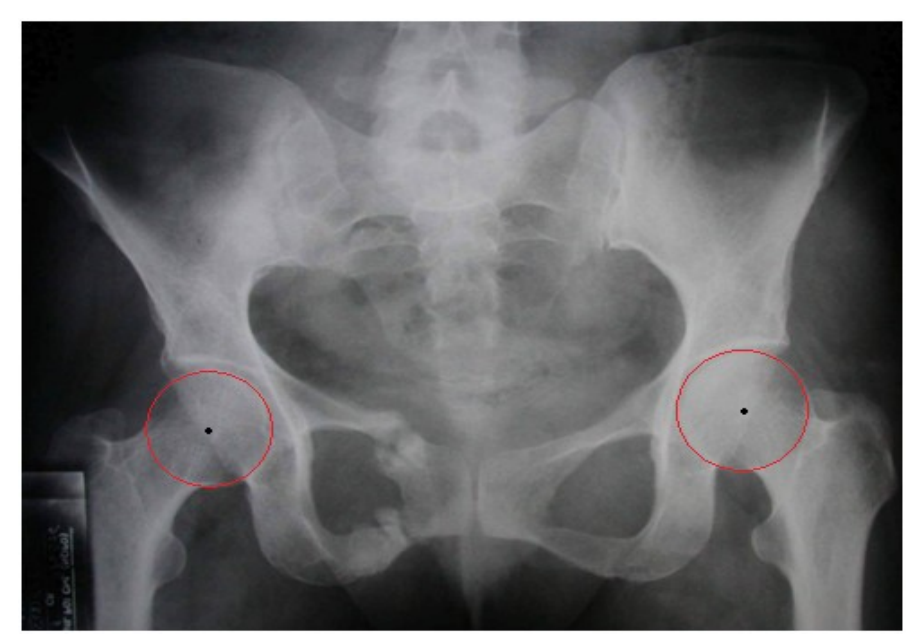

Figure 3.1 - The Location of HJC Based on X-ray Imaging

\subsubsection{Predictive Methods}

Predictive methods estimate the hip joint centre based on regression equations (RE) between palpable bony landmarks and the joint centre [8]. Two studies by Tylkowski's group and Andriacchi's introduced predictive methods [56, 57]. Tylkowski et al. proposed the HJC location as a constant percentage of the distance between the left and right anterior superior iliac spines (ASIS). They reported the percentages to determine the 
HJC location as $11 \%$ of the inter-ASISs distance medial, $12 \%$ distal, and $21 \%$ posterior to the ASIS. The HJC location by their method can be expressed as the Equation (1).

$$
x=-0.21 \mathrm{PW}, \quad y=-0.12 \mathrm{PW}, \quad z=-0.11 \mathrm{PW}
$$

Where $x, y$, and $z$ are the local coordinates of the HJC in the pelvic anatomical coordinate system (ACS) and PW is the distance between left and right ASIS. Figure 3.1 shows pelvic anatomical landmarks (AL) and a coordinate system with the ACS origin at the midpoint between the right and left ASIS. The z-axis is the line passing through both ASISs with positive direction from left-ASIS to right-ASIS. The $\mathrm{x}$-axis lies in the plane which is defined by the left and right ASISs and the midpoint between the left and right posterior superior iliac spines (PSIS) and with its positive direction forwards. The y-axis is specified by the cross product of $\mathrm{z}$-axis and $\mathrm{x}$-axis [58].

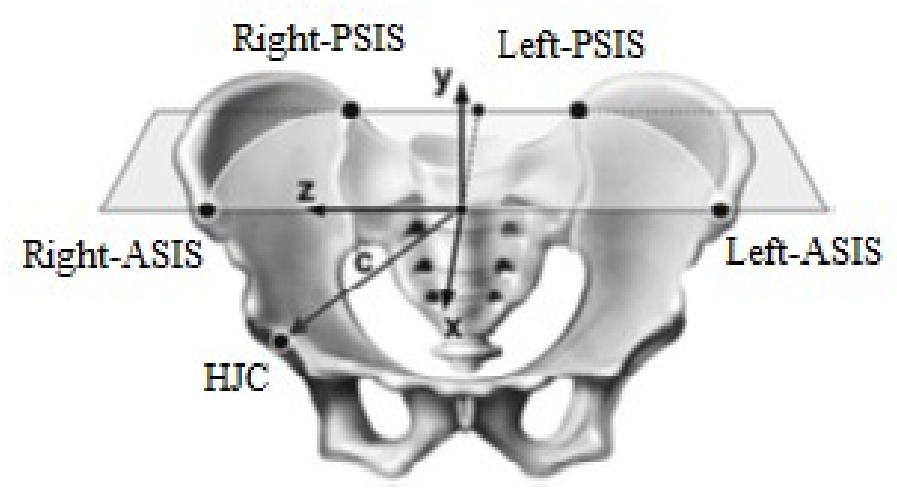

Figure 3.2 - Pelvic Anatomical Landmarks and Anatomical Coordinate System [9]

Tylkowski et al. examined 200 children's anterior-posterior (AP) and lateral pelvic xrays, but provided few details [56].

Andriacchi et al. determined the HJC location from the midpoint of the line between the ASIS and pubic symphysis, $1.5 \mathrm{~cm}$ or 1.5 to $2 \mathrm{~cm}$ distal to the midpoint of the line, in the frontal plane $[57,59]$. There are two more predictive methods which proposed later by Bell et al. and Davis et al. [60, 61]; The regression equations from their approaches are as Equations (2) and (3), respectively. 


$$
\begin{gathered}
x=-0.19 \mathrm{PW}, \quad y=-0.30 \mathrm{PW}, \quad z=-0.36 \mathrm{PW} \\
x=-0.95 \mathrm{D}+0.31 \mathrm{~L}-4, \\
y=-0.31 \mathrm{D}-0.96 \mathrm{~L}+13, \\
z=0.5 \mathrm{PW}-0.055 \mathrm{~L}+7
\end{gathered}
$$

Where $x, y$, and $z$ are the local coordinates of the HJC in the pelvic ACS, PW is pelvic width, $\mathrm{D}$ is anterior-posterior component of the distance between a point approximating the hip centre and the homolateral ASIS, and L is distance between ASIS and homolateral medial malleolus.

Predictive methods need the exact location of bony landmarks to calculate the HJC. The accuracy of these methods depends on identification of the anatomical landmarks, and the

error range of them in able-bodied adults was reported to be between $25-30 \mathrm{~mm}$ [9]. This error is higher in people with pelvic deformities due to the assumption of hip symmetry for both legs in these methods [10].The error associated with predictive methods has led to an increased interest in identifying hip joint centres using the functional methods.

\subsubsection{Functional Methods}

Functional methods determine the hip joint centre from the relative motion of the femur and pelvis. To determine the HJC using functional methods, three non-collinear markers are sufficient to define a segment's position and orientation in three dimensional. Functional methods are divided into two categories: sphere fitting and coordinate transformation methods. In the following subsections, each of the methods is explained in detail.

\subsubsection{Sphere Fitting}

The hip joint is generally considered as a ball-and-socket joint with three degrees of freedom (DoF). Ideally, if the pelvis is considered stationary, the markers placed on the femoral segment move on the surface of spheres with the hip joint centre being at the centre of them. Sphere fitting methods attempt to fit the movement of each marker on the thigh to the surface of a sphere, with the hip joint centre considered as its centre $[62,60$, 
63]. If the global positions of $m$ markers, $j=1,2, \ldots, m$, are given by $v_{i j}$ in the $\mathrm{n}$ time frames, $i=1,2, \ldots, n$, then the hip joint centre is obtained by minimizing the squared Euclidean distances between the marker positions and the sphere for all frames. Equation (4) expresses this analytical approach that is called the geometric sphere fit method [23, 64].

$$
f_{\text {Geometric }}\left(c, r_{1}, \ldots r_{m}\right)=\sum_{j=1}^{m} \sum_{i=1}^{n}\left(\left\|v_{i j}-c\right\|-r_{j}\right)^{2}
$$

Where $v_{i j}$ is the global position of marker $j$ in the time frame $i, c$ is the hip joint centre, and $r_{j}$ is the sphere's radius during the movement of marker $j$. Figure 3.2 illustrates the terms used in the sphere fitting methods. As shown in Figure 3.2, $r_{j}$ can be calculated as the average of Euclidean distances between the marker $j$ positions and the sphere centre in $n$ time frames.

$$
r_{j}=\frac{1}{n} \sum_{i=1}^{n}\left\|v_{i j}-c\right\|
$$

Where $n$ is the number of all frames of the movements of the femoral marker $j$.

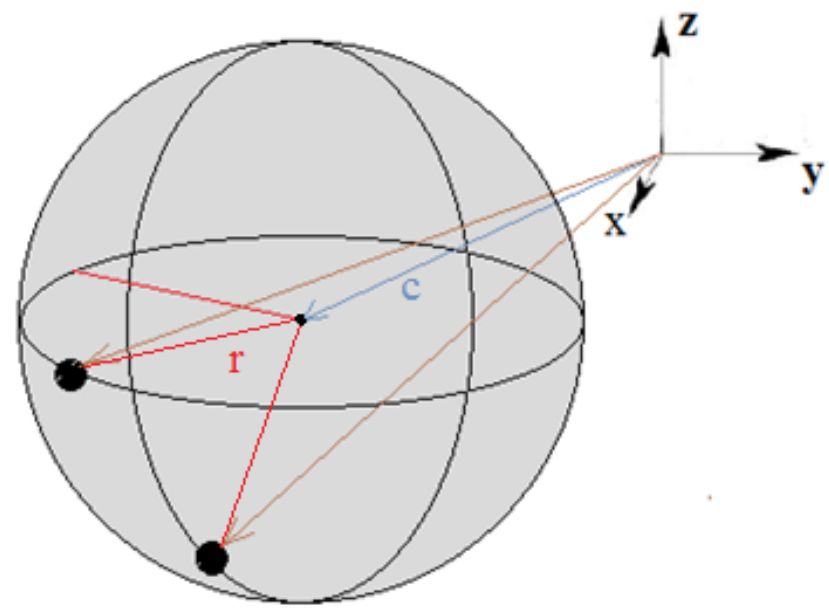

Figure 3.3 - The Position of Marker $\boldsymbol{j}$ on the Surface of a Sphere with Centre $\boldsymbol{c}$ and Radius $\boldsymbol{r}$ in Two Time Frames $\boldsymbol{i}$ and $\boldsymbol{i}+\mathbf{1}$ 
Since the Equation (4) is a nonlinear optimization problem, it doesn't have a closed form solution. This minimization problem can be solved iteratively $[65,66]$; which requires an initial estimation value for $c$. Another approach is the algebraic sphere fit, which uses a modified cost function minimization and has a closed solution $[67,68]$. This least square criterion is expressed in Equation (6).

$$
f_{\text {Algebraic }}\left(c, r_{1}, \ldots r_{m}\right)=\sum_{j=1}^{m} \sum_{i=1}^{n}\left(\left\|v_{i j}-c\right\|^{2}-r_{j}^{2}\right)^{2}
$$

The closed solution of Equation (6) is biased and does not compute the accurate centre of rotation [23]. A method which is known as the bias compensated algebraic sphere fit method has been proposed by Halvorsen, where the bias is iteratively reduced [69]. Another modified least squares criterion sphere fit method has been proposed by Pratt and is expressed in Equation (7) [70].

$$
f_{\text {Pratt }}\left(c, r_{1}, \ldots r_{m}\right)=\sum_{j=1}^{m} \frac{1}{r_{j}^{2}} \sum_{i=1}^{n}\left(\left\|v_{i j}-c\right\|^{2}-r_{j}^{2}\right)^{2}
$$

By this cost function, the bias in the algebraic sphere fit method is removed.

\subsubsection{Coordinate Transformation Techniques}

Coordinate transformation techniques assume that the distance between the markers attached to the femur and the pelvis are invariant during the movements. In these techniques a local coordinate system for each moving segment of the joint is defined, and then these local systems for all time frames are transferred into a global reference system to estimate the HJC. Coordinate transformation techniques are divided into two groups: one sided and two sided. One sided techniques consider one segment (femur) to determine the HJC and two sided techniques consider two segments (pelvis and femur). In the following, one of the most used one sided approaches, centre transformation technique (CTT), and one of the most accurate two sided approaches, symmetrical CoR estimation (SCoRE), are explained in detail [23]. 


\section{- Centre Transformation Technique}

Centre transformation technique assumes the pelvis and the CoR are stationary. At each time frame, a local coordinate system for the femoral segment is defined by at least three markers attached to the thigh. By having a local coordinate system, it is possible to define a rotation matrix $\boldsymbol{R}_{i}$ and translation vector $t_{i}$ of the femur at each time frame $i$. As the femur rotates around the CoR, which is assumed stationary, a fixed point $\tilde{c}$ (centre of rotation in every local coordinate system) is mapped to a corresponding point, $c$, in the global coordinate system, which is given by Equation (8).

$$
c=\boldsymbol{R}_{i} \tilde{c}+t_{i}, \quad i=1, \ldots, n
$$

Where $c$ is the joint centre in the global coordinate system, $\tilde{c}$ is the joint centre in local coordinate system at each time frame $i, \boldsymbol{R}_{i}$ is the rotation matrix that maps the global coordinate system to the local coordinate systems, and $t_{i}$ is the translation vector from the global origin to the origin of the local coordinate systems. Figure 3.4 illustrates all of these terms.

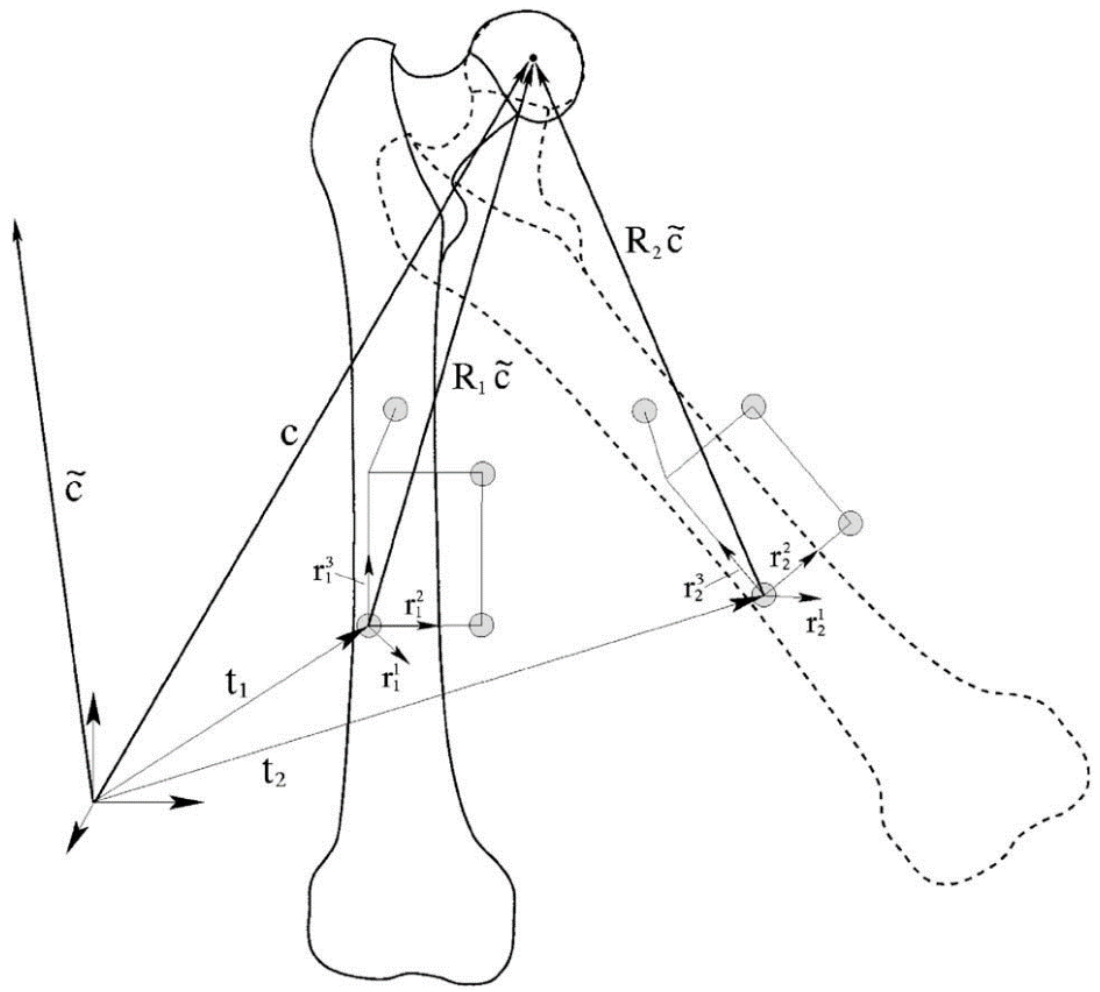

Figure 3.4 - Transformation of the Joint Centre between the Global and Local Coordinate Systems for the Femoral Segment [23] 
In order to calculate $c$ and $\tilde{c}$, the centre transformation technique attempts to minimize the residual of Equation (8), which leads to the objective function given by Equation (9).

$$
f_{C T T}(c, \tilde{c})=\sum_{i=1}^{n}\left\|\boldsymbol{R}_{i} \tilde{c}+t_{i}-c\right\|^{2}
$$

In order to minimize Equation (9), we can write it as a linear least square problem, which is expressed in Equation (10).

$$
\left(\begin{array}{cc}
R_{1} & -I \\
\vdots & \vdots \\
R_{n} & -I
\end{array}\right)\left(\begin{array}{l}
\tilde{c} \\
c
\end{array}\right)=-\left(\begin{array}{c}
t_{1} \\
\vdots \\
t_{n}
\end{array}\right)
$$

Where $I$ is a three dimensional identity matrix.

\section{- Symmetrical CoR Estimation}

The Symmetrical CoR estimation is the two sided technique introduced by Ehrig et al. [23]. The SCoRE technique considers the femur and pelvis in the CoR estimation. Local coordinate systems are defined for both segments and two centres are assumed for the segments, one for each of them. The position of the CoR is considered constant relative to both segments. To estimate the joint centre, the SCoRE technique minimizes the residual of the centres in the global coordinate system which is equivalent to the objective function given by Equation (11).

$$
f_{\text {SCORE }}\left(c_{1}, c_{2}\right)=\sum_{i=1}^{n}\left\|R_{i} c_{1}+t_{i}-\left(S_{i} c_{2}+d_{i}\right)\right\|^{2}
$$

Where $c_{1}, c_{2}$ are the joint centres of the pelvic and femoral segments in the local coordinate systems, $R_{i}, S_{i}$ are rotation matrices and $t_{i}, d_{i}$ are translation vectors to transform the local coordinate systems of the pelvis and femur to an appropriate global system. Equation (11) can be written as a linear least square problem that is given by Equation (12). 


$$
\left(\begin{array}{cc}
R_{1} & -S_{1} \\
\vdots & \vdots \\
R_{n} & -S_{n}
\end{array}\right)\left(\begin{array}{l}
c_{1} \\
c_{2}
\end{array}\right)=\left(\begin{array}{c}
d_{1}-t_{1} \\
\vdots \\
d_{n}-t_{n}
\end{array}\right)
$$

A variety of studies have compared sphere fitting methods and coordinate transformation techniques. Since the studies were performed under different conditions (different trial movements, human based studies, simulation based studies, etc.), their results didn't lead to clear conclusions about their methods. In general, coordinate transformation techniques have been reported to be more accurate and much less sensitive to noise compared to sphere fitting techniques. Ehrig et al. reported that the SCoRE algorithm is the most accurate transformation technique [7, 23].

\subsubsection{Summary}

Different studies aimed at comparing the accuracy of the methods to determine the HJC location. Leardini et al. compared the accuracy of sphere fitting methods with the predictive methods on eleven able bodied male subjects during several trials of hip rotation [6]. The sphere fitting methods were significantly better than the predictive methods. The error of sphere fitting methods was reported to be $13 \mathrm{~mm}$ however, the range was 25-30 mm for predictive methods. Hicks and Richards compared the accuracy of functional methods and predictive methods in determination of the HJC with the hip joint centre location from an ultrasound image on nine subjects [71]. The error associated with functional methods $(1.34 \mathrm{~cm})$ was significantly smaller than predictive methods $(2.16 \mathrm{~cm})$. According to the studies comparing the method to estimate HJC, some conclusions can be drawn:

- Image-based techniques suffer from an inherent error in determination of HJC location since they consider the femoral head as a sphere which is not a perfect sphere.

- Image-based techniques can be considered as a standard method for comparison with other techniques; but they are not commonly used to determine HJC location because MRI-based techniques are expensive and the other modalities in this category are invasive because of radiation exposure. 
- The accuracy of predictive methods depends on the identification of the anatomical landmarks.

- Several studies concluded that the performance of functional methods is better than predictive methods.

- Functional methods assume that the distance between the markers attached to the femur and the centre of rotation is invariant during the movement and their accuracy depends on marker's locations, ranges of motion, and types of movement.

- The main limitation of functional methods is soft tissue artefact due to skin deformation and muscle contraction.

\subsection{Soft Tissue Artefact}

Calculation of the hip joint functional centre based on relative motion of femur and pelvis is a key component of biomechanical motion analysis of human joint. According to the previous chapter, one of the non-invasive and most common used techniques for recording hip ranges of motion is optical motion capture systems. Optical motion capture systems involve the recording of reflective markers attached to the skin surface and they don't have direct access to the internal bone. The main limitation of optical systems in functional HJC location determination is relative motion between the markers and the bone because all functional methods consider the distance between the markers and the centre of rotation invariant during all movements. These relative motions between the markers and the bone are due to the muscle activity, as shown in Figure 3.4. This movement is known as Soft Tissue Artefact (STA), which is the most critical source of error in the estimation of the HJC location [11, 72]. 


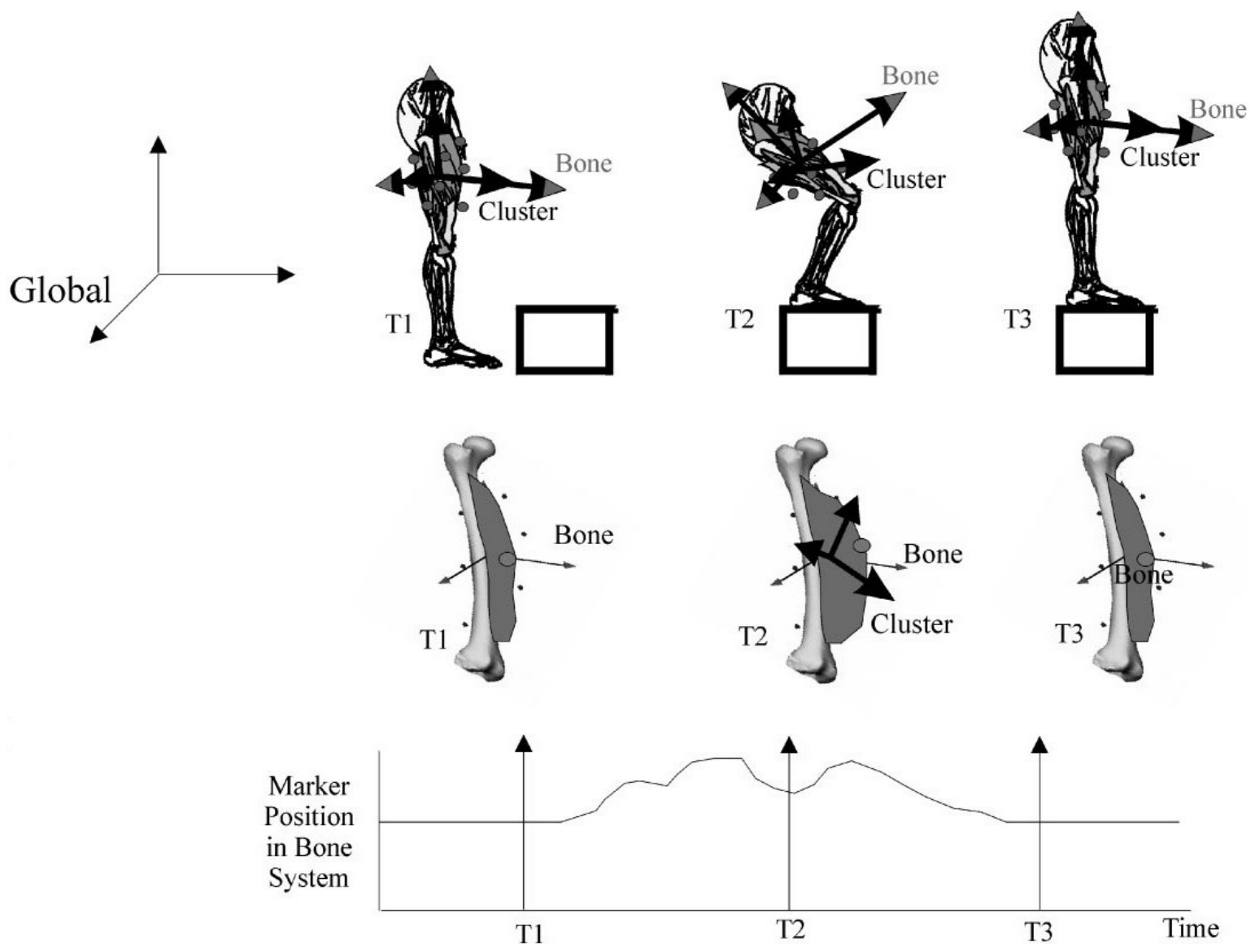

Figure 3.5 - The Marker Displacement in the Bone System during an Activity [16]

In order to have an accurate $\mathrm{HJC}$ and consequently accurate human motion analysis, the relative motion between the markers and the skeleton should be reduced. To reduce STA effects, it is crucial to understand STA patterns and magnitudes. Techniques to assess STA and minimize it in human motion analysis will be covered later in this chapter.

\subsection{Soft Tissue Artefact Assessment}

Several studies have described patterns and magnitudes of STA by different techniques. These studies can be categorized, by the techniques used, into five categories: intracortical pins, external fixators, percutaneous trackers, radiographic examinations and magnetic resonance imaging. In the following subsections, a summary description of the technique is provided. 


\subsubsection{Intra-cortical Pins}

Intra-cortical pins have been used in several studies to quantify STA magnitude. In 1991, a study based on intra-cortical pins was performed by Lafortune and Lake [73]. They used X-ray video fluoroscopy and intra-cortical pins to quantify STA during two experiments: three cycles of flexion/extension of the knee and heel strike during running. The results of the knee activity showed that a marker attached to the proximal tibia had a $21 \mathrm{~mm}$ distal and $23 \mathrm{~mm}$ posterior displacement that was linearly dependent on knee flexion angle. In the second experiment, $10 \mathrm{~mm}$ of relative movement was measured between a marker attached to a pin inserted into the tibia and another fixed to the skin of the lateral tibial condyle.

Karlsson and Lundberg performed a study on knee joint rotation of two subjects using intra-cortical pins and three reflective markers on an aluminum tripod [74]. The pins were implanted in the distal femur and proximal tibia and aluminum tripods were also attached to the distal thigh and the proximal shank. While the subjects were performing hip internal-external rotation with an extended knee, knee joint rotations were measured with bone pins and skin markers. The knee internal-external rotation range from bone pins was approximately $20^{\circ}$, however, the range was $50^{\circ}$ based on skin markers. Displacement of the thigh skin markers was bigger than the shank skin markers.

Reinschmidt et al. evaluated 3D rotations of the knee and ankle joints of five subjects during walking based on bone pins and skin markers [35]. Intra-cortical bone pins with a set of three reflective markers were inserted into the femur, tibia and calcaneus of the subjects. Six skin markers were attached to the thigh, six to the shank, and three to the foot. The results of three walking trials for each subject showed that the thigh markers mainly caused the errors in knee rotations and the foot markers caused the errors in ankle rotations. In another study, the same authors investigated the rotations of the knee joint of

three subjects during running activities using the same method as their previous study [38]. Errors in knee rotations based on shank markers were less than $5^{\circ}$, however errors based on thigh markers reached more than $10^{\circ}$. Also, the comparison of these two studies showed that the magnitude of skin movement errors were bigger during running activities than walking activities. 
Fuller et al. analyzed STA during several motor tasks using twenty skin markers attached to the thigh of one subject with bone pins inserted into the tibial tubercle and the greater trochanter [75]. Six markers were fixed to each bone pins. Skin markers displacements were task-dependent and reached $20 \mathrm{~mm}$.

\subsubsection{External Fixators}

External fixation devices can be used to quantify STA because they provide a set of axes associated with the underlying bone. Angeloni et al. analyzed STA using external fixators on the femur and tibia and video-fluoroscopic analysis during walking [76]. Several markers were placed on the skin surface and rigid plates were attached to the proximal half of the thigh and shank. Markers attached to the skin surface were on the greater trochanter, lateral epicondyle, head of the fibula, and lateral malleolus. The results showed that the STA associated with skin-mounted markers are larger than the ones on the rigid plates.

In a later paper by the same authors, markers displacements were computed by the same technique [39]. They used the fixator-markers to determine the coordinate system associated with rigid body and then compared the positions and orientations of the bone in this coordinate system with the coordinate systems defined from skin surface markers. Several motor tasks of the lower limb of five subjects were analyzed; these activities contain walking, cycling, flexion, extension, and rotation of the hip. STA associated with the markers on the anatomical landmarks depended on the joint angle and was independent of the type of activity. The drawbacks of external fixators are that they affect the normal movement of the skin and restrict normal joint motion.

\subsubsection{Percutaneous Trackers}

Percutaneous trackers are metal devices which are attached to bony segments of the body by several halo pins inserted into the opposite sides of the segments [40]. These devices have been used in some studies to quantify STA. Holden et al. measured the STA of three subjects by tracking the motion of the shank during walking [40]. They attached markers to the surface of the mid-shank and a percutaneous skeletal tracker to the distal tibia and fibula. Then, skin-based and skeletal-based anatomical frames (AF) were determined and 
the relative 3D difference between them was considered as the STA. It has been reported that maximum displacements of the skin-based AF were $10.5 \mathrm{~mm}$ in the longitudinal axis and $6 \mathrm{~mm}$ in the transverse plane.

Another study was performed by Manal et al. to obtain an optimal set of markers for motion tracking of the tibia on three subjects during walking [77]. This study provided information on STA and obtained similar results as the previous study. Subjects were asked to perform several walking trials while eleven different markers configurations were evaluated. Combining geometry, location (proximal/ distal) and attachment (underwrap/ overwrap) factors for the array made these different configurations. An optimal set of markers was observed for an underwrapped rigid shell, with four markers located distally.

\subsubsection{Radiographic Examinations}

Several studies have assessed STA using techniques based on radiographic examinations: Roentgen photogrammetry and fluoroscopy. 2D Rontgen photogrammetry was first used by Maslen and Ackland to quantify the relative skin movement of the foot and ankle of ten subjects during standing [78]. In order to track the skin movement, small steel markers were attached to the lateral malleolus, medial malleolus, navicular tuberosity, base of the fifth metatarsal, and sustentaculum tali. The mean displacement between skin markers and the underlying bones were 2.7-14.9 $\mathrm{mm}$.

In 1996, standard fluoroscopy was first used by Sati et al. to measurement the relative movement between skin and underlying bone at knee of three male subjects [79]. The medial and lateral parts of the subject's distal thigh were equipped with small metallic markers to track movement. Fluoroscopic images were collected while the subjects were flexing their knee approximately $65^{\circ}$. The magnitude of the movement was bigger for markers in locations closer to the joint line. Lateral and medial marker movement varied from 2.5 to $16.8 \mathrm{~mm}$ and 2.1 to $17.1 \mathrm{~mm}$, respectively.

Skin motion artefacts at the knee were assessed by using a biplanar radiographic system on two subjects during single leg forward hopping [80]. During knee surgery, three 1.6 mm diameter tantalum beads were implanted in the subject's tibia and femur. Two skin 
markers were attached to the medial and lateral epicondyles to measure the skin motion. The STA range was $5 \mathrm{~mm}$ to $31 \mathrm{~mm}$.

Another study assessed STA at the knee for two people who were getting total knee replacements [81]. This assessment used a combination of 3D fluoroscopy and stereophotogrammetry during stair climbing, step up/down, sit-to-stand/stand-to-sit, and extension against gravity. The subject's thigh and shank were equipped by nineteen and ten reflective markers respectively, which were distributed uniformly. Soft tissue artefacts were quantified by comparing the results of tracking the motion of theses skin markers by optoelectronic stereophotogrammetry with the 3D kinematics of the tibia and femur from the tracking the relevant prosthesis components by the fluoroscopy. The results revealed that the STA was task dependent, with the greatest impact on abduction/adduction and internal-external rotation angles. Also, the STA associated with the thigh, $31 \mathrm{~mm}$, was larger than the shank, $21 \mathrm{~mm}$.

\subsubsection{Magnetic Resonance Imaging}

Recently, magnetic resonance imaging (MRI) has been used in biomechanical analyses. Magnetic resonance imaging is another technique to quantify STA. Yahia-Cherif et al. used MRI to measure markers displacements and determine the best skin markers configuration for using in hip joint kinematics studies that use optical motion capture systems [13]. Nine reflective markers injected with a contrast agent, were attached at specific anatomical locations to the thigh skin of two subjects. Motion of the bone and markers were tracked in dynamic MRI while the subjects performed hip internal rotation, external rotation, flexion, extension, abduction, and adduction. Markers displacements were obtained by analyzing the markers trajectories versus bone trajectory in the MRI images. According to the displacements of all markers during the movements, the best markers configuration can be selected to have minimum STA during hip joint movement. The procedure for selection of the optimized markers configuration is explained in the soft tissue artefacts minimization section. In another paper, Yahia-Cherif et al. statistically quantified thigh STA as a function of hip anatomical angles on ten subjects [82]. It was shown that the markers displacements in the Z-direction were strongly 
correlated with hip internal-external rotation; and the markers displacements in $\mathrm{Y}$ direction were correlated with the hip flexion and extension.

Another study was performed by Sangueux et al. to quantify the 3D relative movement between skin markers and underlying bone in knee kinematics, based on MRI technique [12]. MRI measurements were acquired from right knee of eleven subjects during knee extension while the subjects laid in a supine position on the table in a MRI tunnel. Reflective markers injected with a contrast agent were placed on the subjects' femur and tibia and a foot derive device was used to have a reproducible range of motion of the knee was attached to the subjects' ankles. The results showed that the femoral marker displacements increased with the flexion angle and ranged from 3-22 mm. However, markers displacements of the tibia are almost independent of the angle in range of 1-9 $\mathrm{mm}$. These markers movements relative to the underlying bones affected the analysis of the knee kinematics.

\subsubsection{Dynamic Ultrasound Capture}

A method has been recently developed by Upadhyaya et al. [22] that combines optical motion capture and ultrasound imaging into a single experiment (and is essentially noninvasive); the stated advantage of this method is that it can capture the depth, due to STA, during dynamic motion. The drawback of the experimental setup is that it requires the

probe to be held in place (and perpendicular) by the participant while attempting to assure that the probe does not influence the skin/muscle deformation; this is extremely difficult and the experimental results show large margins for error. This method has the potential to produce clear information on the STA during motion, but it would at least require the use of a lightweight probe attached to the skin and this technology is currently not available.

\subsubsection{Summary}

In the previous subsections, a summary of studies on STA quantification for thigh and shank was provided. Techniques based on intra-cortical pins, external fixators, and percutaneous trackers can represent relatively accurate measurements of bone motion; but the use of these techniques is limited since the procedures are invasive. The error in 
techniques based on intra-cortical was caused be movement of the pins in the bones. The error in techniques based on external fixators and percutaneous trackers occurs due to movement restriction of the bone and joint which is caused by using these devices. The main drawbacks of techniques based on radiographic examinations are these methods are invasive by cause of radiation exposure, the 3D measurements of the STA are estimated from two planes which provide $2 \mathrm{D}$ information, and these techniques require extensive processing of image data [12]. Other non-invasive methods that have been developed are not sufficiently reliable.

The overall conclusions from the all studies on STA assessment are: the STA pattern is subject and task dependent, the STA at the thigh is larger than any other segments of the lower extremities, and this artefact is more considerable close to the joints [11].

\subsection{Soft Tissue Artefact Minimization}

According to the previous section, the relative movement between the skin markers and the underlying bone is significant. STA has a great impact on human motion analysis, particularly hip joint kinematics as STA associated with the thigh is larger than any other lower extremities. Therefore, it is necessary to minimize its effects. Several methods have been proposed to reduce the STA effects: solidification model, multiple anatomical landmark calibration, pliant surface modeling, dynamic calibration, point cluster technique, global optimization, and techniques based on MRI. In the following subsections, a brief description of each approach is provided.

\subsubsection{Solidification Model}

Cheze et al. developed a two-step solidification procedure to decrease the displacement of the markers attached to the skin [14]. Since three non-collinear markers are sufficient to determine a segment's position and orientation, the first step of this procedure is seeking for three markers which present the least perturbed triangle during the entire movement among the markers located on the segment. Once the best three markers have been indicated, the solid triangle shape which fits this time varying triangle appropriately is defined from the measured triangles. This procedure is performed through iterative and 
elimination procedures. During these procedures, the mean values for each of the three vertex angles are calculated and the largest one corresponded to the most deformed frame is found and eliminated. The purpose of this process is elimination of extremely deformed frames and is repeated until $75 \%$ of the frames are retained. The second step is fitting the best solid triangle to each measured triangle at each point in the motion using the standard singular value decomposition (SVD) algorithm. The authors validated their method in knee kinematics using the trajectories of markers attached rigidly to the thigh and shank based on experimental data from swing phase of gait. In order to obtain perturbed trajectories of the markers, a continuous noise model representing typical skin movement errors with the form $A \sin (\omega t+\varphi)$ was applied to the markers trajectories; where $A$ is the amplitude of the noise, $\omega$ the frequency, $t$ the simulated time, and $\varphi$ the phase angle. The solidification model doesn't reduce kinematic errors better than leastsquares method as it can only identify erroneous frames.

\subsubsection{Multiple Anatomical Landmark Calibration}

In human motion studies, one of the fundamental steps is calibrating ALs to find out various aspects of marker configuration and skeletal segments. Multiple anatomical landmark calibration considers STA as a parameter related to movement of ALs relative to a technical reference frame defined by markers attached to the skin. Cappozzo et al. presented a method to reduce the errors in estimation of ALs in technical frames [58]. This method is known as Calibrated Anatomical System Technique (CAST) and used as a standard anatomical calibration technique. The method consists of a static calibration of ALs in a technical reference frame defining by skin markers. To this aim, several markers are placed on the segment skin, except the ALs locations to define the technical reference frame. Then, the local position of each AL is determined in the technical reference frame by using a pointer on which a minimum of two markers have been mounted in known positions. Then, at least one frame is recorded and the procedure is repeated for each AL. Using obvious geometric calculations, the locations of ALs are determined and thus the anatomical frames can be determined. This method improves the locations of ALs which leads to an improvement in estimation of position and orientation of the bones during a motor task. 
In another study, Cappello et al. improved the previous method [83]. They proposed a double calibration of the ALs at the two extremes of the expected range of motion a specific motor task. In their method, movements of skin are considered linear relative to the underlying bone. The AL locations between two motions are computed by linear interpolation in time. This method was validated on a subject wearing a femoral external fixator during a cycling exercise. The results showed that the reconstruction error for an $\mathrm{AL}$ (greater trochanter) was decreased from $15 \mathrm{~mm}$ to less than $10 \mathrm{~mm}$. The errors in femoral motion, femur orientation and position, were reduced from 5 and $7 \mathrm{~mm}$ to less than 4 and $4.5 \mathrm{~mm}$, respectively. Although multiple anatomical calibration techniques are able to reduce errors in pose estimation, they are subject specific which makes them inappropriate for daily clinical uses.

\subsubsection{Pliant Surface Modeling}

In traditional movement analysis, the human body is considered as a rigid segment with 6 DoF (3 rotations and 3 translations), however pliant surface modeling (PSM) is a nonrigid model that considers 12 DOF per body segment, 6 additional DoF ( 3 scales and 3 shears) compared to traditional rigid body modeling (RBM) [84]. Pliant surface modeling quantifies displacements of the skin fixed markers due to skin stretching, muscle activities, and internal phenomena while it is quantifying rigid rotations and translations of the segment. The authors compared the performance of PSM technique with traditional RBM techniques. Experimental data of three able-bodied subjects were collected. Two pins were inserted into the femur (greater trochanter) and tibia (gerdy's tubercle) of each subject without having passed through thick soft tissues. In order to obtain real bone movements, a circular cluster of markers was placed on the top of each pin and on the shank and thigh surfaces. Kinematic data were collected while subjects walked on a treadmill at three different speeds. The results revealed that the pliant surface model provided more accurate estimation of femur and tibia positions. The error associated with PSM in this estimation is $3.8 \mathrm{~mm}$ compared to $4.8 \mathrm{~mm}$ with RBM. The improvement in segment orientation was not significant and was less than $0.5^{\circ}$. The drawback of the PSM procedure is that only affine deformations are measured. 


\subsubsection{Dynamic Anatomical Landmark Calibration}

Dynamic anatomical landmark calibration has been proposed by Lucchetti et al. to determine anatomical landmark positions in an ad hoc movement [85]. Dynamic anatomical landmark calibration assesses the relative movement of ALs in a technical reference frame to measure STA errors of skin markers. The method is a combination of experimental and analytical procedures to estimate knee STA of three subjects. One of the subjects was wearing a prosthetic knee that was used for validation of the method. Four markers were fixed on the pelvis by a rigid plate; five and four markers were attached to the thigh and shank, respectively. The procedure of ALs calibration is similar to Cappozzo et al. [58]. Cappozzo et al. calibrated ALs by a pointer at static posture in a motor task however Lucchetti et al. measured AL positions in an ad hoc movement. Motor tasks recorded in the method are upright posture, right hip flexion/extension followed by abduction/adduction with hyperextended knee, right lower limb forward swing with hyperextended knee, and level walking along a straight line. Technical frames were estimated to calculate AL positions and anatomical frames. Then, they derived a discrete function to model the relation between the displacement of ALs and joint angles in order to correct $\mathrm{AL}$ positions relative to technical reference frame during a motor task. This discrete function was used to reduce STA effects during walking. Errors in estimation of translations and orientations of the knee joint were reduced from $14 \mathrm{~mm}$ to $4 \mathrm{~mm}$ and from $6^{\circ}$ to $3^{\circ}$, respectively. The use of method in clinical applications is limited as it is time consuming due to additional acquisition and elaboration of movements.

\subsubsection{Point Cluster Technique}

Andriacchi et al. proposed a point cluster technique to minimize STA effects, which was based on the idea that eigenvalues of the inertia tensor of rigid body segments are time invariant [86]. A cluster of large number of markers was uniformly attached to the body segment under analysis. At each time frame, $t$, an arbitrary mass $m(t)_{i}$ was assigned to each marker $i$ and the centre of mass and inertia tensor were calculated. Then the 
eigenvalues and eigenvectors of the inertia tensor were computed. The mass of each marker was adjusted at each step to minimize the changes in eigenvalues.

If the location of the marker $i$ in the global coordinates system is considered as $\mathbf{G}(t)_{i}$, the centre of mass, $\mathbf{C}(t)$, and the inertia tensor are given by Equations (13) and (14), respectively.

$$
\begin{gathered}
\mathbf{G}(t)_{i}=\left[\begin{array}{l}
g(t)_{i, x} \\
g(t)_{i, y} \\
g(t)_{i, z}
\end{array}\right], \quad \mathbf{C}(t)=\frac{\sum_{i=1}^{n} \mathbf{G}(t)_{i} m(t)_{i}}{\sum_{i=1}^{n} m(t)_{i}}, \quad i=1, \ldots, n \quad(13) \\
\mathbf{I}(t)= \\
{\left[\begin{array}{ccc}
\sum_{i=1}^{n}\left[\left(p_{i, y}\right)^{2}+\left(p_{i, z}\right)^{2}\right] m(t)_{i} & \sum_{i=1}^{n} p_{i, x} p_{i, y}\left[-m(t)_{i}\right] & \sum_{i=1}^{n} p_{i, x} p_{i, z}\left[-m(t)_{i}\right] \\
\sum_{i=1}^{n} p_{i, x} p_{i, y}\left[-m(t)_{i}\right] & \sum_{i=1}^{n}\left[\left(p_{i, z}\right)^{2}+\left(p_{i, x}\right)^{2}\right] m(t)_{i} & \sum_{i=1}^{n} p_{i, y} p_{i, z}\left[-m(t)_{i}\right] \\
\sum_{i=1}^{n} p_{i, x} p_{i, z}\left[-m(t)_{i}\right] & \sum_{i=1}^{n} p_{i, y} p_{i, z}\left[-m(t)_{i}\right] & \sum_{i=1}^{n}\left[\left(p_{i, x}\right)^{2}+\left(p_{i, y}\right)^{2}\right] m(t)_{i}
\end{array}\right]} \\
\mathbf{P}(t)_{i}=\mathbf{G}(t)_{i}-\mathbf{C}(t), \quad i=1, \ldots, n
\end{gathered}
$$

The authors evaluated their methods in a simulation model and applied systematic and random errors to the markers. Results showed that the error due to non-rigid body movement could be significantly reduced. Also, they evaluated their method by applying it to ten normal subjects to obtain knee rotation and translation during walking. They compared the results with the results of another study using intra-cortical pins inserted into the femur and tibia of the subjects [36]. This comparison indicated that the point cluster technique performed well. The main limitation of point cluster technique is using a large number of markers, which leads to track and label the markers difficultly.

\subsubsection{Global Optimization}

All the previous mentioned techniques suffer from an inaccuracy due to treating each body segment separately without imposition of joint constraints contrary to the fact that STA can induce non-physiological joint translation and dislocation [11]. In order to 
overcome this limitation, $\mathrm{Lu}$ and $\mathrm{O}^{\prime}$ Connor proposed an optimization method which determined and optimized joint centre locations and segment orientations of multilink musculoskeletal models iteratively and globally while imposing joint constraints [18]. This global optimization is based on the minimization of the sum of squared distances between measured and model-determined marker positions which are weighted by weighting matrices throughout all the body segments. Weighting matrices reflect average degree of STA. Different weighting schemes are assigned to each segment but all markers of a segment are given equal weightings.

The method was evaluated with twenty computer-simulated gait trials considered as twenty different subjects with different patents of skin movements. An artificial noise was introduced to markers coordinates to have perturbed marker trajectories as in Cheze et al. [14]. The authors evaluated the performance of the method in terms of joint angles and joint centre locations. Skin movement artefacts in determination of joint rotations were largely reduced particularly in axial rotation and abduction/ adduction. Moreover, the dislocation of the joints was eliminated. The method wasn't assessed and validated in human studies; it was evaluated only on computer-simulated data. The drawback of the global optimization technique is that it simplifies joints structures, the assumption of balland-socket joints, which are not subject-specific and cannot be applied to people with hip joint disorders [18, 19].

\subsubsection{Magnetic Resonance Imaging}

Yahia-Cherif et al. presented a technique consisting of MRI to capture the anatomy and the trajectories of the bone in order to reduce STA effects in hip joint kinematics studies which use optical motion capture systems [82, 13]. Their method consists of using dynamic MRI to assess the displacements of the markers and select the best markers configuration in order to develop a correction algorithm to minimize the STA effects. To these aims, the authors used nine reflective markers injected with a contrast agent attached to the thigh skin of subjects at specific anatomical locations. Then, the motion of the bone and the markers were tracked in dynamic MRI while the subjects performed hip internal rotation, external rotation, flexion, extension, abduction, and adduction. The displacement of the markers was obtained by analyzing the marker trajectories versus 
bone trajectory in the images. After calculating markers trajectories, for each marker $m_{i}$, there is an error $r_{i}$ corresponding to the sum of the displacement of the marker from frame to frame. The sum of all markers frame-to-frame displacements is calculated. As three markers are sufficient for HJC determination, the algorithm searches three markers among all markers with specific criteria to obtain the optimized configuration. In order to choose the best three markers, first, all triplets of markers which are non-collinear are chosen. Then, the best triplet of markers is the one which the distance between its markers is the most one and the displacements of its markers are the less ones. For markers $m_{i}, m_{j}, m_{k}$ and their corresponding errors $r_{i}, r_{j}, r_{k}$, the total distance of three markers and the total error can be defined as $d_{i j k}=d\left(m_{i}, m_{j}\right)+d\left(m_{j}, m_{k}\right)+$ $d\left(m_{k}, m_{i}\right)$ and $r_{i j k}=r_{i}+r_{j}+r_{k}$, respectively. Therefore, the optimized configuration is determined by minimizing the $r_{i j k} / d_{i j k}$ through an exhaustive search. Although the authors didn't validate their method, the optimized configuration can be used in hip joint kinematics studies which use optical motion capture systems in order to reduce the STA errors.

\subsubsection{Summary}

In the previous subsections, we presented the existing methods to minimize and compensate the STA effects in motion analysis. The solidification model does not compensate the STA effects well as it can only identify erroneous frames [11, 14]. Dynamic calibration and multiple anatomical landmark calibration are based on invalid assumptions (linearity assumptions) and they are time consuming because they require additional data acquisitions [15]. The limitations of the point cluster technique are an overabundance of markers and instability [16, 17]. The drawback of the global optimization technique is that it simplifies joints structures that are not subject-specific which cannot be applied to people with hip joint disorders [18, 19]. MRI-based techniques require expensive medical imaging and they are not suitable for everyday clinical measurements and analyses. Most of the presented methods were validated for the knee joint only; therefore there is no strong evidence of their effectiveness in compensating the STA in the HJC estimation. 
Despite the numerous methods proposed, the objective of a reliable, non-invasive, and clinical estimation and correction of STA in human hip joint kinematics is still a topic of research and interest. In next chapter, we propose an improved method for assessing and eliminating the STA effects using optical motion capture analysis and ultrasound depth measurements. 


\section{Chapter 4: Developing a Method to Assess and Minimize Soft Tissue Artefact in Hip Joint Centre Estimation}

\subsection{Introduction}

We propose an improved method consisting of ultrasound measurements of tissue thickness and motion capture analysis to quantify and minimize STA non-invasively to determine the HJC using a functional method. Our solution is to first record each marker's position placed on the thigh and pelvis for a range of motions of the hip joint (standing, flexion, extension, and abduction). When the thigh moves, the muscles of the upper thigh area contract and relax which cause changes in the muscle thickness. These changes affect the positions of the markers attached to the skin relative to the underlying bone and introduce an STA error in the calculation of the HJC. We use ultrasound imaging to measure the changes in tissue thickness at the marker positions for the same standing and extended positions. The use of ultrasound imaging for the functional determination of the HJC was introduced by Sangeux et al. [20] and using it, in combination with motion capture as a validation tool, was introduced by Peters et al. [21]; however Upadhyaya et al. [22] first introduced the idea of combining both ultrasound and Motion Capture into a single analysis process, we have continued this trend for our method. This information of tissue thickness is used to select three markers 
having less change in their tissue thickness. These markers are considered as three key markers and will be further used in mathematical analysis on the data to assess and eliminate STA effects. Next step is fitting curves to the marker positions and applying UDM information in order to determine bone positions at the positions of three key markers. In fact, by determining these positions, we eliminate the error in markers positions caused by changes in tissue thickness. We use these positions on the bone to assess STA during several movements of the hip joint as the. Therefore, once the bone positions at three key markers of all motions of the hip joint (standing, flexion, extension, and abduction) have been determined, we attempt to find a rotation matrix and translation vector which transform the bone positions at three key markers of standing position to each of the other movement types as the bone is rigid body. By applying the matrix and the vector to the markers trajectories of standing position and comparing with the trajectories of markers of the other movement types, the STA can be quantified. The next step is the HJC calculation; we calculate the HJC using a coordinate transformation technique, SCoRE algorithm [23]. To have an accurate HJC location, we use the displacement of the markers from the previous step and recalculate the marker positions to eliminate STA effects used in the SCoRE algorithm. Our method is outlined in Figure 4.1 and each step is described in the following subsections. 


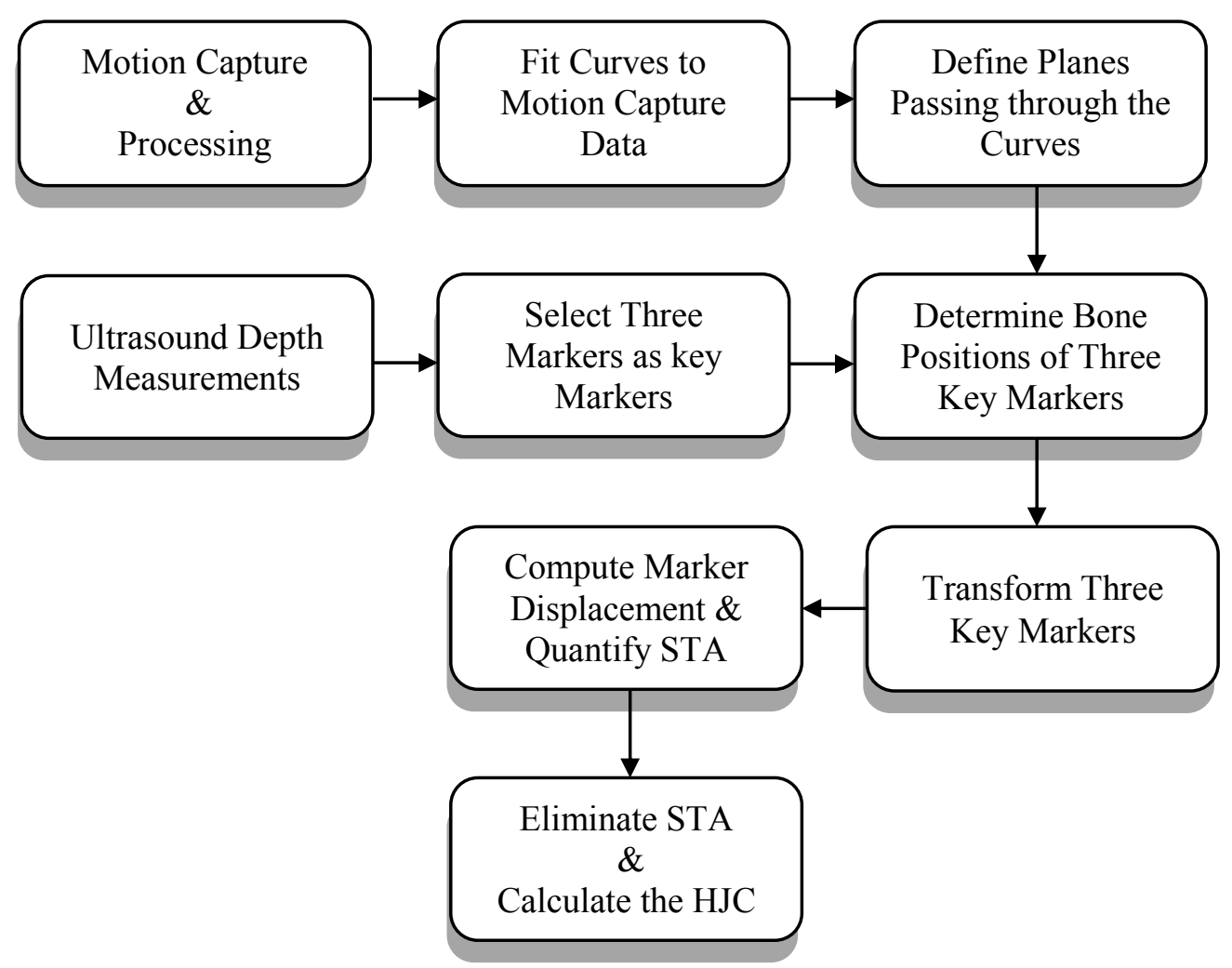

Figure 4.1 - Overall Process for STA Assessment and Compensation

\subsection{Optical Motion Capture}

Optical motion capture systems are the most used systems in human movement studies. These systems record realistic motions of human bodies in high detail, even for complex motions, while they are non-invasive and have no harm to human body. Optical motion capture systems track reflective markers attached to the skin by cameras. Our optical motion capture system is a Vicon MX system consisting of 10 wall-mounted nearinfrared cameras. The subject is surrounded by the cameras while small reflective markers are placed on the skin surface. The cameras transfer the 2D information of the markers trajectories to a computer. The information is obtained by emitting near-infrared lights from the cameras on the reflective markers and their reflection to the cameras. Figure 4.2 and Figure 4.3 illustrate our motion capture lab and one vicon wall-mounted near-infrared camera, respectively. 


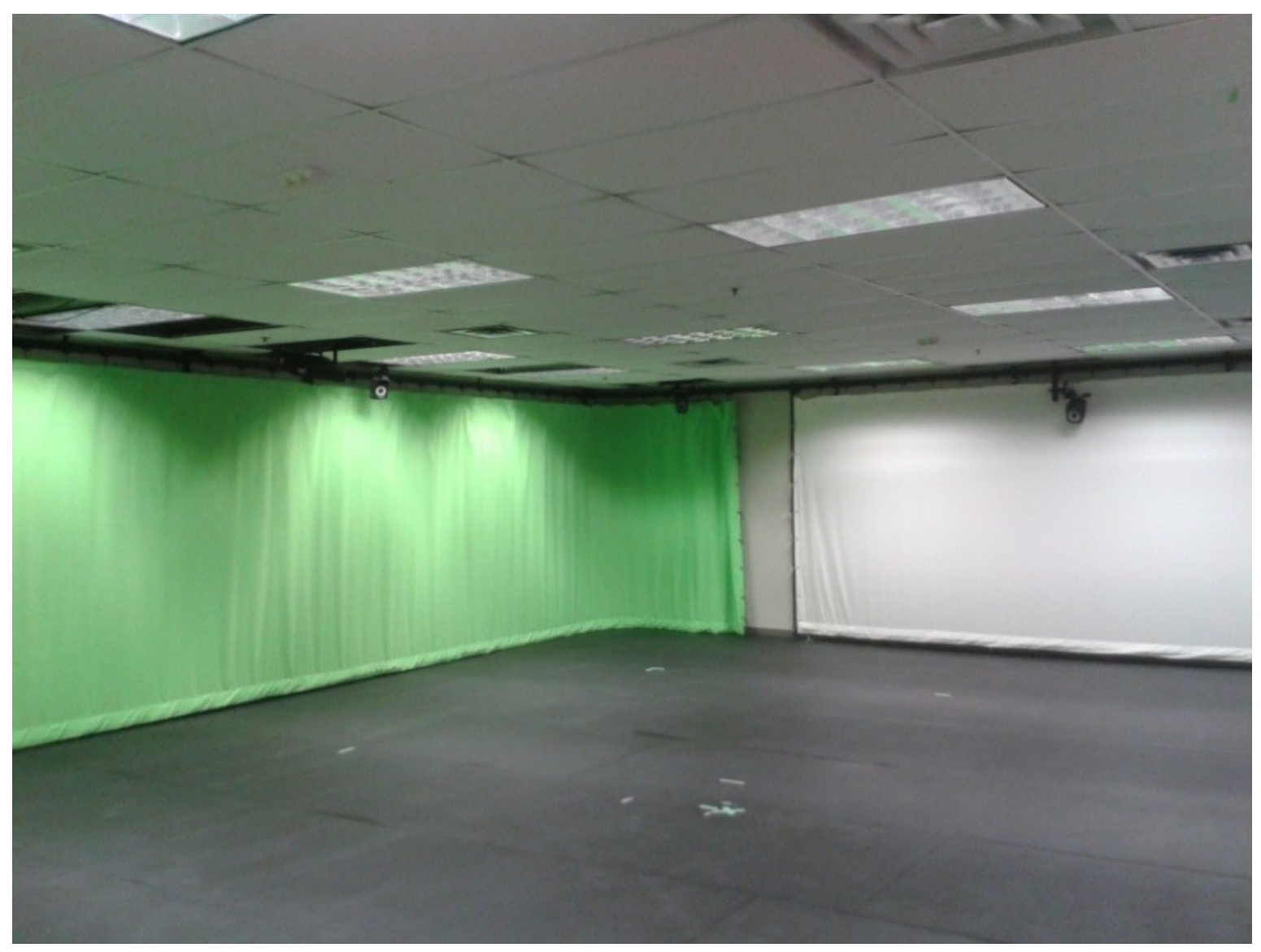

Figure 4.2 - Motion Capture Lab

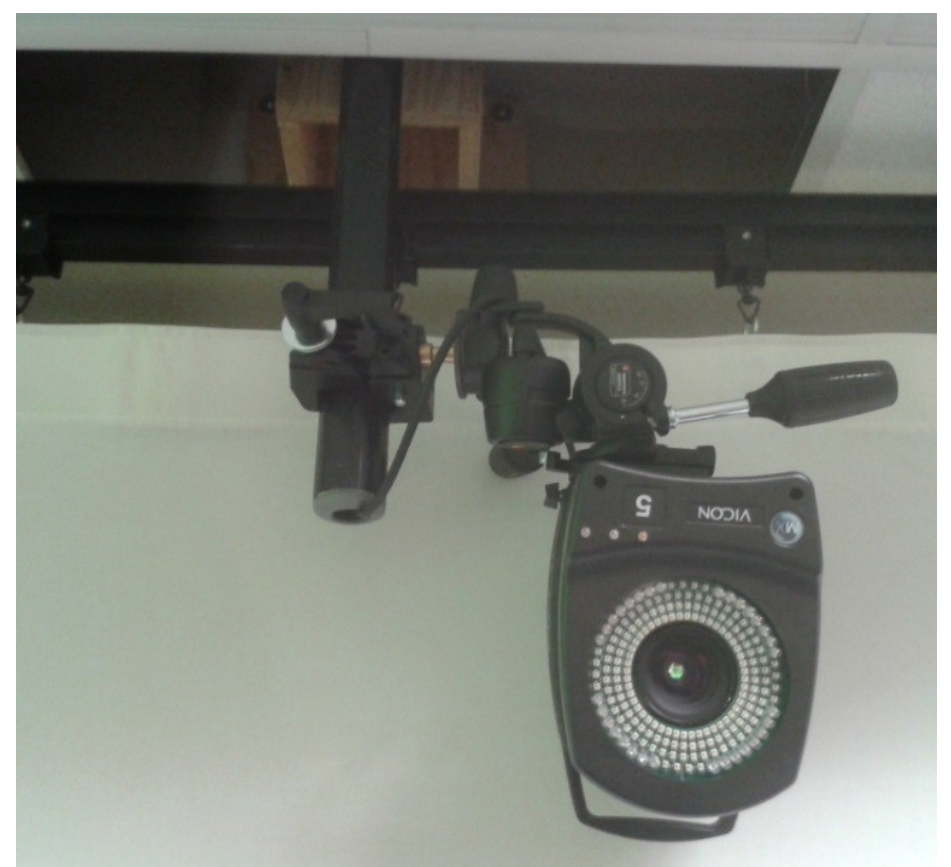

Figure 4.3 - Vicon Wall-mounted Near-infrared Camera 
The 2D information of marker trajectories from 10 cameras is combined to have their $3 \mathrm{D}$ information. Each session of our optical motion capture consists of four stages: calibration of the system, reflective markers placement, motion tracking and data postprocessing. These stages are explained in the following subsections. In order to provide the description of optical motion capture section, Carleton guide website to use Vicon optical motion capture system was used [87].

\subsubsection{Calibration of System}

An essential stage in optical motion capture is calibration of the system before starting recording any motions. The system needs to be calibrated in order to measure the positions and orientations of the cameras relative to each other and the lab's coordinate system. Calibration of the system involves two steps: static calibration and dynamic calibration. Static calibration sets the world origin and the global axes of the motion capture volume. This step is performed by using an L-Frame as shown in Figure 4.4a, the L-Frame consists of four reflective markers to set the origin and the axes. Dynamic calibration sets the locations and orientations of each camera according to the other cameras. Dynamic calibration is performed by a wand with three markers while the wand (as shown in Figure 4.4b) is "waved" around the area of capture like a painting process of that volume. 


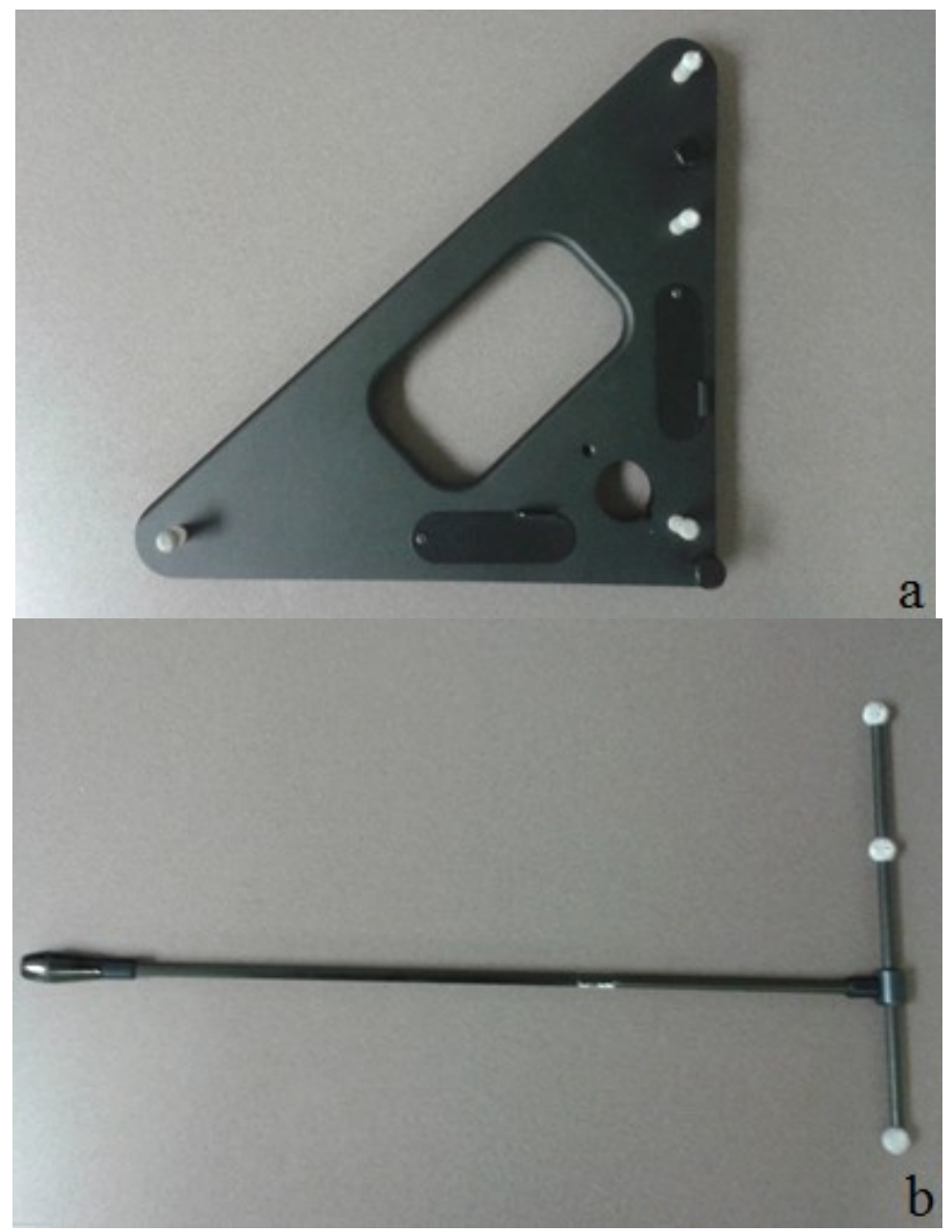

Figure 4.4 - Calibration of the Vicon system: a) L-Frame b) Wand

Vicon MX system uses system calibration information to create 3D trajectories of the markers from the 2D trajectories received from all 10 cameras.

\subsubsection{Markers Configuration}

To capture the movement of the hip joint, we use two groups of markers attached to the skin of the subjects. The first group of markers consists of 8 spherical reflective ones at palpable bony landmarks where the bone is very close to the skin surface and thus the soft tissue artefact is minimal. These locations include three on the hip area, left and right anterior superior iliac spine and the lower spine, two on either side of the knee, medial and lateral femoral epicondyles, and two on either side of the ankle, medial and lateral malleolus, and one on greater trochanter. As our goal is soft tissue artefact assessment in hip joint kinematics, the other group consists of the markers which are distributed over 
the skin of the thigh. These markers are affixed to the skin surface of the subjects in four ring formations. The rings are placed approximately $5 \mathrm{~cm}$ apart, with eight markers per ring. These positions are marked on the thigh and used for the ultrasound depth measurements in the second stage of our experiments in this thesis. The markers configuration of the thigh is illustrated in Figure 4.5.

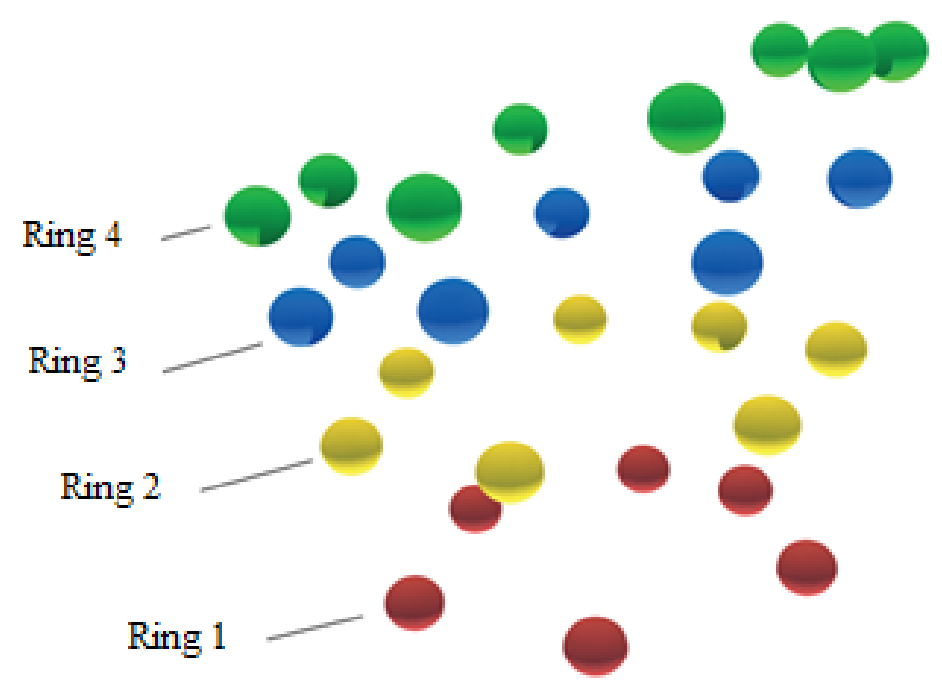

Figure 4.5 - Skin Markers Configuration

\subsubsection{Motion Tracking}

Once the markers have been attached to the subject's skin surface, we can capture and track the hip joint movements. The first step in our motion tracking is capturing the markers trajectories in standing position as a reference for subsequent processing. Participants are requested to move their leg that is equipped with the reflective markers in three key motions, flexion, extension, and abduction, starting from a standing position. Markers trajectories are captured for these positions as shown in Figure 4.6. To have the same range of motion of the hip joint for ultrasound depth measurements, the positions are determined using non-reflective blocks that are setup ahead of capture with a specific configured distance. 


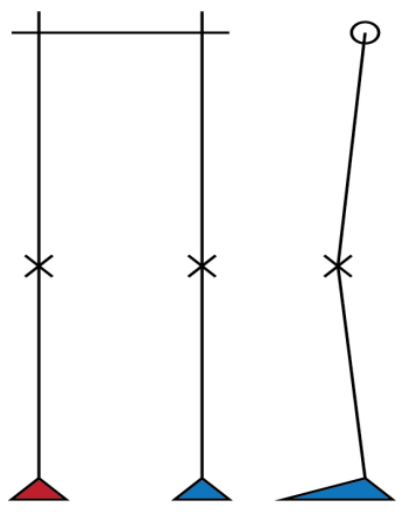

Standing Position

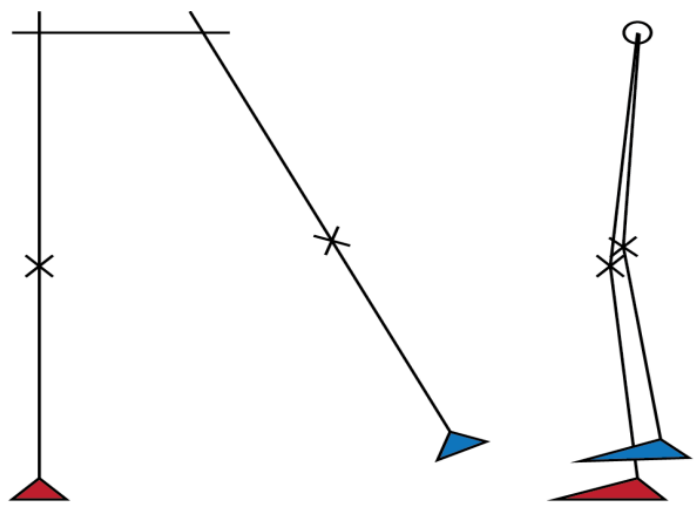

Abduction Position

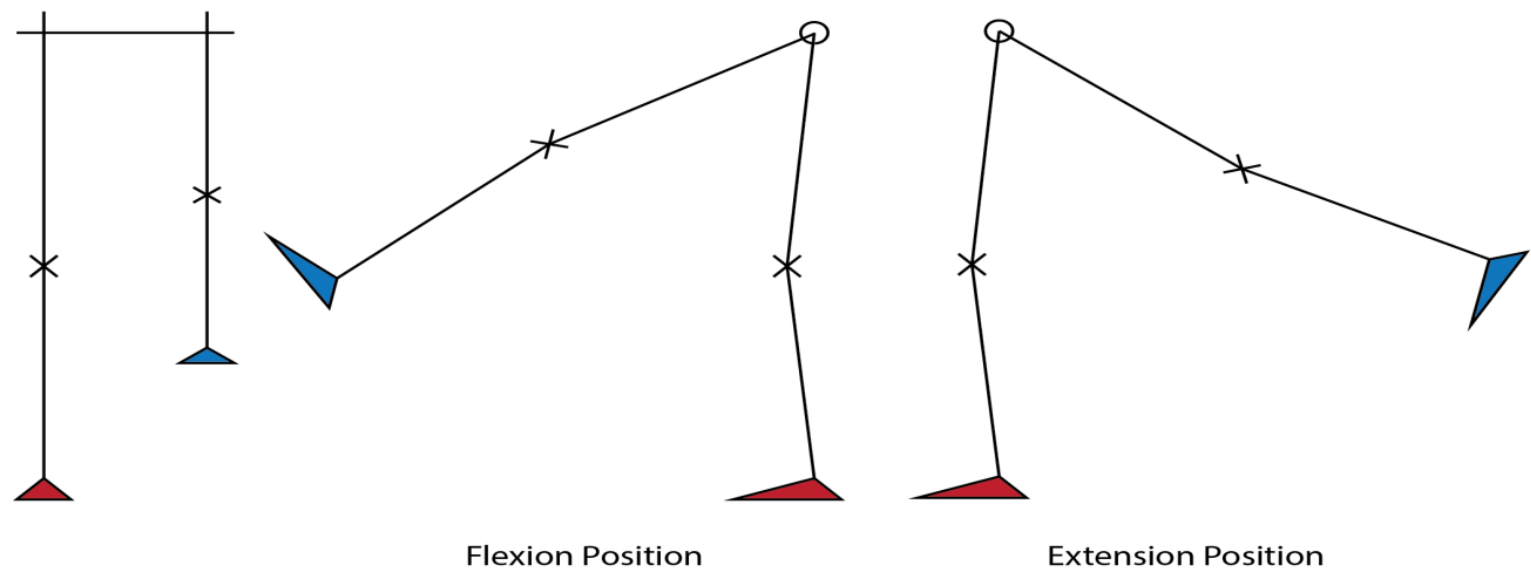

Figure 4.6 - Subject Positions during Trial, Abduction, Flexion, and Extension

\subsubsection{Data Post-processing}

The last stage in our optical motion capture session is data post-processing, after recording the motions. The first step in data post-processing phase is to reconstruct the 3D positions of all markers during the movements. For each type of movements, the 3D positions of the markers are represented in $N$ frames. In order to subsequent processing of the $3 \mathrm{D}$ positional information of the markers, we need to select proper frames of the movements and label the markers. Once the proper frames have been chosen and the markers have been labeled, the markers trajectories are exported to a C3D file format to be used in MATLab for next processing of motion capture data. The C3D file format is a common file format in movement analysis applications that stores 3D positional information of the markers. 
Next step in our study is ultrasound depth measurements to combine them with the information from motion capture to assess the STA which is explained in the next sections.

\subsection{Ultrasound Imaging}

Ultrasound is one of the preferred imaging modalities because this modality is noninvasive and poses no harm to human bodies and, in addition, it is a low cost and portable imaging modality. In our proposed method to improve the determination of the HJC location, ultrasound imaging is used to measure the tissue thickness.

Ultrasound refers to sound waves with high frequencies in the range between $20 \mathrm{KHz}$ and $1 \mathrm{GHz}$ which is above the human hearing range [88]. Clinical ultrasound imaging uses these high frequency acoustic signals, typically between 1 and $10 \mathrm{MHz}$, to produce images of internal structures of the body non-invasively. A main part of ultrasound imaging systems is a transducer probe which generates and transmits the ultrasound into the tissues of the body and received the reflecting echoes back [89]. As the transmitted echoes pass through the tissues, they hit boundaries between the tissues and lose some of their energy. Penetration depth of the transmitted signal through the body is dependent on its frequency. Some parts of transmitted echoes are returned back to the transducer probe after reflecting from different levels of tissues boundaries while the echoes pass through the body. The transducer probe converts the reflected acoustic signals to electrical signals and processes the echoes based on the distances of the tissue boundaries from the transducer probe using the speed of the transmitted echoes and the arrival time of the reflected echoes to the transducer probe. By processing the signals received at the transducer probe, ultrasound imaging systems can display a two dimensional grayscale image of the tissues. The image pixels represent organs locations which reflected the echoes and the brightness of the pixels is dependent the echoes strength [90,91].

Figure 4.7 and 4.8 show the ultrasound imaging machine and ultrasound transducer probe used in this study, respectively. 


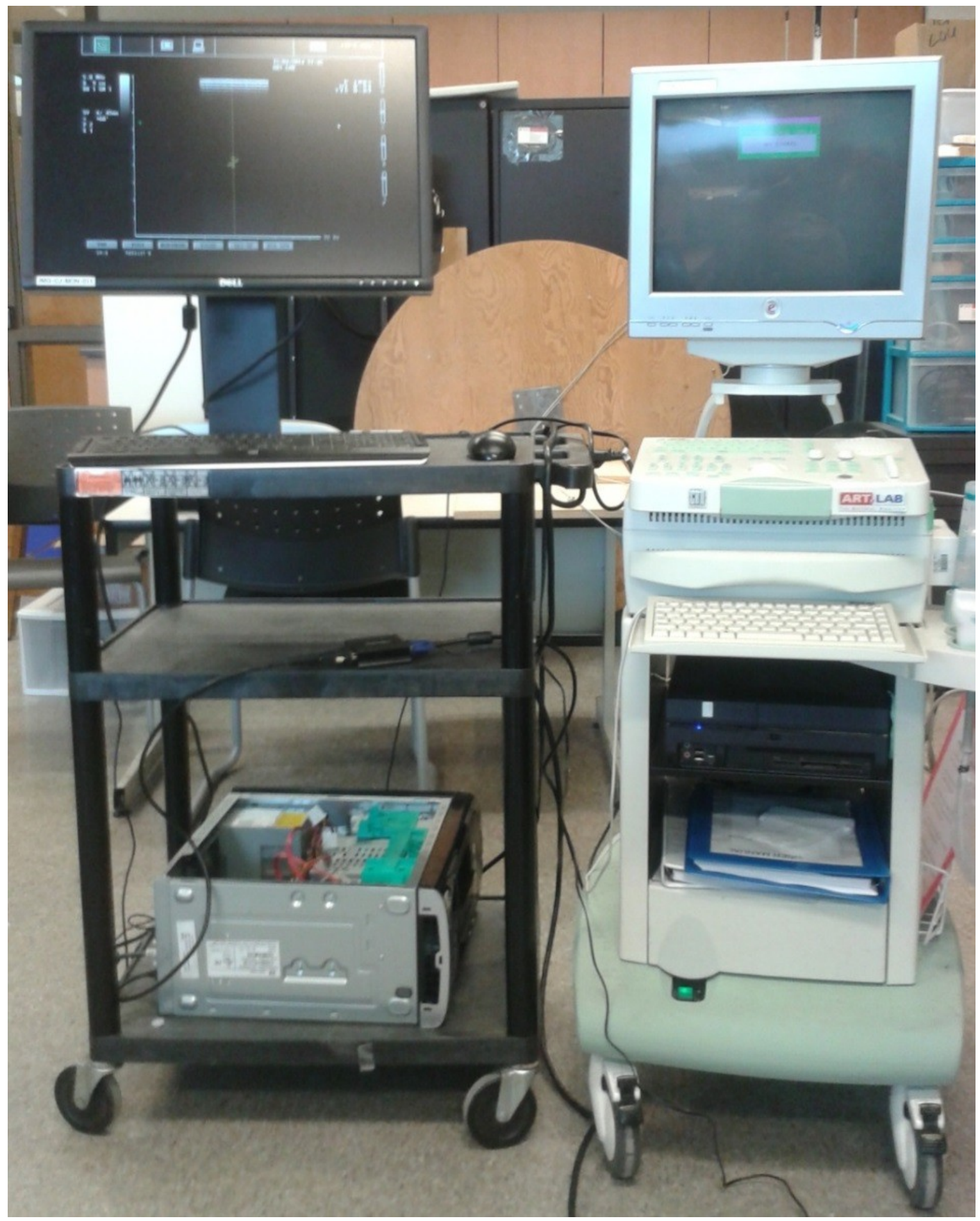

Figure 4.7 - Ultrasound Imaging Machine 


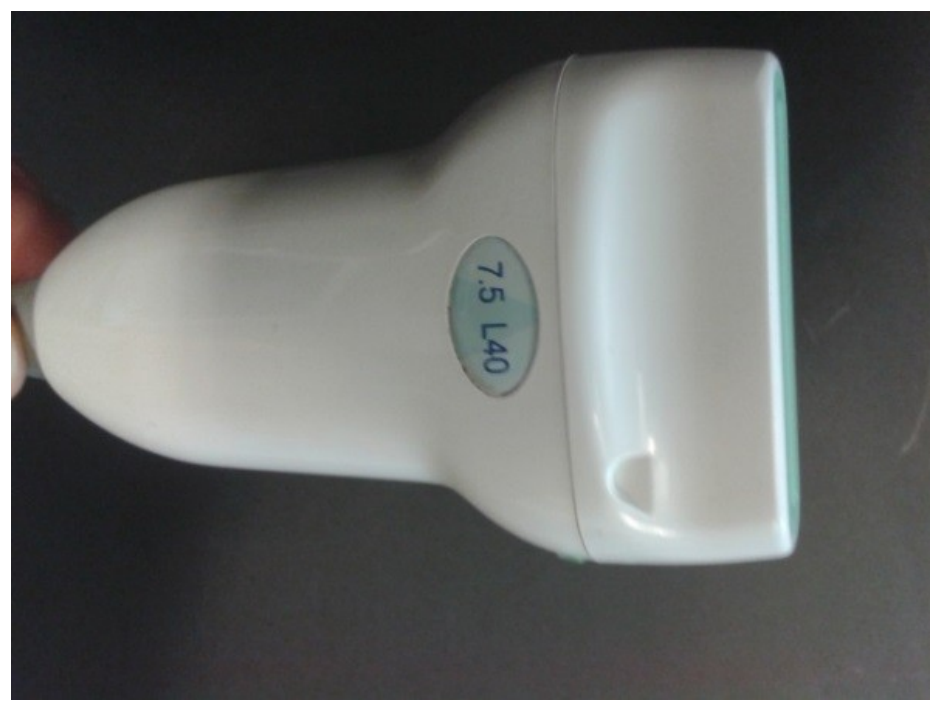

Figure 4.8 - Ultrasound Transducer Probe

The transducer probe shape (linear, convex, phased array and etc.) determines its field of view of the body. Our ultrasound probe is a linear type that keeps same field of view at different depths of the subject's body.

\subsubsection{Edge Detection in Ultrasound Images}

Using the ultrasound imaging to measure tissue thickness from the bone position, ultrasound echoes pass through tissues. Since soft tissues and the underlying bone having different acoustic impedances, their reflected echoes are different. In fact, ultrasound echoes reflected from the bone surface are very strong and cause high intensity pixels in the image representing the bone surface. Detecting the desired edges in ultrasound images is not easy since they are extremely noisy and consist of various artefacts and unrelated high contrast noise. In our application, the echoes reflected from the layered structures of different muscles cause relatively high intensity pixels and consequently error in detecting the desired bone surface. When we observe unrelated edges that make the bone surface detection difficult, we give a little push to the ultrasound probe to distinguish the unrelated edges. As mentioned, these unrelated edges are caused by the layered structures of different muscles; therefore pushing the ultrasound probe changes the thickness of the muscles and structures of corresponding high intensity pixels in the image however the pixels correspond to the bone surface are not changed. After detecting the desired edge, the probe is released to measure the real thickness of soft tissues. 


\subsubsection{Determine Three Key Markers}

Once the tissue thickness of the indicated markers on the thigh has been measured, we need to find three of the markers that have less depth changes during all positions (standing, flexion, extension, and abduction). To this aim, the coefficient of variation (CV) of each marker's depth measurements during all positions is obtained and three markers with less value of CV will be selected as three key markers for next steps of our method. Since CV measures relative variability and describes the variation relative to mean of a set of data, it is useful to compare data variation among two or more sets of data. The low value of the CV shows that the dispersion in the variable of a set of data is not great.

The coefficient of variation of each marker is calculated using the following equation.

$$
C V=\frac{S}{\mu}
$$

Where $S=\sum_{i=1}^{4}\left(D_{i}-\mu\right)^{2} / 3, \mu=\sum_{i=1}^{4} D_{i} / 4$ and $D_{i}$ is the depth measurements for all four positions.

\subsection{Data Analysis}

In this section, we explain our proposed approach consisting of four steps to analyze data for STA assessment. The first step is passing a curve through the ring formation of each of the key markers. The second step is defining planes passing through the curves from the previous step. Then we propose a mathematical method to determine the projection of the key markers on the underlying bone. These positions on the bone are considered as references for the later processing in the STA assessment. All the steps are explained in details in the next subsections.

\subsubsection{Curve Fitting}

Next step is generating smooth curves which pass through the key data points of the ring formation of the motion capture data. To this end, we use a piecewise polynomial cubic spline interpolation. In piecewise polynomial cubic spline interpolation, a cubic 
polynomial is fitted between each pair of markers data of the ring formation to create a smooth curve. If we consider the markers data of motion capture, eight markers per each ring formation, are the sampled points from our desired curve, our goal is to find an approximated function between each consecutive pair of these eight points. For one dimension of the points, we have 8 distinct nodes $x_{i}$ such that: $x_{1}, x_{2}, \ldots, x_{7}, x_{8}$. Equation (16) gives the cubic polynomial in each subinterval to have a closed interpolated curve.

$$
S(x)= \begin{cases}S_{1}(x), & x \in\left[x_{1}, x_{2}\right] \\ S_{2}(x), & x \in\left[x_{2}, x_{3}\right] \\ & \vdots \\ S_{7}(x), & x \in\left[x_{7}, x_{8}\right] \\ S_{8}(x), & x \in\left[x_{8}, x_{1}\right]\end{cases}
$$

Where $S_{i}(x)$, as given by Equation (17), is a cubic polynomial that will be used on the subintervals.

$$
S_{i}(x)=a_{i} x^{3}+b_{i} x^{2}+c_{i} x+d_{i}, i=1, \ldots, 7,8
$$

In order to define the spline, $S(x)$, four unknown parameters of each $S_{i}(x)$ should be found based on the interpolation conditions and continuity conditions in both the first and second derivatives that are expressed in Equation (18) and (19) for $i=2, \ldots, 7,8$.

$$
\begin{aligned}
& S_{i-1}\left(x_{i}\right)=S_{i}\left(x_{i}\right) \\
& S_{i-1}^{\prime}\left(x_{i}\right)=S_{i}^{\prime}\left(x_{i}\right) \\
& S_{i-1}^{\prime \prime}\left(x_{i}\right)=S_{i}^{\prime \prime}\left(x_{i}\right)
\end{aligned}
$$

To have a closed curve, the cubic polynomial in subintervals $\left[x_{1}, x_{2}\right]$ and $\left[x_{8}, x_{1}\right], S_{1}(x)$ and $S_{8}(x)$ should satisfy the following conditions in Equation (19).

$$
\begin{aligned}
& S_{8}\left(x_{1}\right)=S_{1}\left(x_{1}\right) \\
& S_{8}^{\prime}\left(x_{1}\right)=S_{1}^{\prime}\left(x_{1}\right) \\
& S_{8}^{\prime \prime}\left(x_{1}\right)=S_{1}^{\prime \prime}\left(x_{1}\right)
\end{aligned}
$$

By applying the conditions, the cubic polynomials in the subintervals and consequently $S(x)$ are determined. 
As cubic spline interpolation is continuous in both the first and second derivatives everywhere in subintervals and at the merging points, it is a useful interpolating method in our application to produce smooth interpolated functions.

\subsubsection{Define a Plane through the Curves}

In order to determine the bone position at the three key markers, we need to define a plane containing the bone that passes through each curve from the previous step. To define the plane, we need to provide three non-collinear points on the plane: one of the three key markers, $P$, one data point on the curve that is very close to the marker, $Q$, and one other marker data on the opposite side of the first marker data, $R$. Figure 4.9 shows these three points.

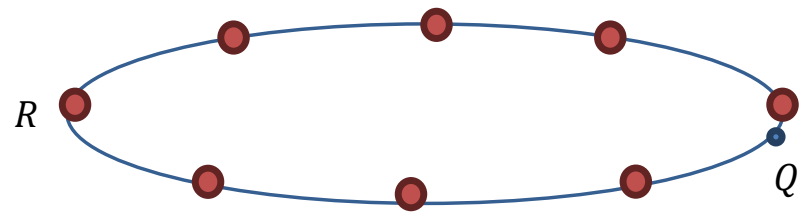

Skin Markers

- Point on the Curve

- Curve Fitted to the Markers

Figure 4.9 - Passing a Plane through Each Curve

The general equation of a plane is defined by Equation (20).

$$
a x+b y+c z+d=0
$$

Given the coordinates of these three points in space, $P=\left[x_{P}, y_{P}, z_{P}\right], Q=\left[x_{Q}, y_{Q}, z_{Q}\right]$, and $R=\left[x_{R}, y_{R}, z_{R}\right]$, we can find the parameters of the equation of the plane using the following equations:

$$
\begin{gathered}
a=y_{P}\left(z_{Q}-z_{R}\right)+y_{Q}\left(z_{R}-z_{P}\right)+y_{R}\left(z_{P}-z_{Q}\right) \\
b=z_{P}\left(x_{Q}-x_{R}\right)+z_{Q}\left(x_{R}-x_{P}\right)+z_{R}\left(x_{P}-x_{Q}\right) \\
c=x_{P}\left(y_{Q}-y_{R}\right)+x_{Q}\left(y_{R}-y_{P}\right)+x_{R}\left(y_{P}-y_{Q}\right) \\
d=-x_{P}\left(y_{Q} z_{R}-y_{R} z_{Q}\right)-x_{Q}\left(y_{R} z_{P}-y_{P} z_{R}\right)-x_{R}\left(y_{P} z_{Q}-y_{Q} z_{P}\right)
\end{gathered}
$$




\subsubsection{Bone Position at Three Key Markers Positions}

Once the plane has been defined, we apply the ultrasound depth measurements at the positions of three key markers to determine three points on the bone. In order to determine these points on the bone, they should satisfy three conditions:

- This point should lie on the plane from the previous step.

- The distance between the bone position and the key marker data on the position that the ultrasound depth is measured should be equal to the ultrasound depth measurement.

- If we define two vectors, one between the key marker data and the data point on the curve which is very close to the marker and the other vector between the key marker data and the bone point, these two vectors should be perpendicular; as the UDM is the minimal distance between the skin surface and the bone.

Figure 4.10 illustrates the curve fitted to the marker data and a point on the underlying bone at the position of one of the key markers.

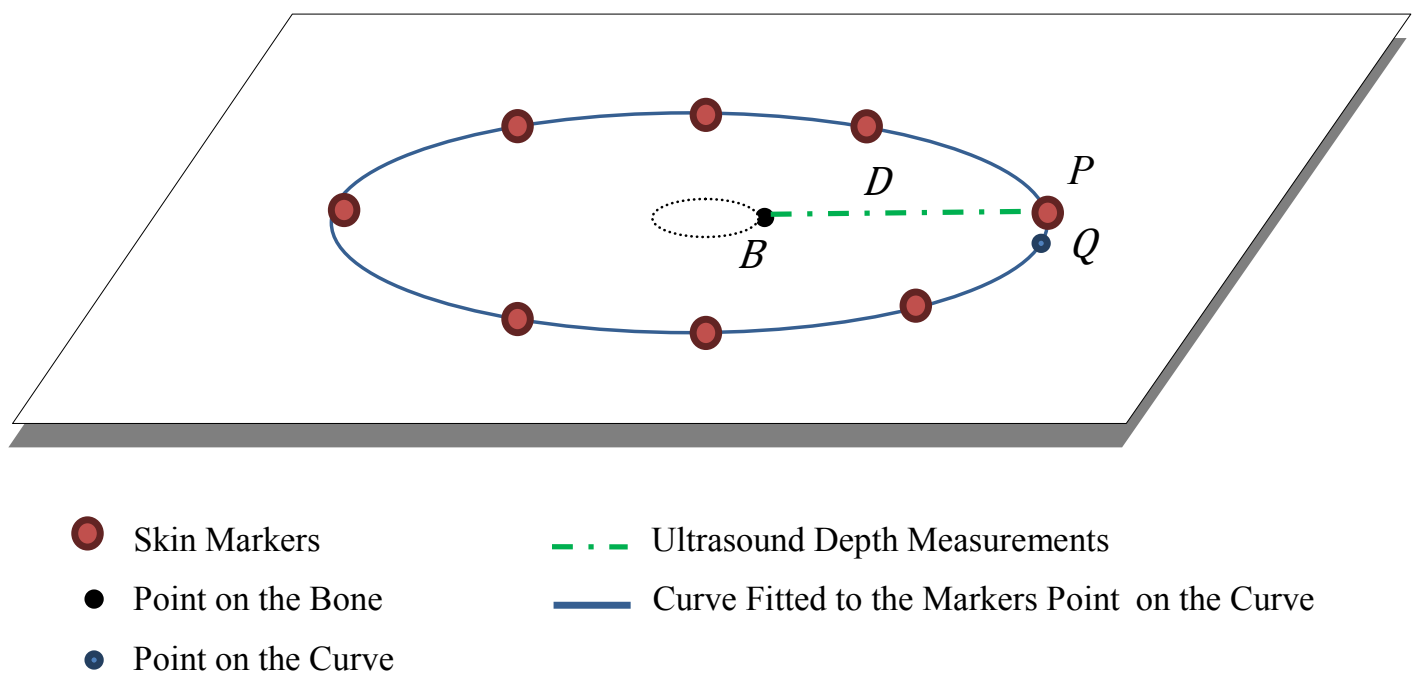

Figure 4.10 - Determining Points on the Bone

The conditions for the points on the femur bone can be written as Equations 22, 23 and 24 , respectively. In the following equations, $B=\left[x_{B}, y_{B}, z_{B}\right]$ is the desired point on the 
bone and $D$ is the ultrasound depth measurement. The coordinate of the bone point satisfies Equation (22) in order to have it on the plane passing through the key marker point.

$$
a x_{B}+b y_{B}+c z_{B}+d=0
$$

The distance between the bone position and the key marker which is equal to the ultrasound depth measurement is given by Equation (23).

$$
D=\sqrt{\left(x_{B}-x_{P}\right)^{2}+\left(y_{B}-y_{p}\right)^{2}+\left(z_{B}-z_{P}\right)^{2}}
$$

Vector $P Q$ and $P B$ are perpendicular if the dot product is equal to zero as given by Equation (24).

$$
\begin{gathered}
P Q . P B=0 \\
P Q=\left[x_{Q}-x_{P}, y_{Q}-y_{p}, z_{Q}-z_{P}\right] \text { and } P B=\left[x_{B}-x_{P}, y_{B}-y_{p}, z_{B}-z_{P}\right] \\
P Q . P B=\left(x_{Q}-x_{P}\right)\left(x_{B}-x_{P}\right)+\left(y_{Q}-y_{p}\right)\left(y_{B}-y_{p}\right)+\left(z_{Q}-z_{P}\right)\left(z_{B}-z_{P}\right) \\
\quad=0
\end{gathered}
$$

\subsubsection{Transformation of the Three Key Markers}

In the previous step, the bone positions at the three key markers of all movement types of the hip joint were determined. Determining these positions on the bone, the errors associated with the changes in tissue thickness at markers trajectories are eliminated; therefore these bone positions are considered as data without the STA. By having these points, we can find a rotation matrix and a translation vector that transform the bone positions at the three key markers of the standing position to each of the other movements. We derive the matrix and vector by solving a linear least square problem recursively. Our objective function for each movement (compared with standing position) is given by Equation (25). 


$$
\min _{\boldsymbol{R}, t} \sum_{i=1}^{3}\left\|\boldsymbol{R} s_{i}+t-m_{i}\right\|^{2}
$$

Where $\boldsymbol{R}$ is the rotation matrix $(3 \times 3), t$ is the translation vector $(3 \times 1), s_{i}$ is the vector of key marker $i$ in standing position $(3 \times 1)$, and $m_{i}$ is the corresponding key marker of the other movements $(3 \times 1)$.

\subsection{Quantification of Soft Tissue Artefact}

The most important aspect of STA is to determine how the markers are displaced relative to the underlying bone due to the movement. Due to muscle contractions and skin deformation, markers move during different range of motions of the hip. We proposed a method to determine three points on the bone that are the projection of positions of three markers having less change in their tissue thickness during all range of the hip movements. We proposed an approach to determine the transformation matrix and translation vector of the bone positions from the standing position to the other types of movements. Determining the transformation matrix and translation vector, we have the rigid movement of the bone. If there were no STA error in markers trajectories, they would move rigidly the same as the bone. Therefore in order to quantify STA, we apply the matrix and vector to the trajectories of the markers of standing position, compare with the trajectories of markers of the other movements, and compute the displacement of the markers.

\subsection{Determination of Hip Joint Centre}

In order to determine the HJC, we use the SCoRE algorithm [23]. The SCoRE technique considers both of the joint segments, femur and pelvis, in the CoR estimation. In this algorithm a local coordinate system for each moving segment of the joint (pelvis and femur head) is defined, and then these local systems for all time frames are transferred into a global reference system to estimate the HJC. In order to estimate the joint centre, the SCoRE technique minimizes the residual of the centres in the global coordinate system which is equivalent to the objective function given by Equation (26). 


$$
f_{\text {SCoRE }}\left(C_{F}, C_{P}\right)=\sum_{i=1}^{n}\left\|\boldsymbol{R}_{i} C_{F}+t_{i}-\left(\boldsymbol{S}_{i} C_{P}+d_{i}\right)\right\|^{2}
$$

Where $C_{F}, C_{P}$ are the joint centres of the femoral and pelvic segments in the local coordinate systems, $R_{i}, S_{i}$ are rotation matrices and $t_{i}, d_{i}$ are translation vectors to transform the local coordinate systems of the pelvis and femur to an appropriate global system. Equation (26) can be written as a linear least square problem which is given by Equation (27).

$$
\left(\begin{array}{cc}
\boldsymbol{R}_{1} & -\boldsymbol{S}_{1} \\
\vdots & \vdots \\
\boldsymbol{R}_{n} & -\boldsymbol{S}_{n}
\end{array}\right)\left(\begin{array}{c}
C_{F} \\
C_{P}
\end{array}\right)=\left(\begin{array}{c}
d_{1}-t_{1} \\
\vdots \\
d_{n}-t_{n}
\end{array}\right)
$$

In order to calculate the HJC using SCoRE algorithm, rotation matrices and translation vectors are needed to be determined. One method to calculate is unit vector which require three non-collinear markers on each segment, the pelvis and thigh. If we consider the trajectories of three markers placed on the femoral segment are $F_{1}, F_{2}$ and $F_{3}$, on the pelvic segment are $P_{1}, P_{2}$ and $P_{3}, \boldsymbol{R}=\left[R^{1} R^{2} R^{3}\right]$ and $\boldsymbol{S}=\left[S^{1} S^{2} S^{3}\right]$, the calculation of the rotation matrices of femoral segment and pelvic segment are as Equations (28) and (29), respectively.

$$
\begin{gathered}
R^{1}=\frac{\left(F_{1}-F_{2}\right)}{\left|F_{1}-F_{2}\right|} \\
R^{2}=\frac{\left(F_{3}-F_{2}\right) \times R^{1}}{\left|\left(F_{3}-F_{2}\right) \times R^{1}\right|} \\
R^{3}=R^{1} \times R^{2} \\
S^{1}=\frac{\left(P_{1}-P_{2}\right)}{\left|P_{1}-P_{2}\right|} \\
S^{2}=\frac{\left(P_{3}-P_{2}\right) \times S^{1}}{\left|\left(P_{3}-P_{2}\right) \times S^{1}\right|} \\
S^{3}=S^{1} \times S^{2}
\end{gathered}
$$


Where $(a-b)$ is a distance vector between two vectors $a$ and $b,|a|$ shows the norm of vector $a$, and $a \times b$ returns the cross product of vectors $a$ and $b$. Above equations give the rotation matrices of the femoral and pelvic segments, $\boldsymbol{R}=\left[R^{1} R^{2} R^{3}\right]$ and $\boldsymbol{S}=$ $\left[S^{1} S^{2} S^{3}\right]$, in the global system for all time frames. In order to use Equation (27), translation vectors are required which are calculated

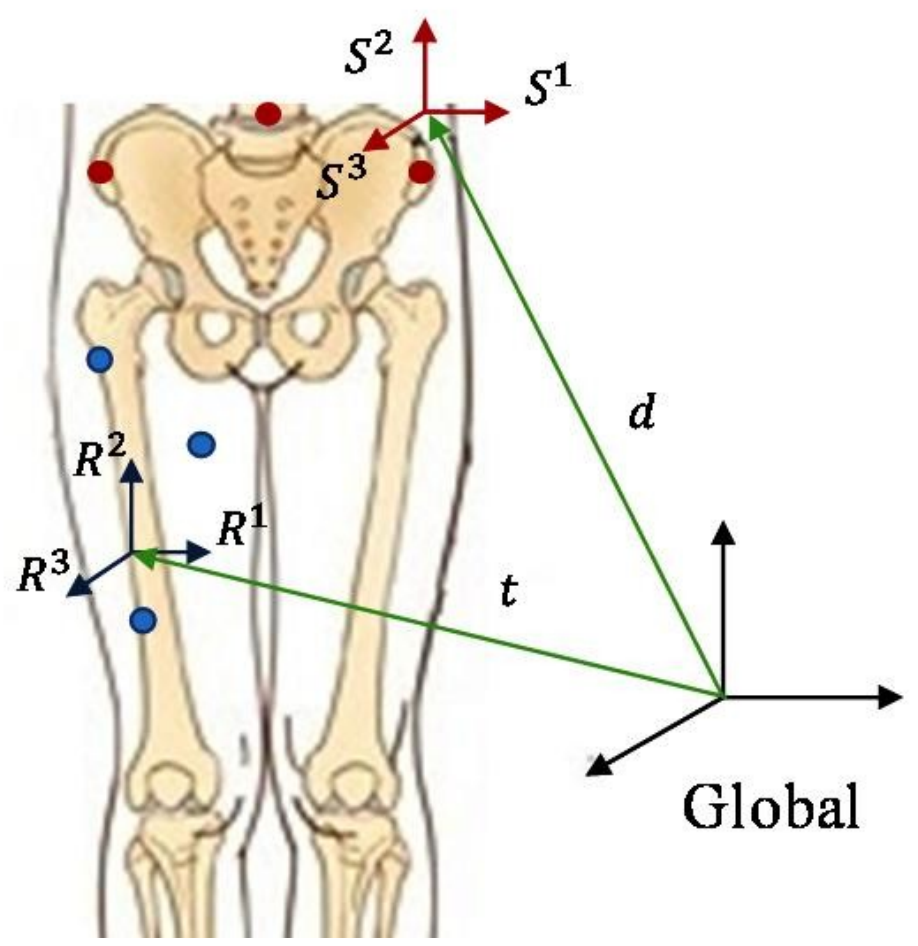

Figure 4.11 - Global and Local Coordinate Systems for the Femoral and Pelvic Segments

Since the SCoRE algorithm gives two centre positions, one for the pelvis and one for the femur head, its accuracy can be evaluated by the Euclidean distance between these two centres (after transforming in to a global reference system) which is known as the SCoRE residual [92]. SCoRE residual is given by Equation (30).

$$
r=\left|\boldsymbol{R}_{i} C_{F}+t_{i}-\left(\boldsymbol{S}_{i} C_{P}+d_{i}\right)\right|
$$

Where $r$ is SCoRE residual and $C_{F}, C_{P}$ are the joint centres of the femoral and pelvic segments. 


\section{Chapter 5: Experiments and Results}

\subsection{Subjects}

The acquisition was performed at Carleton University's Motion Capture Studio and Ultrasound Imaging Laboratory. The study was carried out on 10 volunteers ( 5 females and 5 males) aged between 21 and 30 years (Mean: 27.2 years; Std. dev.: 2.7 years) with a mean body weight of $64.1 \mathrm{Kg}$ (Std. Dev.: 13.9) and a mean height $172.1 \mathrm{~cm}$ (Std. Dev.: 8.7) as shown in Table 5.1. The volunteers were healthy with no functional problems of the lower limb. The study was approved by the Carleton Ethics Board and the volunteers gave written informed consent, as represented in appendices A, B, and C.

Table 5.1 - Subject's Age, Weight, Height, and Gender

\begin{tabular}{|c|c|c|c|c|}
\hline Subject & Age & Weight $(\mathrm{Kg})$ & Height $(\mathrm{cm})$ & Gender (F/M) \\
\hline$\# 1$ & 28 & 54 & 165 & $\mathrm{~F}$ \\
\hline$\# 2$ & 26 & 47 & 160 & $\mathrm{~F}$ \\
\hline$\# 3$ & 30 & 54 & 167 & $\mathrm{~F}$ \\
\hline$\# 4$ & 27 & 57 & 167 & $\mathrm{~F}$ \\
\hline$\# 5$ & 26 & 47 & 162 & $\mathrm{~F}$ \\
\hline$\# 6$ & 30 & 70 & 178 & $\mathrm{M}$ \\
\hline$\# 7$ & 21 & 72 & 178 & $\mathrm{M}$ \\
\hline
\end{tabular}




\begin{tabular}{|c|c|c|c|c|}
\hline Subject & Age & Weight $(\mathrm{Kg})$ & Height $(\mathrm{cm})$ & Gender (F/M) \\
\hline$\# 8$ & 27 & 81 & 183 & $\mathrm{M}$ \\
\hline$\# 9$ & 30 & 75 & 181 & $\mathrm{M}$ \\
\hline$\# 10$ & 27 & 84 & 180 & $\mathrm{M}$ \\
\hline
\end{tabular}

The experimental procedure for each subject consisted of two parts: optical motion capture and ultrasound depth measurements, which are described in the following sections.

\subsection{Optical Motion Capture}

Two sets of markers were attached to the skin of the subjects: The first set of markers consisted of 8 spherical reflective ones at palpable bony landmarks including three markers on the hip area, left and right anterior superior iliac spine and the lower spine, two on either side of the knee, medial and lateral femoral epicondyles, and two on either side of the ankle, medial and lateral malleolus, and one on greater trochanter. The other set consisted of skin surface markers in four ring formations. The rings were placed approximately $5 \mathrm{~cm}$ apart, with eight markers per ring. The markers configuration of the thigh is illustrated in Figure 5.1.

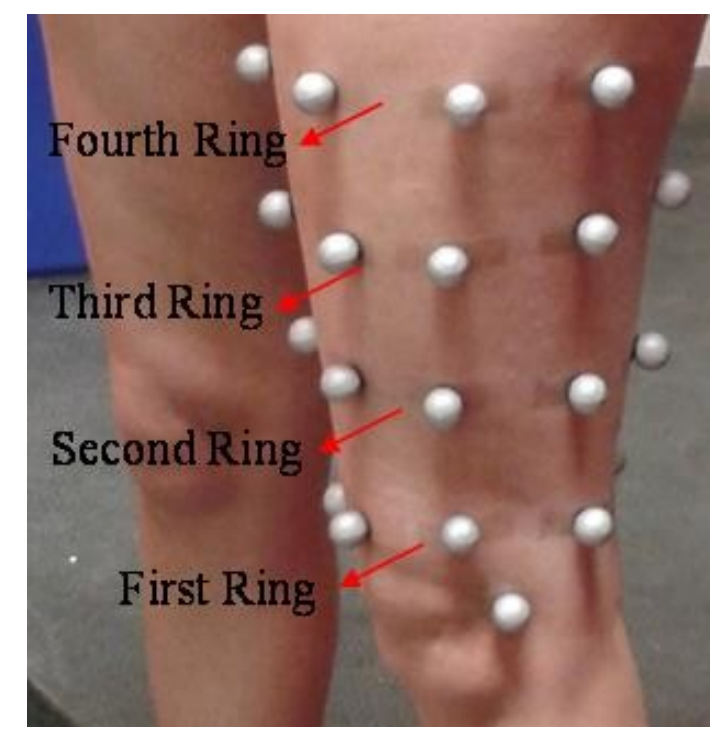

Figure 5.1- Subject's Thigh Markers Configuration 
Once the vicon system was calibrated and the markers were attached to the subject's skin, the trajectories of the markers were tracked during standing position, and three motions: flexion, extension, and abduction as shown in Figure 5.2; the error in the marker positions is considered to be less than $1 \mathrm{~mm}$.

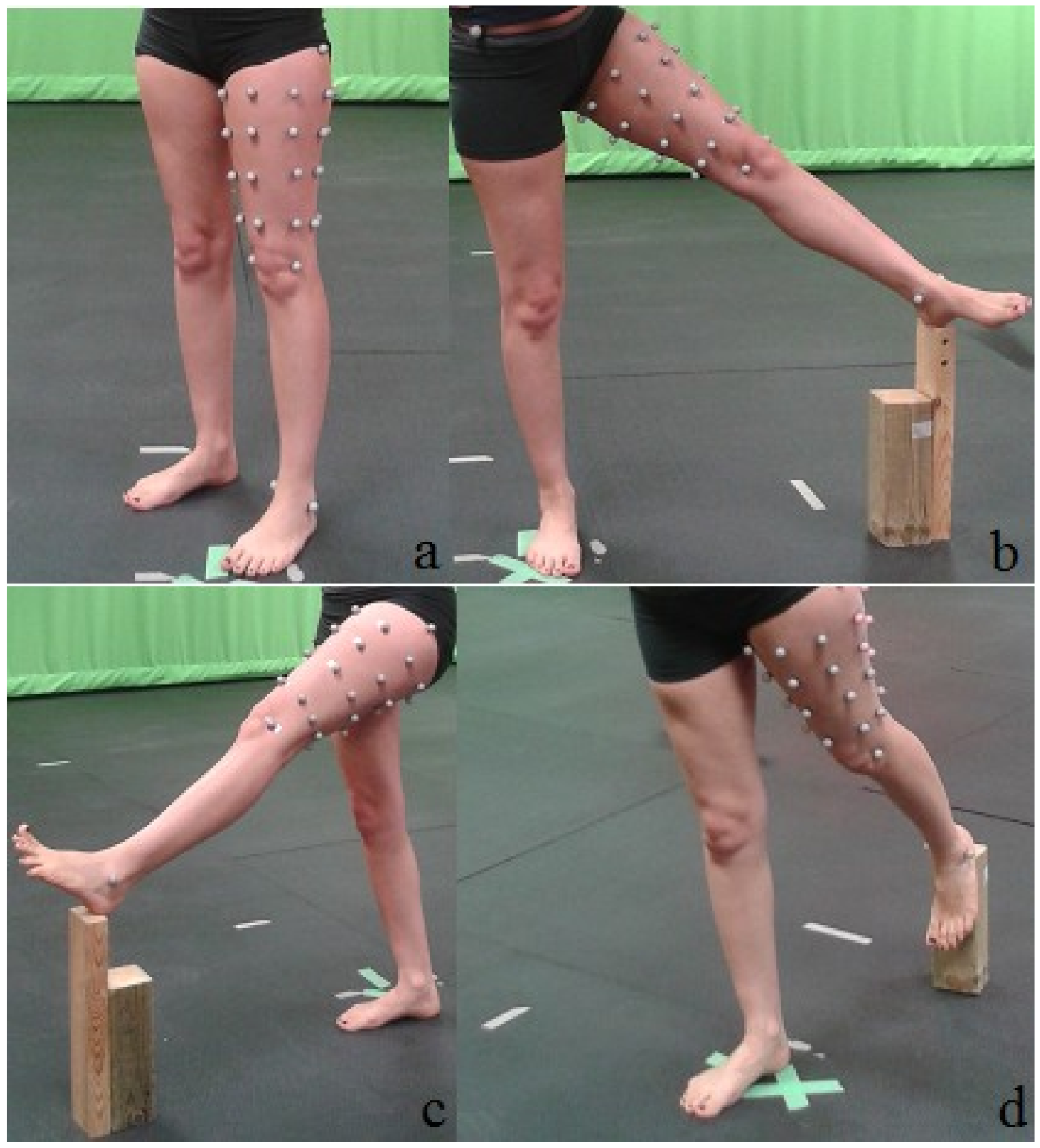

Figure 5.2 - Subject Positions during Optical Motion Capture, a) Standing, b) Abduction, c) Flexion, and d) Extension 
All the motions were started from standing position. In order to have the same range of motion of the hip joint for the ultrasound depth measurements, the positions were determined using non-reflective blocks that were setup ahead of capture with a specific configured distance as shown in Figure 5.2.

After tracking the markers trajectories for each subject, we processed optical motion capture data. In post-processing phase of data, the 3D positions of all markers (hip area and leg) during standing, flexion, extension, and abduction were reconstructed; then proper frames of the movements were selected, the markers were labeled and their trajectories were exported to a C3D file format to be used in MATLab for later processing. Figure 5.3 shows the 3D reconstruction of all markers positions of one of the subjects.

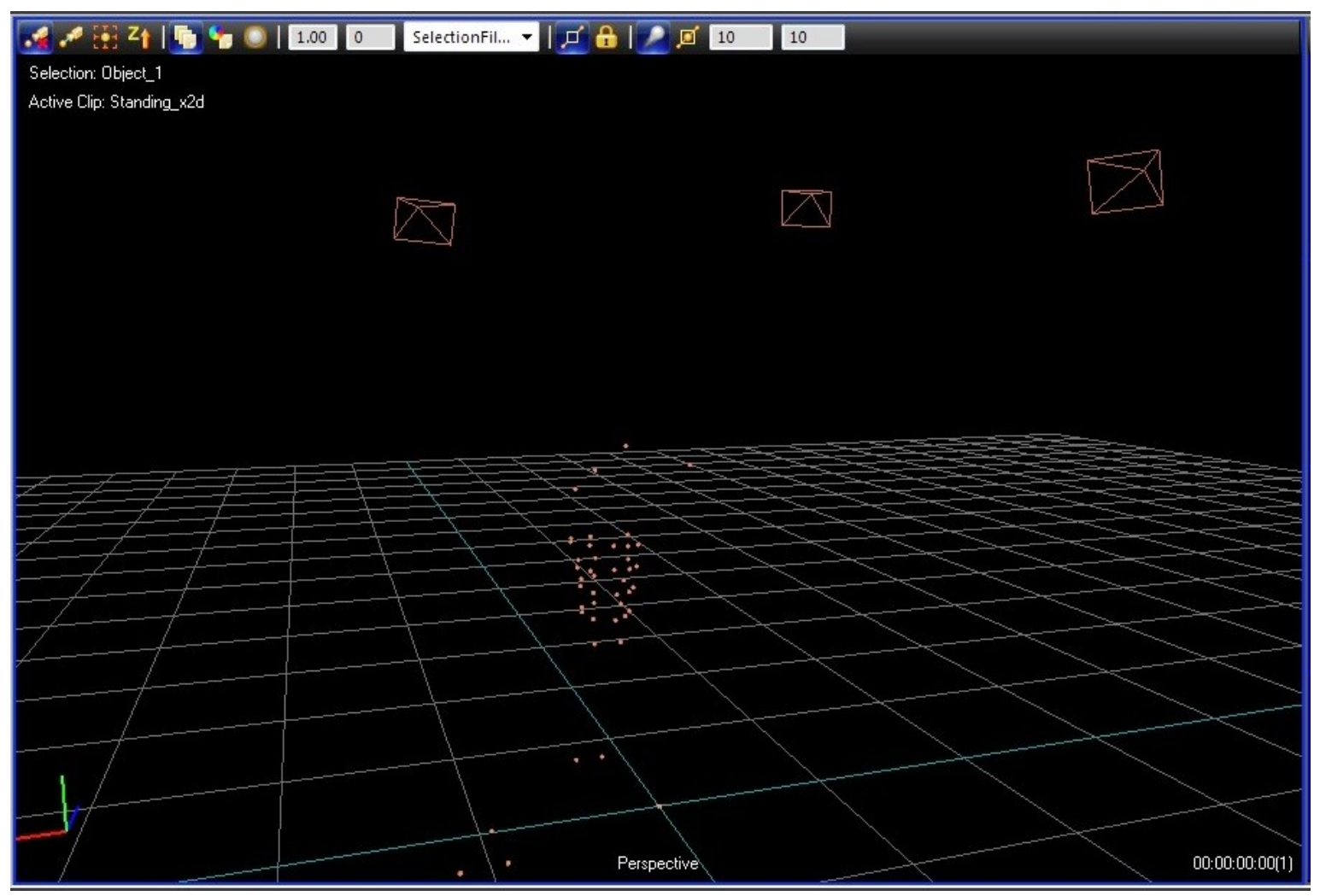

Figure 5.3 - 3D Reconstruction of Markers Positions of a Subject

The next experimental procedure was ultrasound depth measurements experiment which is combined with the information from motion capture to assess the STA. 


\subsection{Ultrasound Depth Measurements}

In ultrasound depth measurements experiment, the changes in the thickness of the soft tissue were measured at four locations of each ring during all movements, Standing, flexion, extension, and abduction. These four locations were at the same locations as the reflective markers placed on the skin surface during optical motion capture. Figure 5.4 shows one of the ring formations consisting of eight markers placed on the thigh. Changes in the tissue thickness were measured at the positions of markers A, C, E, and G which are located in the outer side, front side, inner side, and back side of the thigh, respectively.

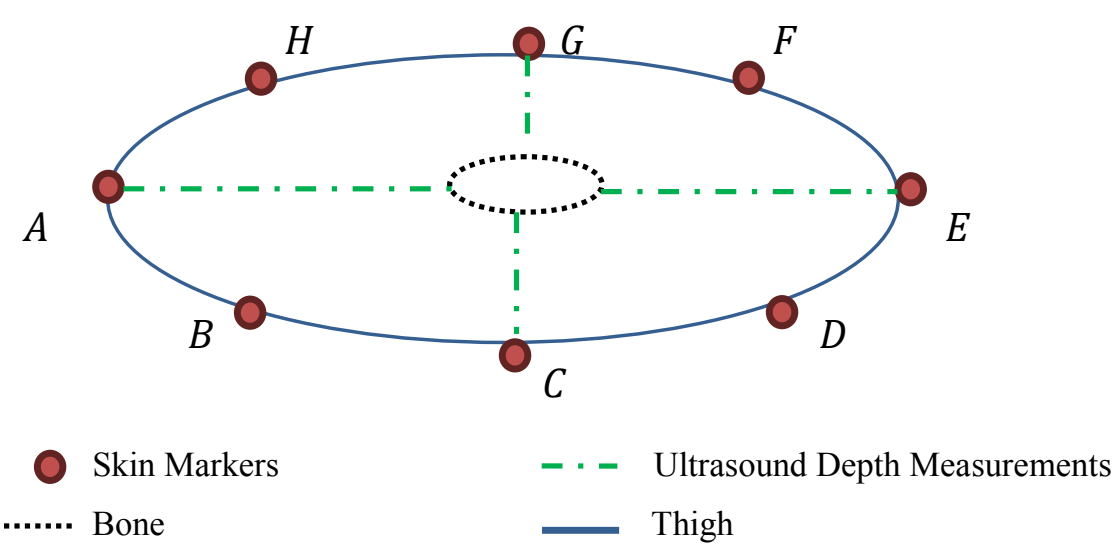

Figure 5.4 - Locations of Ultrasound Depth Measurements

During the ultrasound experiment, the subjects had the same range of motion of the hip as the optical motion capture using non-reflective blocks. Figure 5.5 shows the procedure of ultrasound depth measurements for one of the subjects at the front side of one ring. 


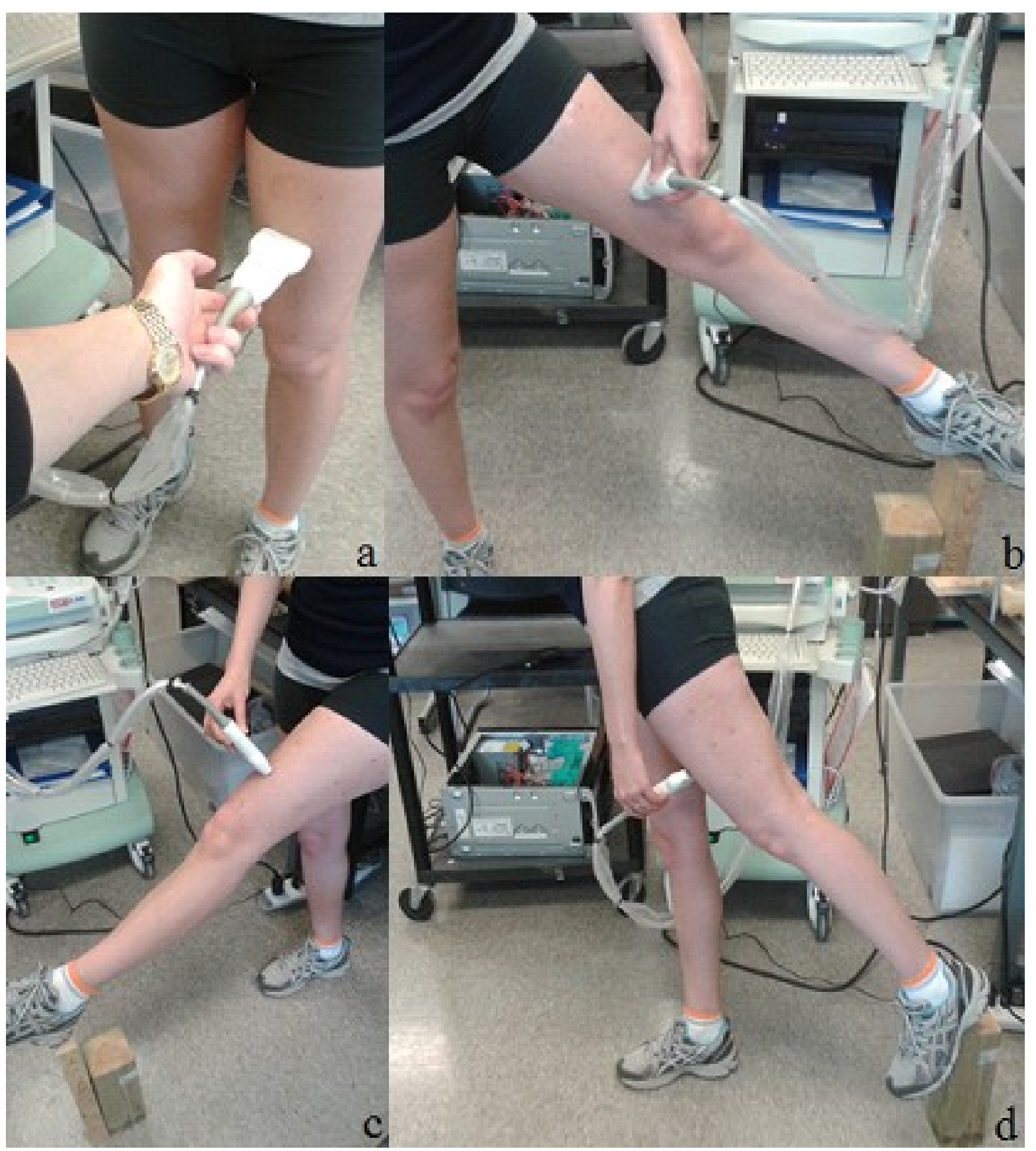

Figure 5.5 - Subject Positions during Ultrasound Depth Measurements, a) Standing, b) Abduction, c) Flexion, and d) Extension 
Figure 5.6 shows the tissue thickness at one marker location. As can be seen, this image is very noisy and consists of some high intensity pixels in different levels of depth. One of them corresponds to the surface bone and the others belong to the reflecting echoes from the layered structures of different muscles. These unrelated surfaces were distinguished by pushing the ultrasound probe before extracting the exact measurement of the soft tissue thickness. By pushing the ultrasound probe the pixels correspond to the bone surface were not changed. After detecting the desired edge, the probe was released to measure the real thickness of soft tissues.

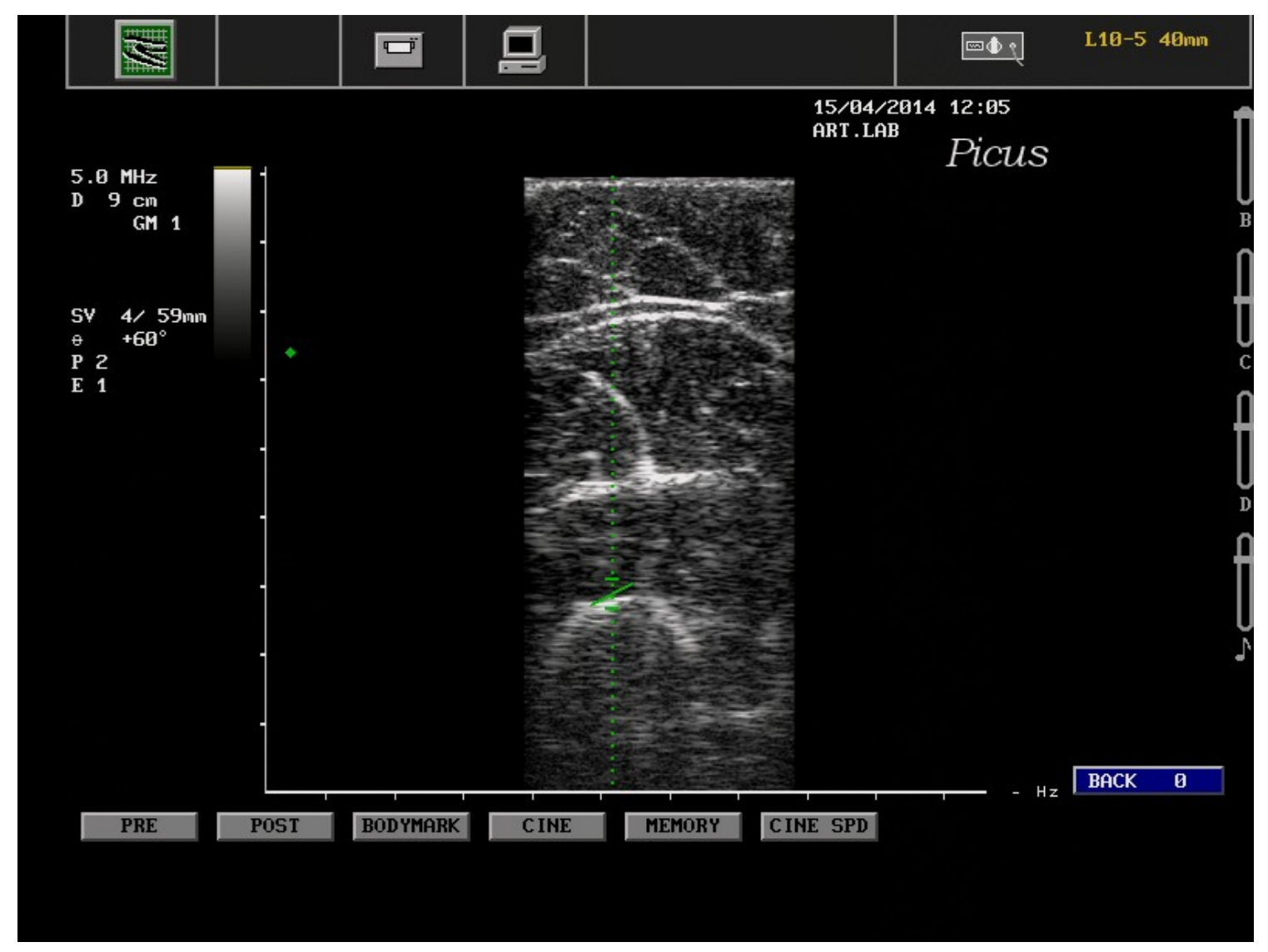

Figure 5.6 - Ultrasound Depth Measurement at a Marker Position, Standing Position

Figures 5.7, 5.8 and 5.9 represent the ultrasound depth measurements during flexion, extension and abduction at the same marker location as Figure 5.6. 


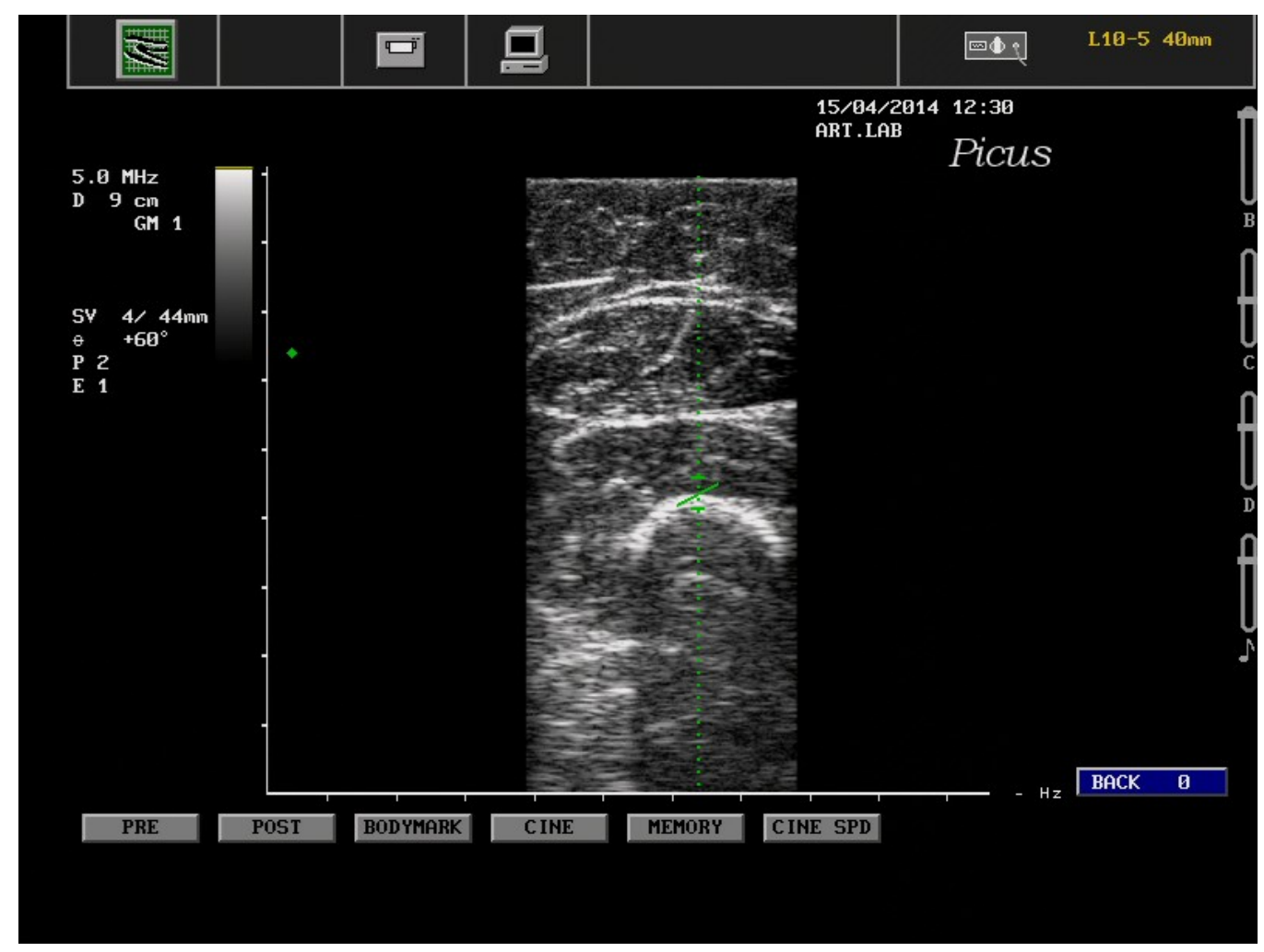

Figure 5.7 - Ultrasound Depth Measurement at a Marker Position, Flexion

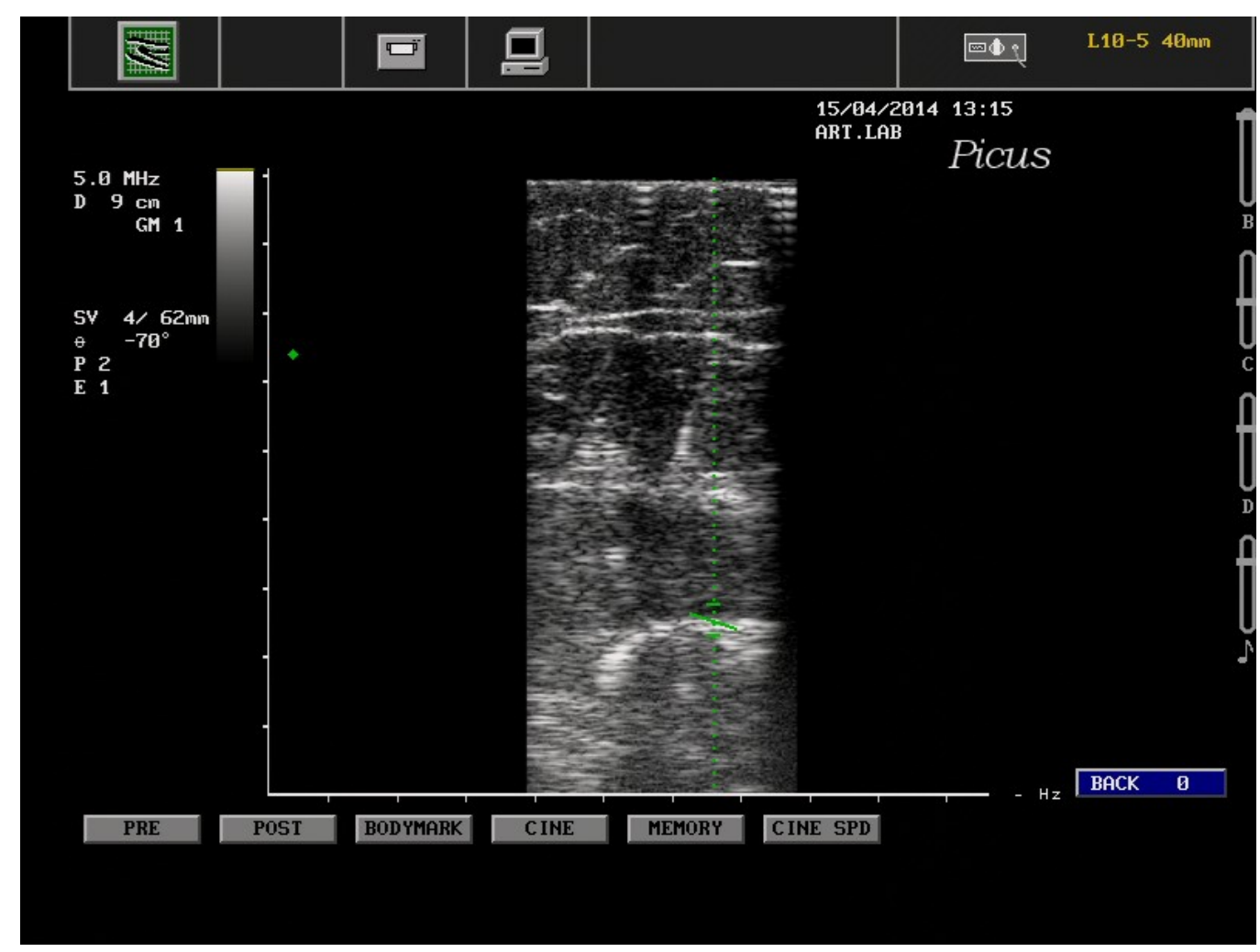

Figure 5.8 - Ultrasound Depth Measurement at a Marker Position, Extension 


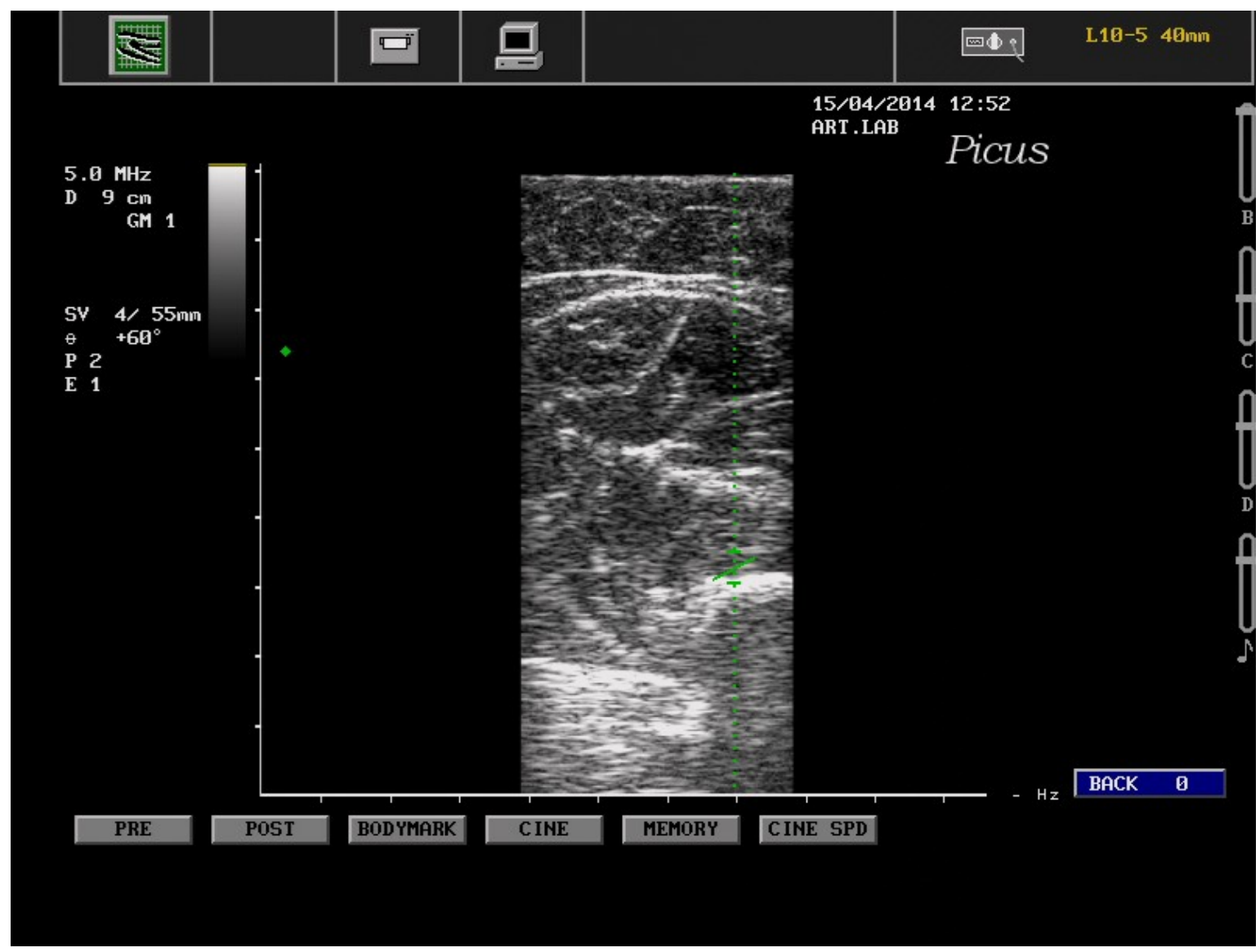

Figure 5.9 - Ultrasound Depth Measurement at a Marker Position, Abduction

As can be seen, the thickness of the tissue at this specific location is different during several movements of the hip. This thickness is $59 \mathrm{~mm}$ when the subject was in the standing position, $44 \mathrm{~mm}$ for flexion, $62 \mathrm{~mm}$ for extension, and $55 \mathrm{~mm}$ for abduction movement. This information is used for later processing. Table 5.2 illustrates all ultrasound depth measurements of one of the subjects. 
Table 5.2 - Ultrasound Depth Measurements for one of the Participants

\begin{tabular}{|c|c|c|c|c|c|}
\hline \multirow{2}{*}{\multicolumn{2}{|c|}{ Markers Positions \& Movement Types }} & \multicolumn{4}{|c|}{ Tissue Thickness Measurements (mm) } \\
\hline & & Front & Side & Back & Inside \\
\hline \multirow{4}{*}{ First Ring } & Standing & 31 & 34 & 60 & 38 \\
\hline & Flexion & 23 & 31 & 62 & 32 \\
\hline & Abduction & 30 & 26 & 45 & 52 \\
\hline & Extension & 35 & 29 & 56 & 34 \\
\hline \multirow{4}{*}{ Second Ring } & Standing & 46 & 41 & 67 & 59 \\
\hline & Flexion & 30 & 43 & 76 & 43 \\
\hline & Abduction & 44 & 37 & 62 & 74 \\
\hline & Extension & 47 & 38 & 59 & 54 \\
\hline \multirow{4}{*}{ Third Ring } & Standing & 55 & 45 & 71 & 73 \\
\hline & Flexion & 37 & 47 & 79 & 49 \\
\hline & Abduction & 52 & 41 & 79 & 80 \\
\hline & Extension & 57 & 43 & 65 & 77 \\
\hline \multirow{4}{*}{ Fourth Ring } & Standing & 59 & 46 & 90 & 78 \\
\hline & Flexion & 44 & 47 & 90 & 60 \\
\hline & Abduction & 55 & 41 & 85 & 86 \\
\hline & Extension & 62 & 45 & 76 & 86 \\
\hline
\end{tabular}


Once the tissue thicknesses were measured, three marker locations were determined based on their coefficient of variation. Each marker location had four ultrasound depth measurements corresponding standing position, flexion, extension, and abduction. The coefficient of variations of these four measurements at each marker location was calculated. Table 5.3 gives the coefficient of variation of ultrasound Depth measurements at each Marker location during all Movements.

Table 5.3 - Coefficient of Variation of Ultrasound Depth Measurements at each Marker Location during all Movements of one Subject

\begin{tabular}{|c|c|c|c|c|}
\hline \multirow{2}{*}{ Markers Positions } & \multicolumn{4}{|c|}{ Coefficient of Variation (CV) } \\
\cline { 2 - 5 } & Front & Side & Back & Inside \\
\hline First Ring & 0.16778 & 0.112217 & 0.136114 & 0.231244 \\
\hline Second Ring & 0.18998 & 0.05868 & 0.112707 & 0.223846 \\
\hline Third Ring & 0.18048 & 0.08947 & 0.09261 & 0.202543 \\
\hline Fourth Ring & 0.143164 & 0.05877 & 0.07744 & 0.158207 \\
\hline
\end{tabular}

The gray cells show the locations having the smallest coefficient of variation. These three locations are considered as three key markers and their ultrasound depth measurements will be used in the further processing. 


\subsection{Data Analysis}

The next step is analysis of motion capture data using the information from ultrasound depth measurements. By processing the motion capture data using MATLab and the MATLab curve-fitting toolbox, we were able to fit curves to the markers data and determine bone positions at three key markers from the previous step.

Figure 5.10 illustrates the trajectories of all markers placed on the skin surface of the subject during standing position in optical motion capture, the curves fitted to motion capture data, secondary points on the curves used to determine points on the bone, and the trajectories of key markers projections on the underlying bone.

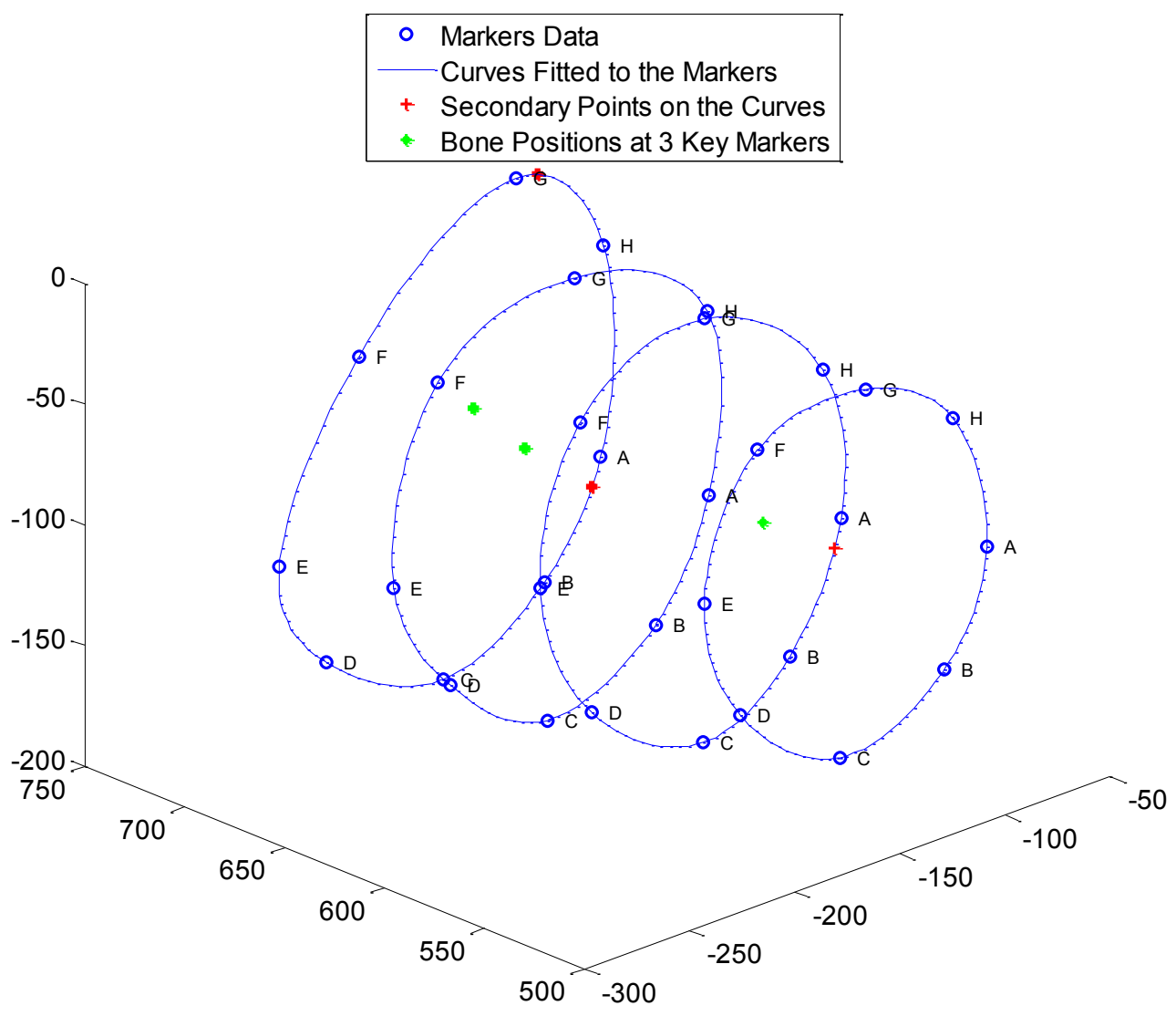

Figure 5.10 - Curve Fitting to Motion Capture Data and Determination of Bone Positions at 3 Key Points Positions of Standing Position 
After determination of the bone positions at the three key markers, we used these locations as references and we found rotation matrices and translation vectors which transformed the bone positions at the three key markers of the standing position to each of the other movements, flexion, extension and abduction. We derived rotation matrices and translation vectors by solving linear least square problems recursively in MATLab. If the markers locations didn't suffer from soft tissue artefacts, they would have the same movement as the bone from standing to the other movement types. Based on this fact, we applied the rotation matrix and translation vector (corresponding to each movement) to the markers trajectories of standing position and compared with the trajectories of markers of that corresponding movement, and then computed the displacement of the markers.

Figure 5.11, 5.12, and 5.13 illustrate the trajectories of markers from optical motion capture that have STA, and the corresponding trajectories after applying the rotation matrix and translation vector to the markers trajectories of standing position to have data without STA effects. These figures show the 3D displacements of the markers during flexion, extension and abduction.

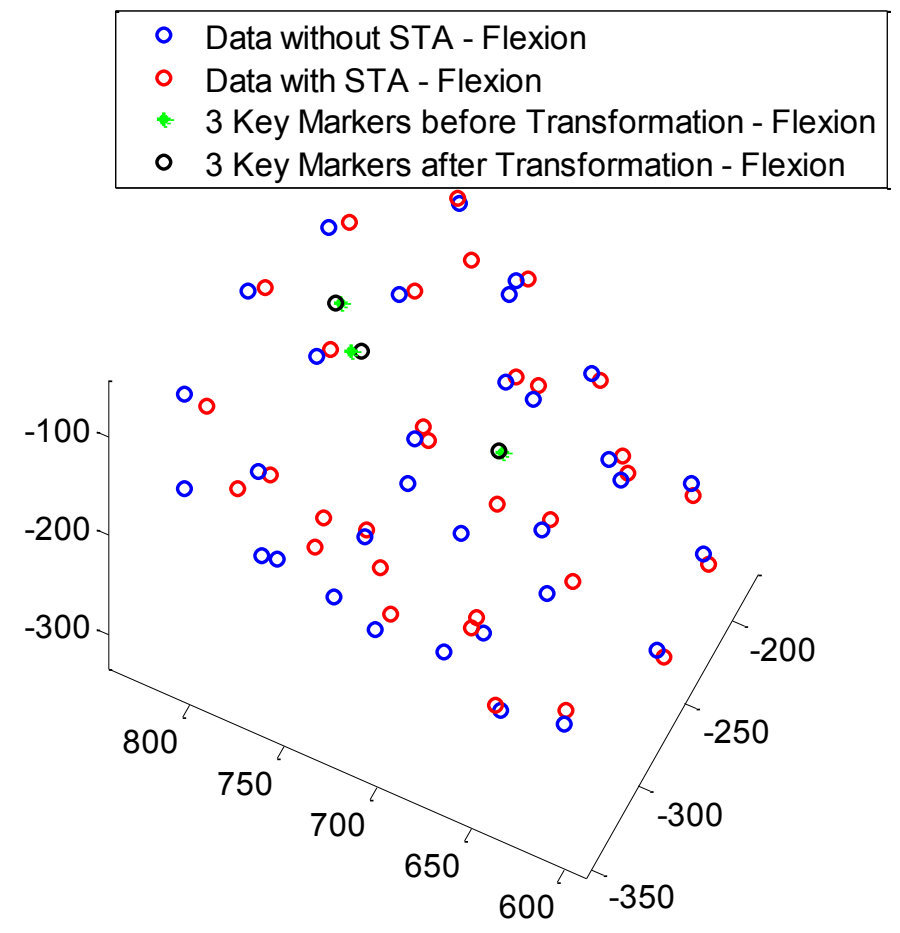

Figure 5.11 - Transformation of Standing Markers to Flexion Movement 


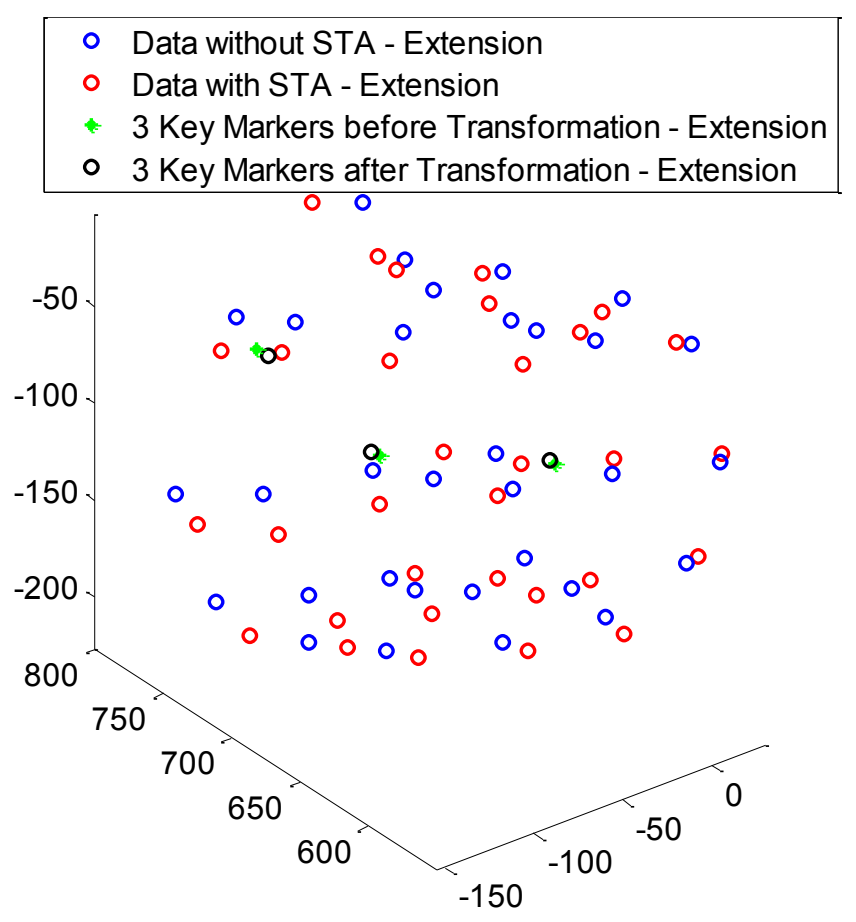

Figure 5.12 - Transformation of Standing Markers to Extension Movement

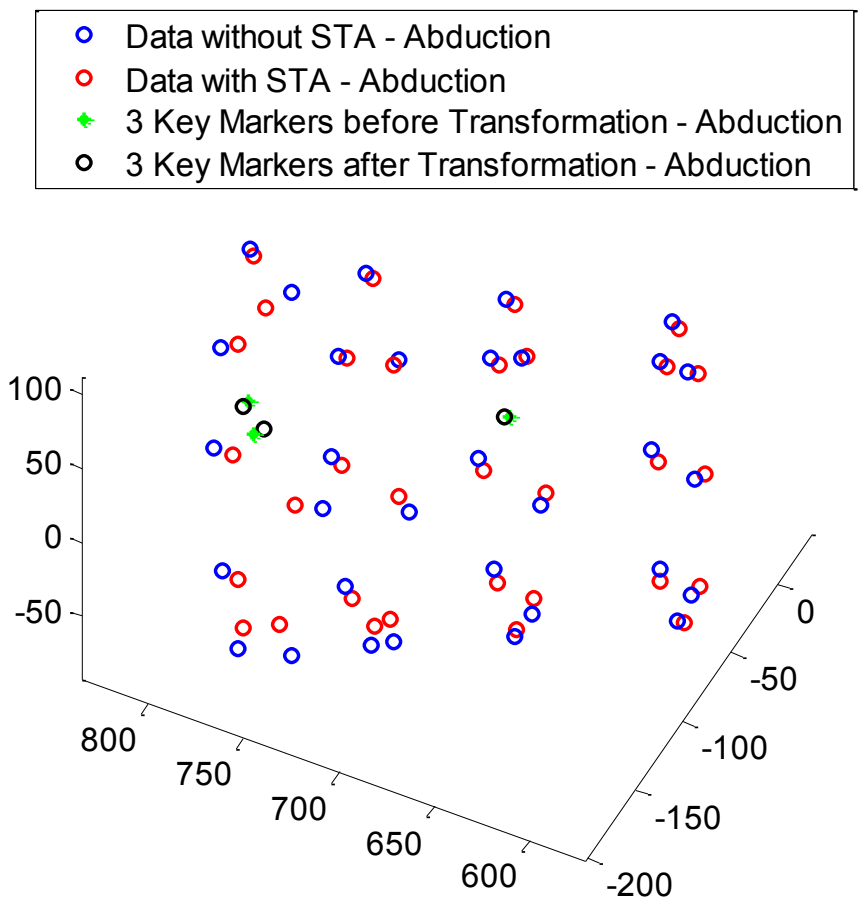

Figure 5.13 - Transformation of Standing Markers to Abduction Movement 
Then we calculated the displacements of all markers during flexion, extension and abduction movement. Figure 5.14, 5.15, and 5.16 show these calculations for one of the participants.

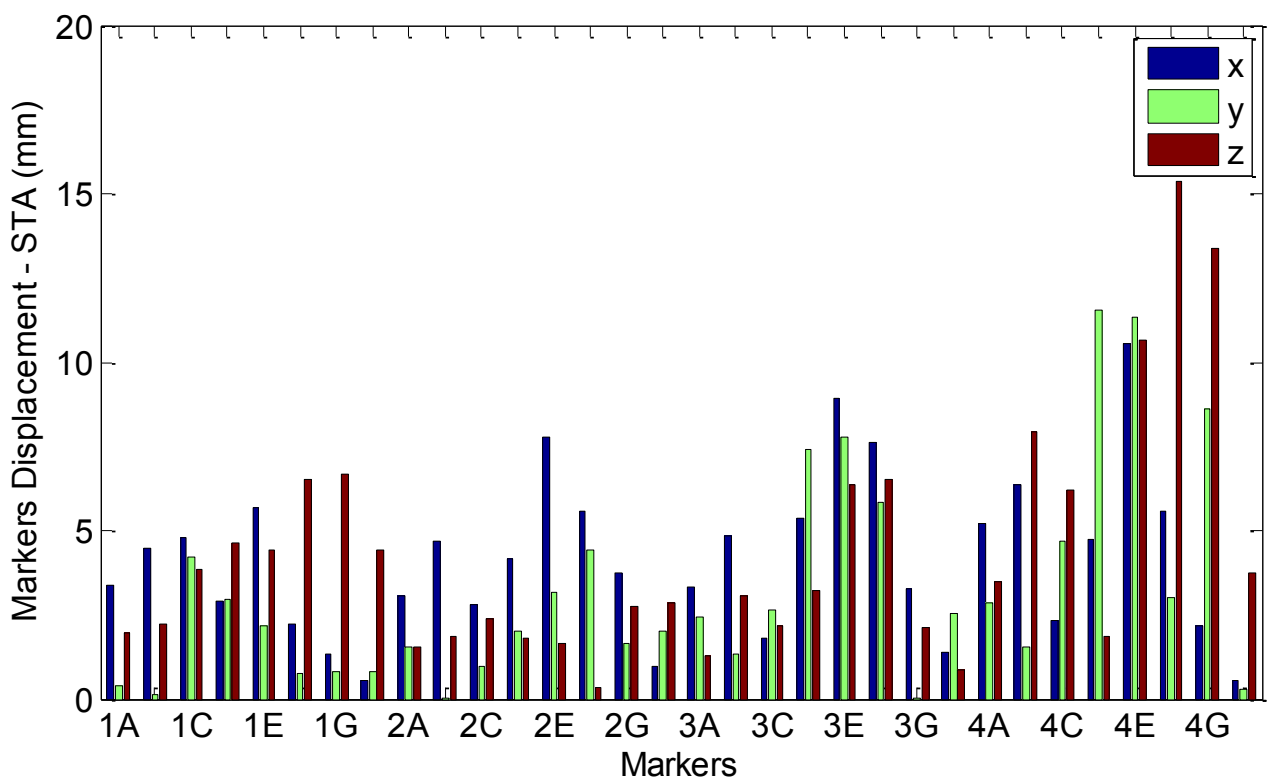

Figure 5.14 - Displacement of Markers (magnitude), Flexion

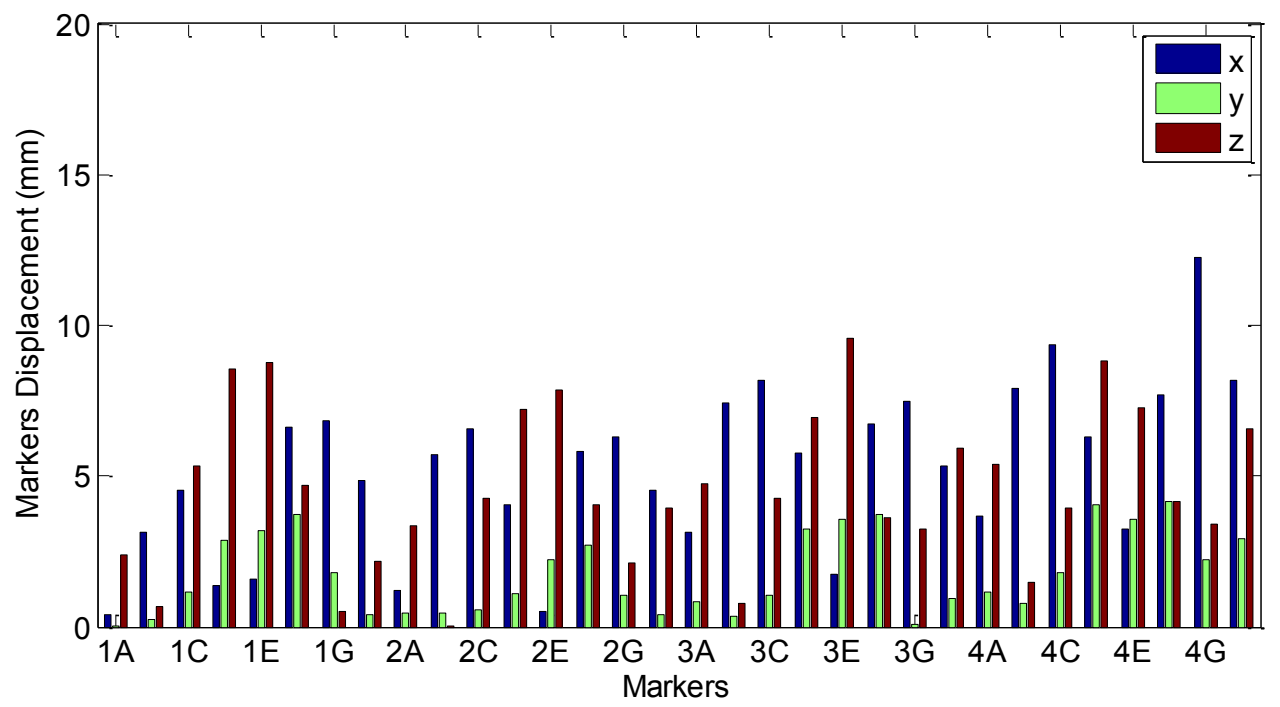

Figure 5.15 - Displacement of Markers (magnitude), Extension 


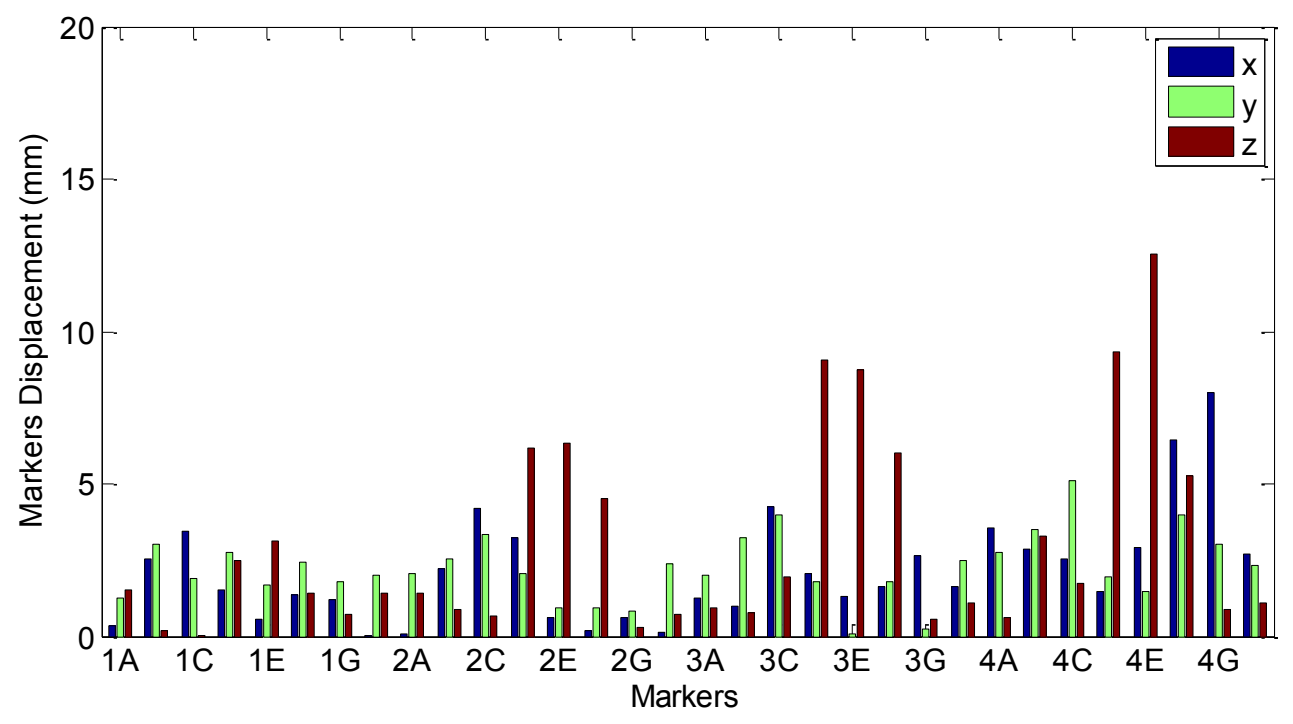

Figure 5.16 - Displacement of Markers (magnitude), Abduction

As the study was carried out on 10 subjects, in order to show the results of the markers displacements of all the subjects, we used box-plots. In the box-plot representation of markers displacements, the lowest value, highest value, median value, and the size of the first and third quartile of each marker displacement for all participants were illustrated as shown in Figure 5.17 to Figure 5.28. 


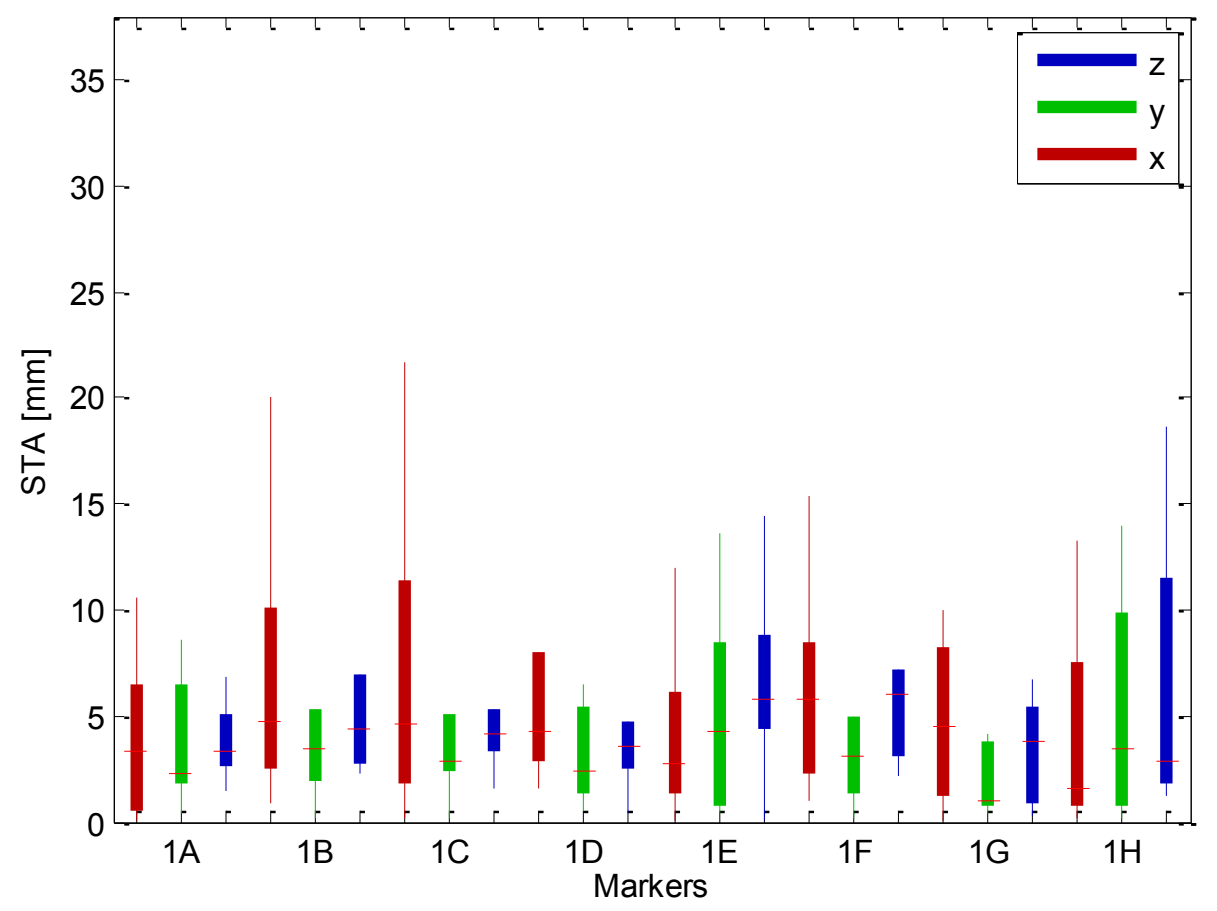

Figure 5.17 - Box-plots of STA Components of the First Ring of Markers Configuration during Flexion

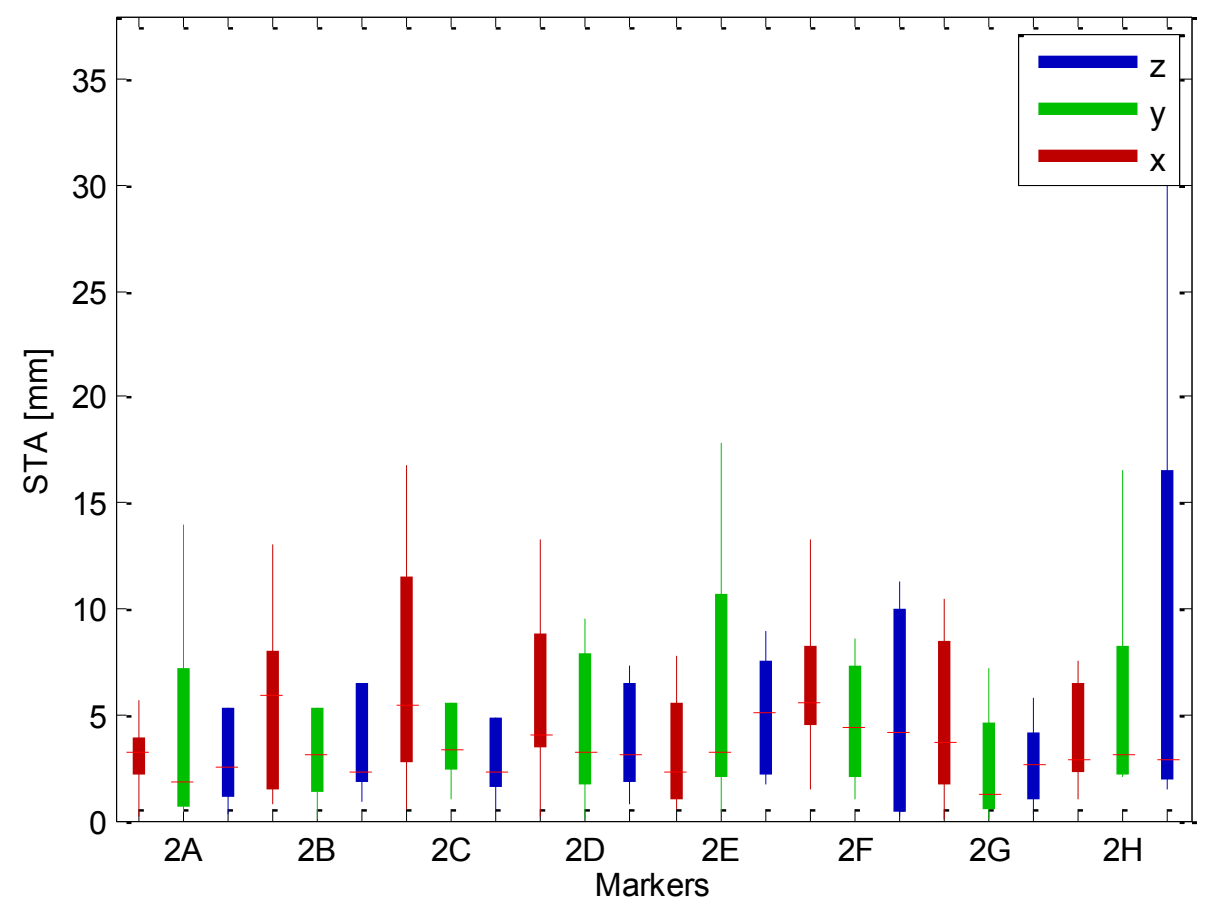

Figure 5.18 - Box-plots of STA Components of the Second Ring of Markers Configuration during Flexion 


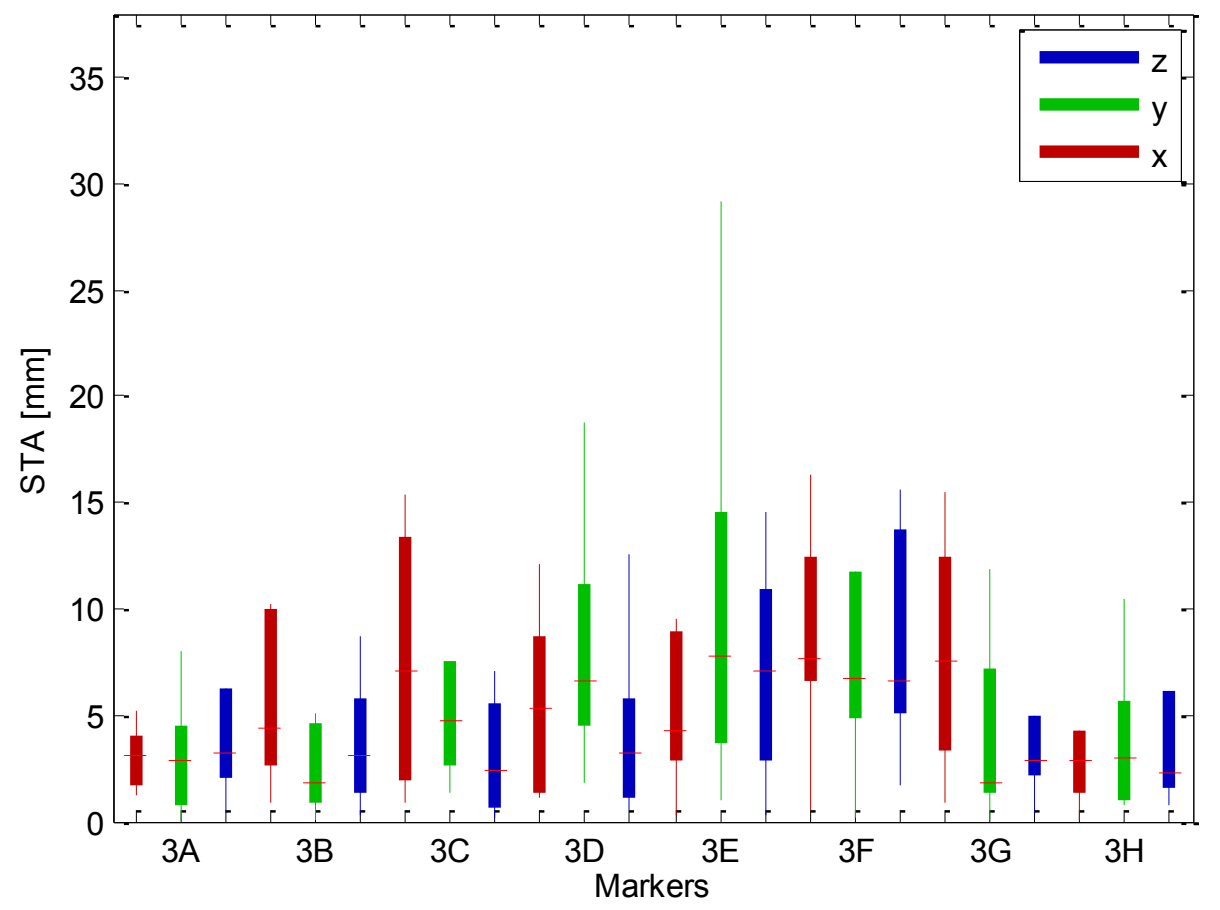

Figure 5.19 - Box-plots of STA Components of the Third Ring of Markers Configuration during Flexion

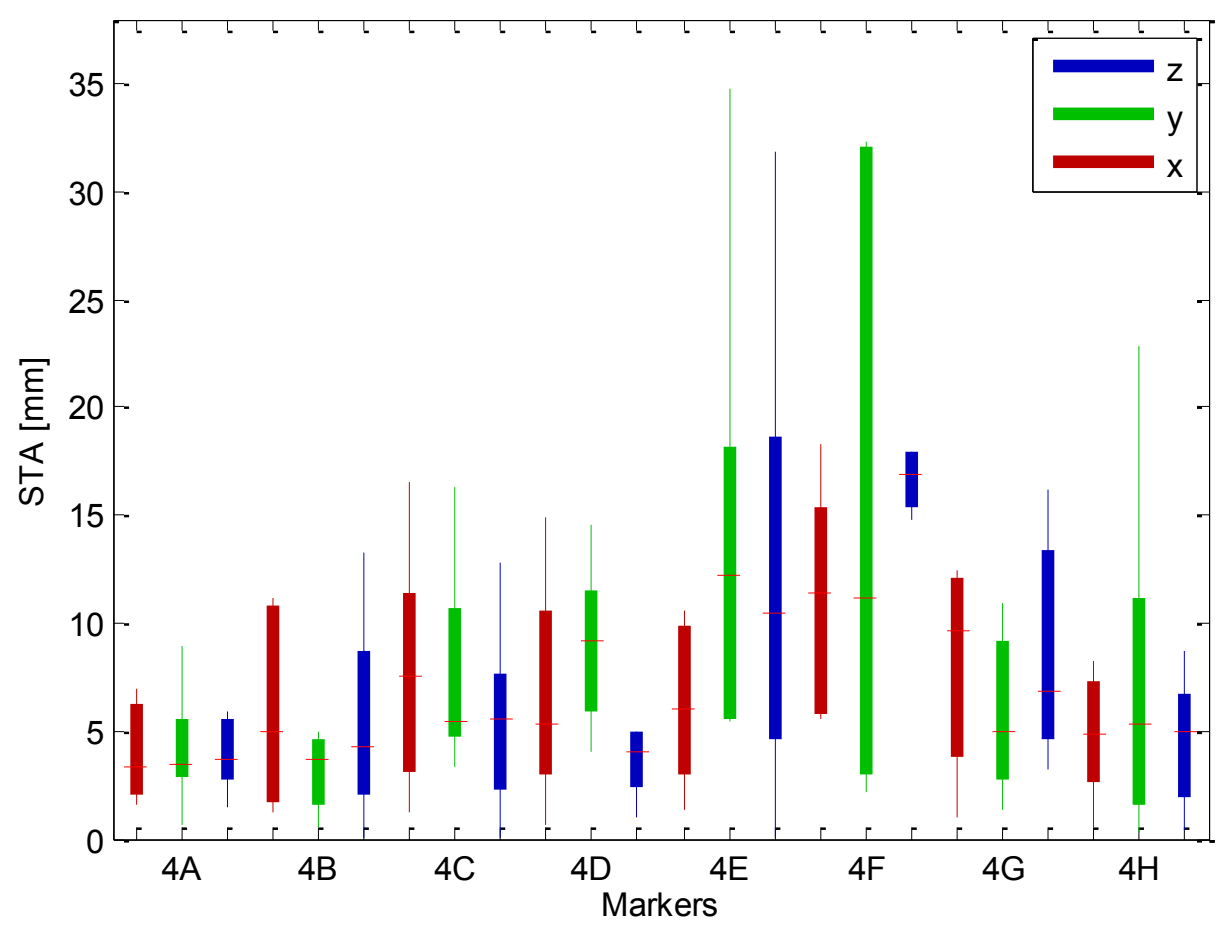

Figure 5.19 - Box-plots of STA Components of the Fourth Ring of Markers Configuration during Flexion 


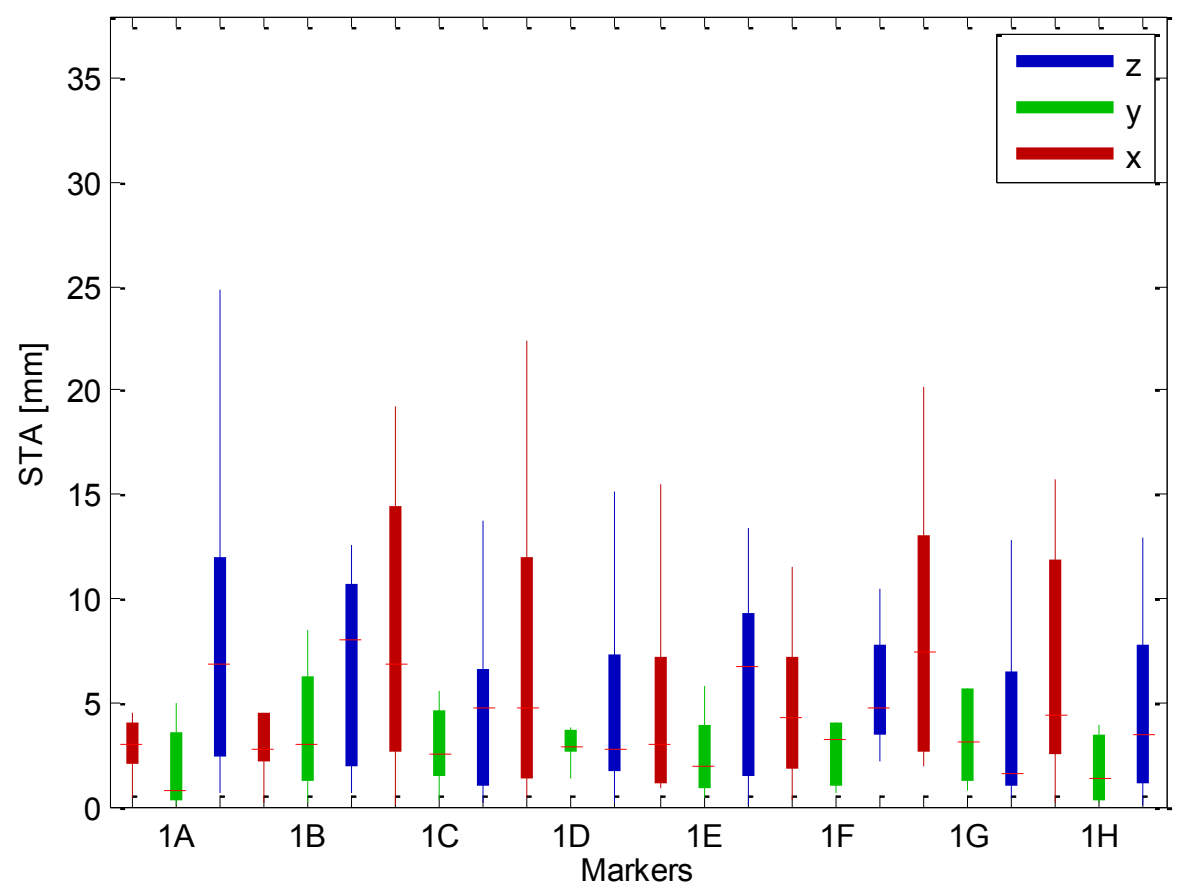

Figure 5.21 - Box-plots of STA Components of the First Ring of Markers Configuration during Extension

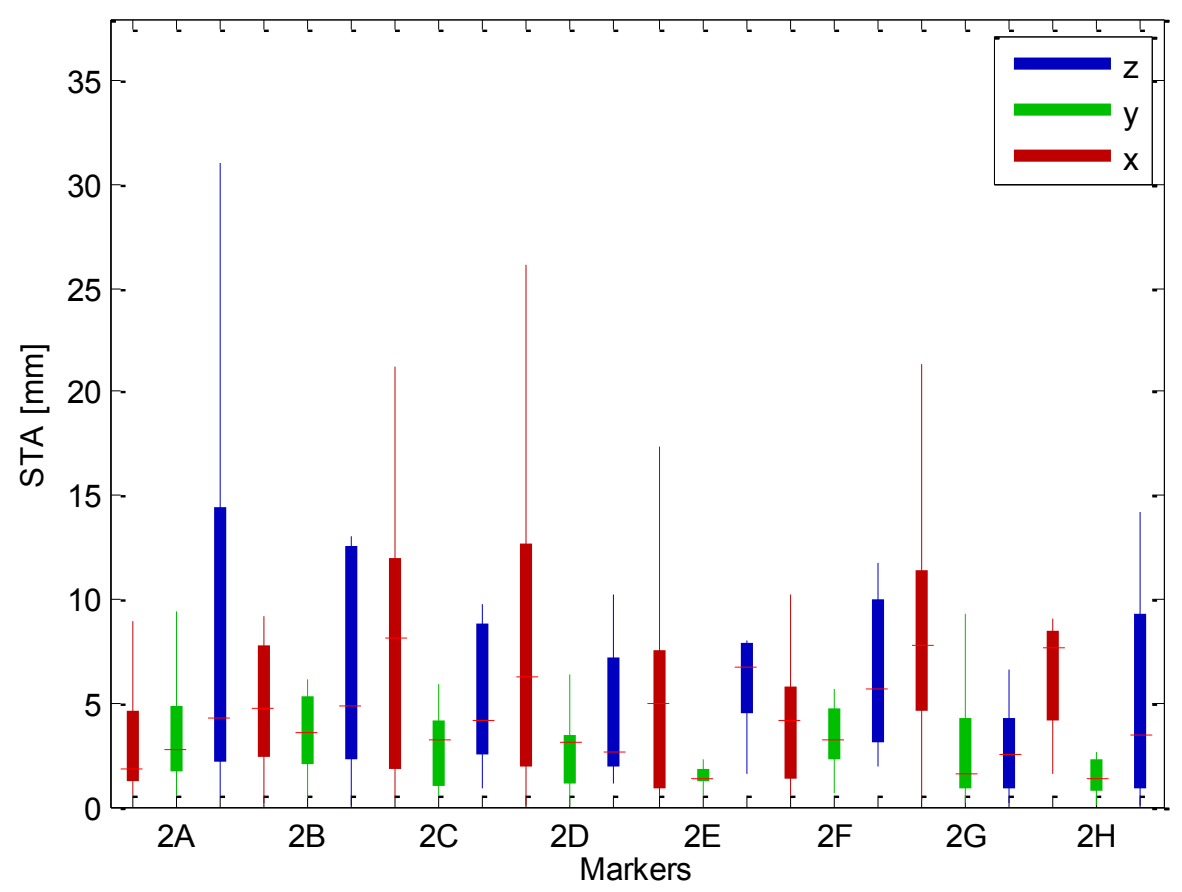

Figure 5.22 - Box-plots of STA Components of the Second Ring of Markers Configuration during Extension 


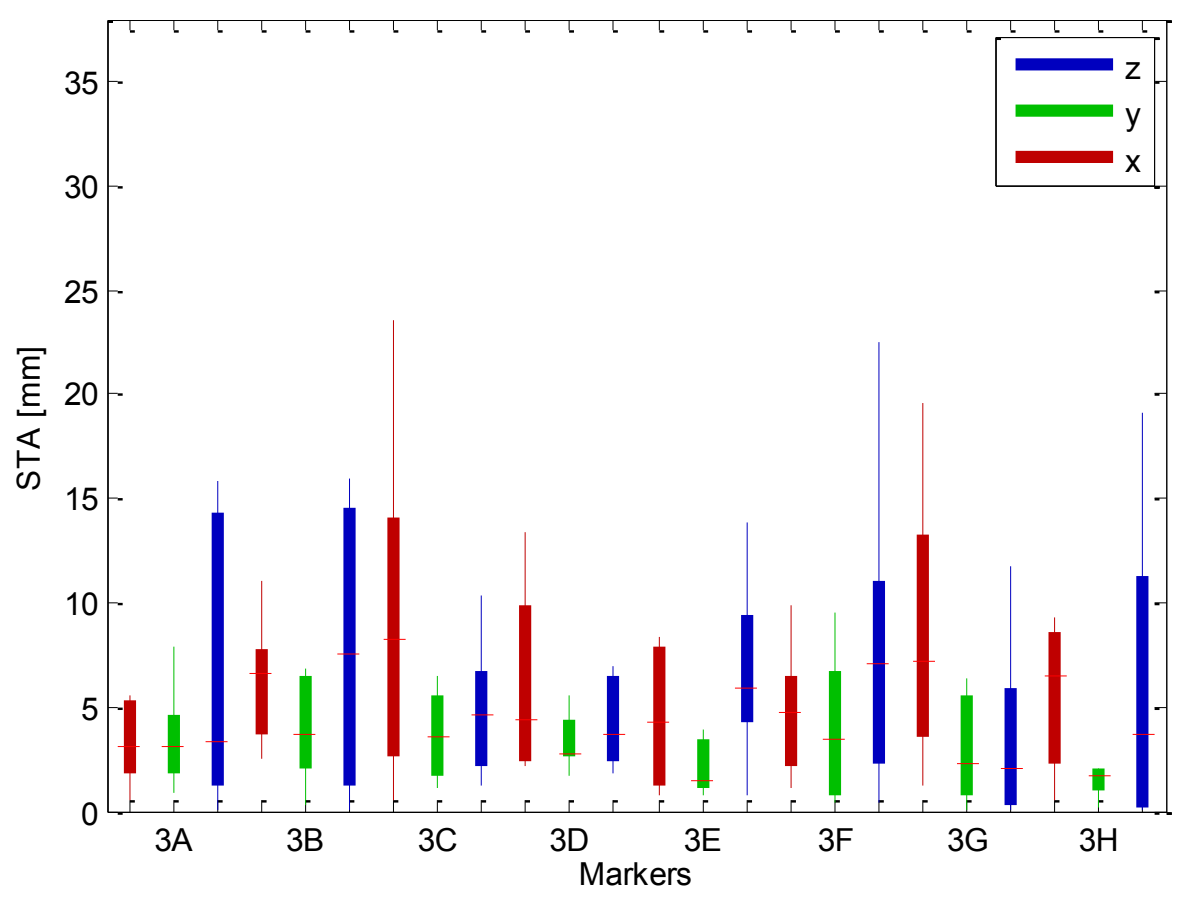

Figure 5.23 - Box-plots of STA Components of the Third Ring of Markers Configuration during Extension

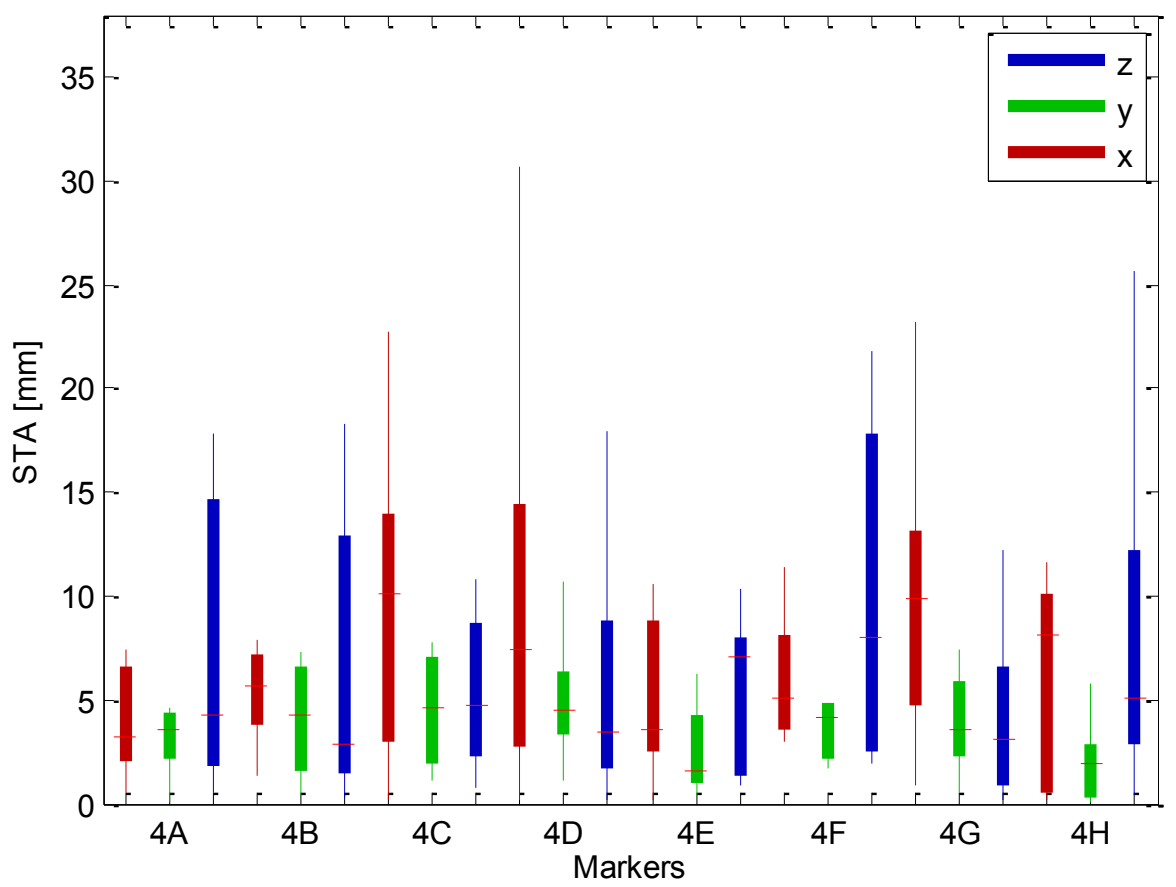

Figure 5.24 - Box-plots of STA Components of the Fourth Ring of Markers Configuration during Extension 


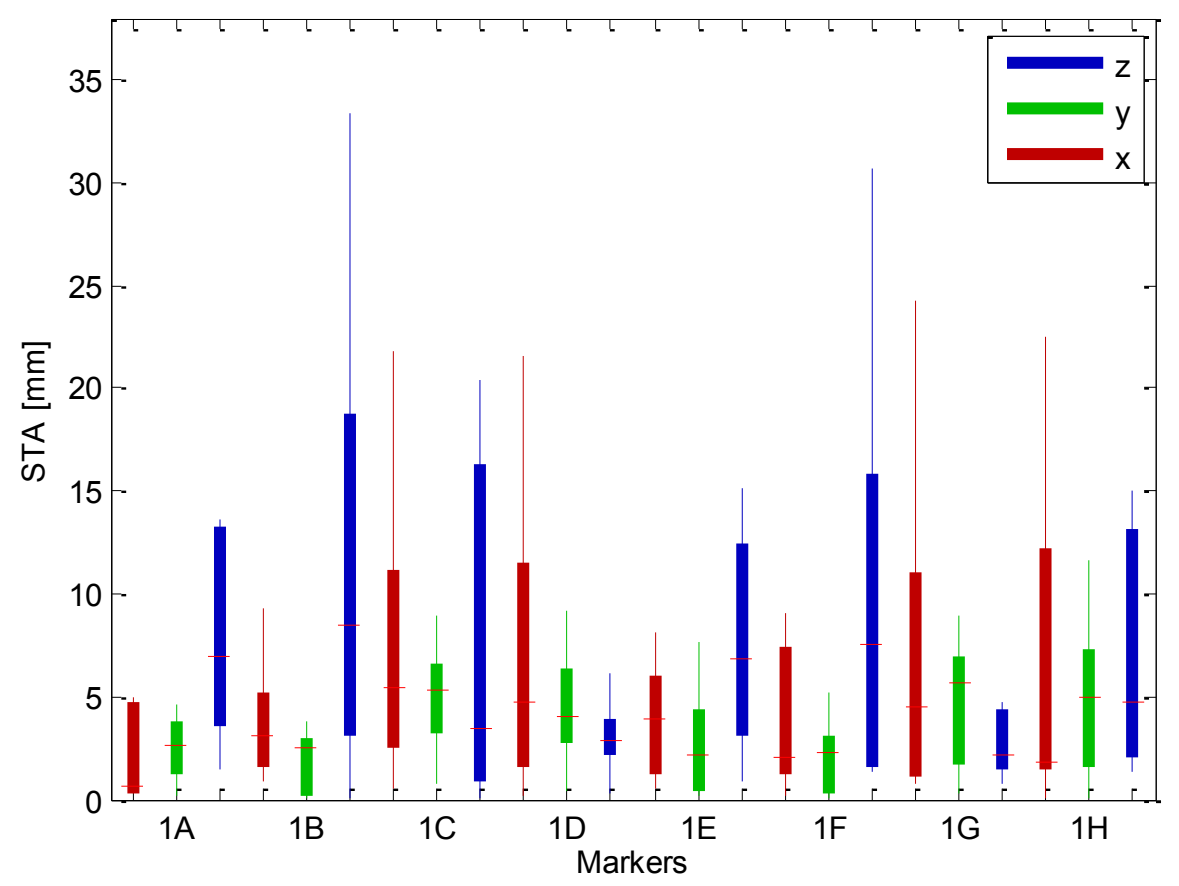

Figure 5.25 - Box-plots of STA Components of the First Ring of Markers Configuration during Abduction

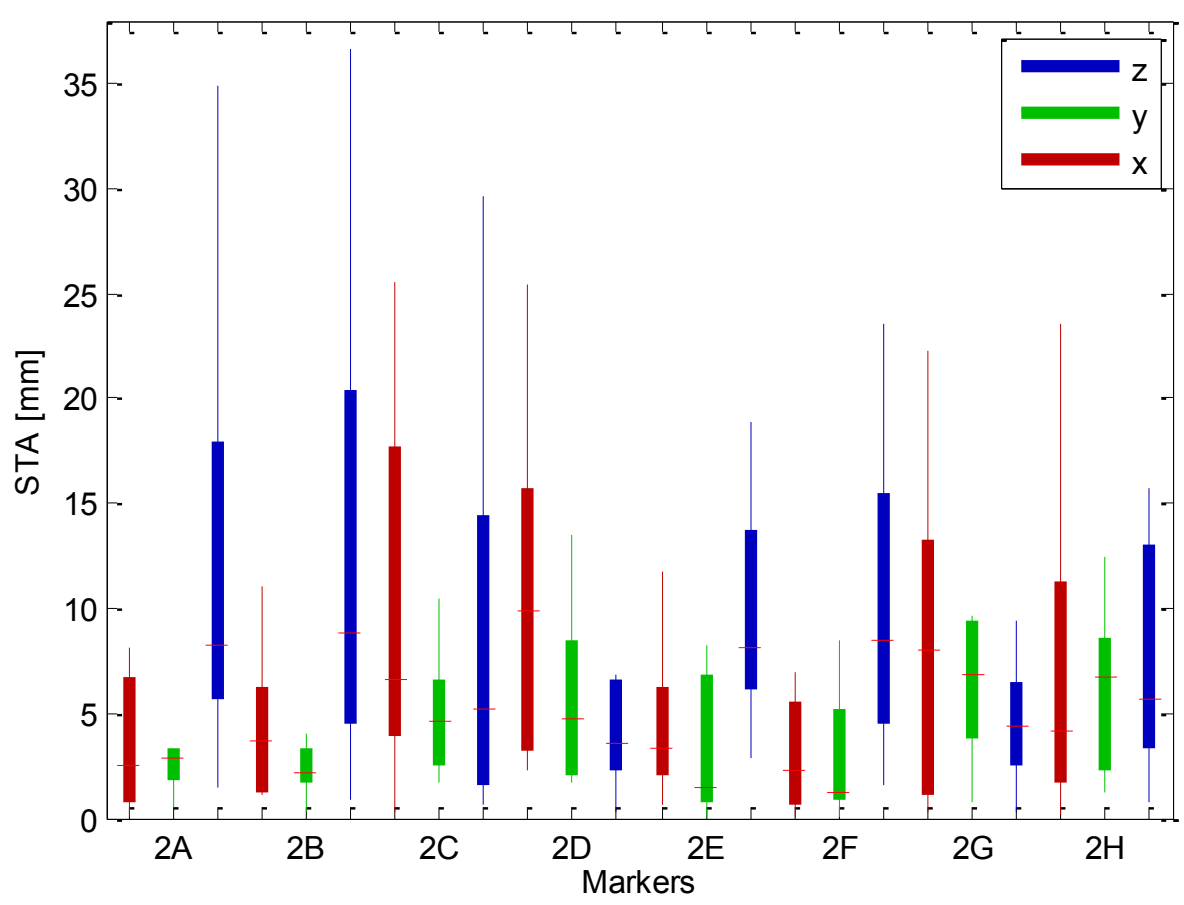

Figure 5.26 - Box-plots of STA Components of the Second Ring of Markers Configuration during Abduction 


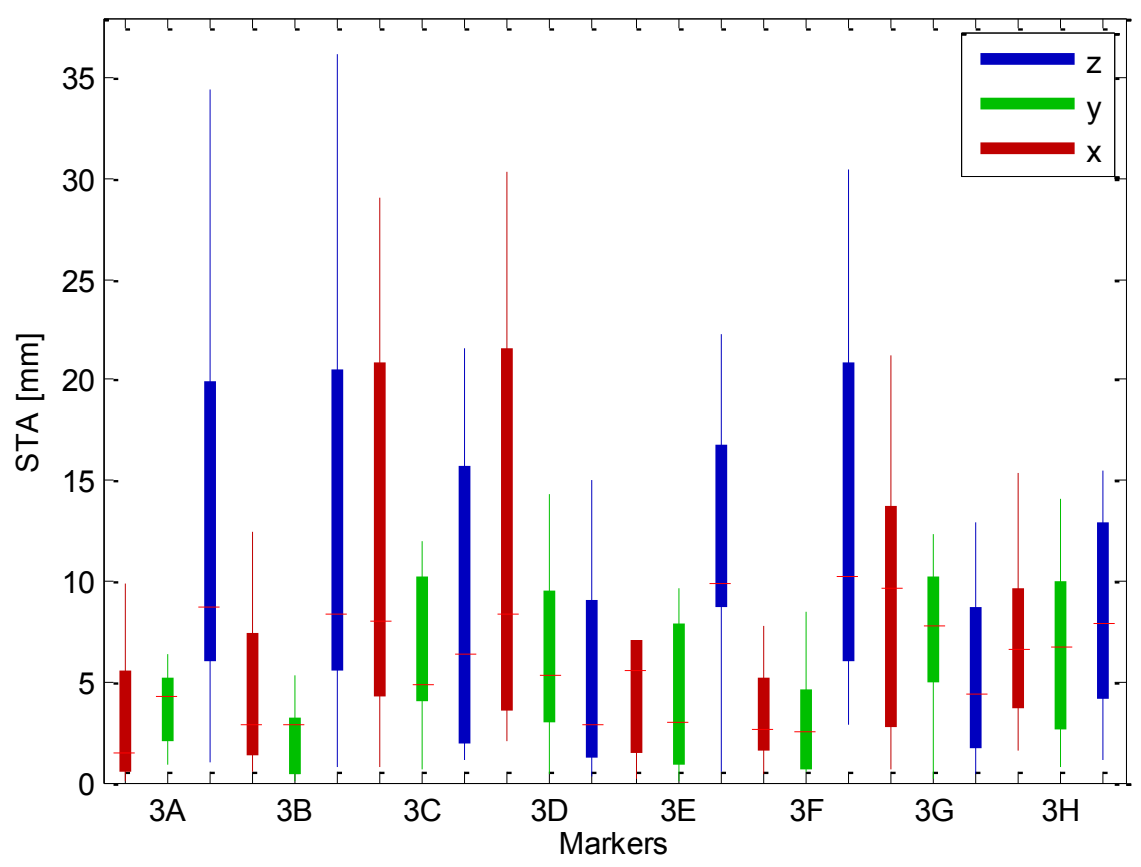

Figure 5.27 - Box-plots of STA Components of the Third Ring of Markers Configuration during Abduction

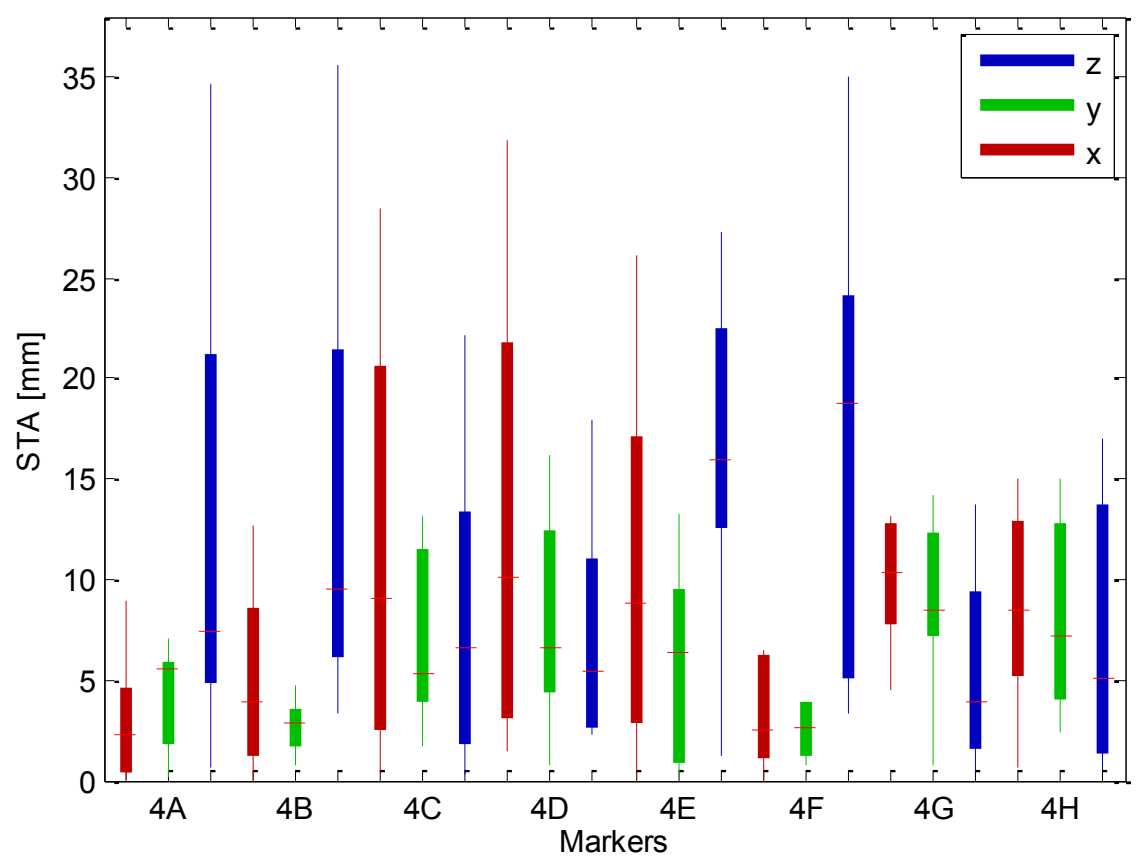

Figure 5.28 - Box-plots of STA Components of the Fourth Ring of Markers Configuration during Abduction 
The assessment of STA was used to correct STA errors to more accurately determination the HJC location using the SCoRE algorithm. Two groups of markers consisting of three non-collinear markers were required to determine HJC using SCoRE algorithm, one group placed on the thigh and the other placed on the pelvis. As discussed before, three key markers have less change in their tissue thickness during all movements; therefore they were considered as the first group of markers attached to the thigh. The second group of markers included the trajectories of markers on the left and right anterior superior iliac spine and the lower spine. The second group of markers were placed on the bony landmarks and they were not affected by STA. In this part, at first we transferred all the markers in a way that the markers on the left and right anterior superior iliac spine and the lower spine match the same markers locations in the other movements. Then we applied the SCoRE algorithm using Equation (27) on the 3 key markers, once on the markers positions before reducing STA and once when we recalculated the markers positions (the positions on the bone) based on the STA quantification. We calculated $\boldsymbol{R}_{1}, \ldots, \boldsymbol{R}_{4}$ and $d_{1}, \ldots, d_{4}$ based on the markers attached to the thigh and $\boldsymbol{S}_{1}, \ldots, \boldsymbol{S}_{4}$ and $t_{1}, \ldots, t_{4}$ based on the markers attached to the pelvis as discussed before. The indices of these parameters indicate four frames which are corresponded to the frames of standing position, flexion, extension and abduction. For each participant during each movement, the SCoRE algorithm returned two centres and the distance between them showed the effectiveness of our method in minimizing STA effects.

Figures 5.29, 5.30, 5.31, and 5.32 show the error in determination of the hip joint centre for all subjects during standing position, flexion, extension and abduction. Each subject has two levels of error; one based on the markers positions before reducing STA and one based on the recalculated positions of the markers after eliminating STA. 


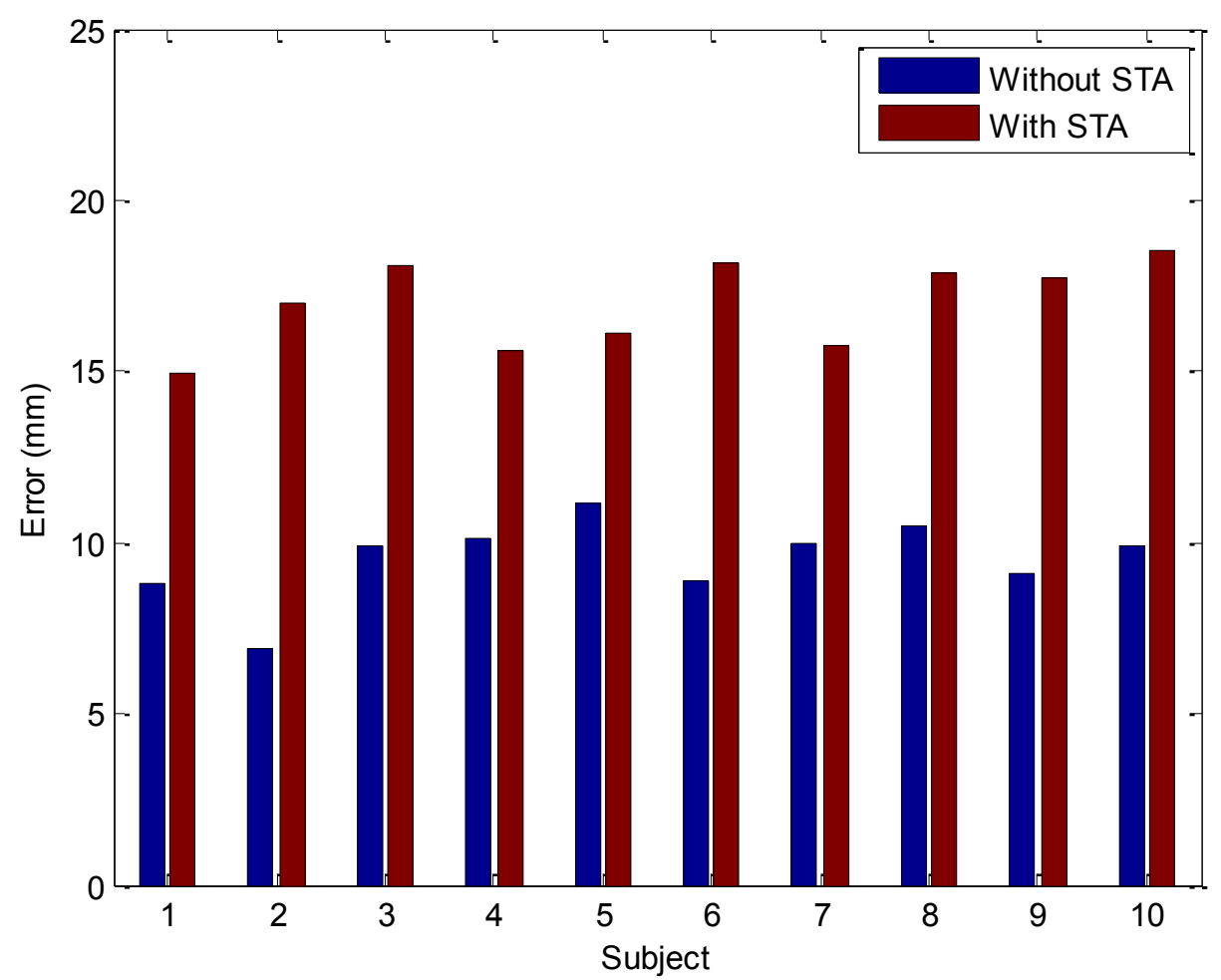

Figure 5.29 - Hip Joint Centre Location Error Using SCoRE Algorithm, Standing Position

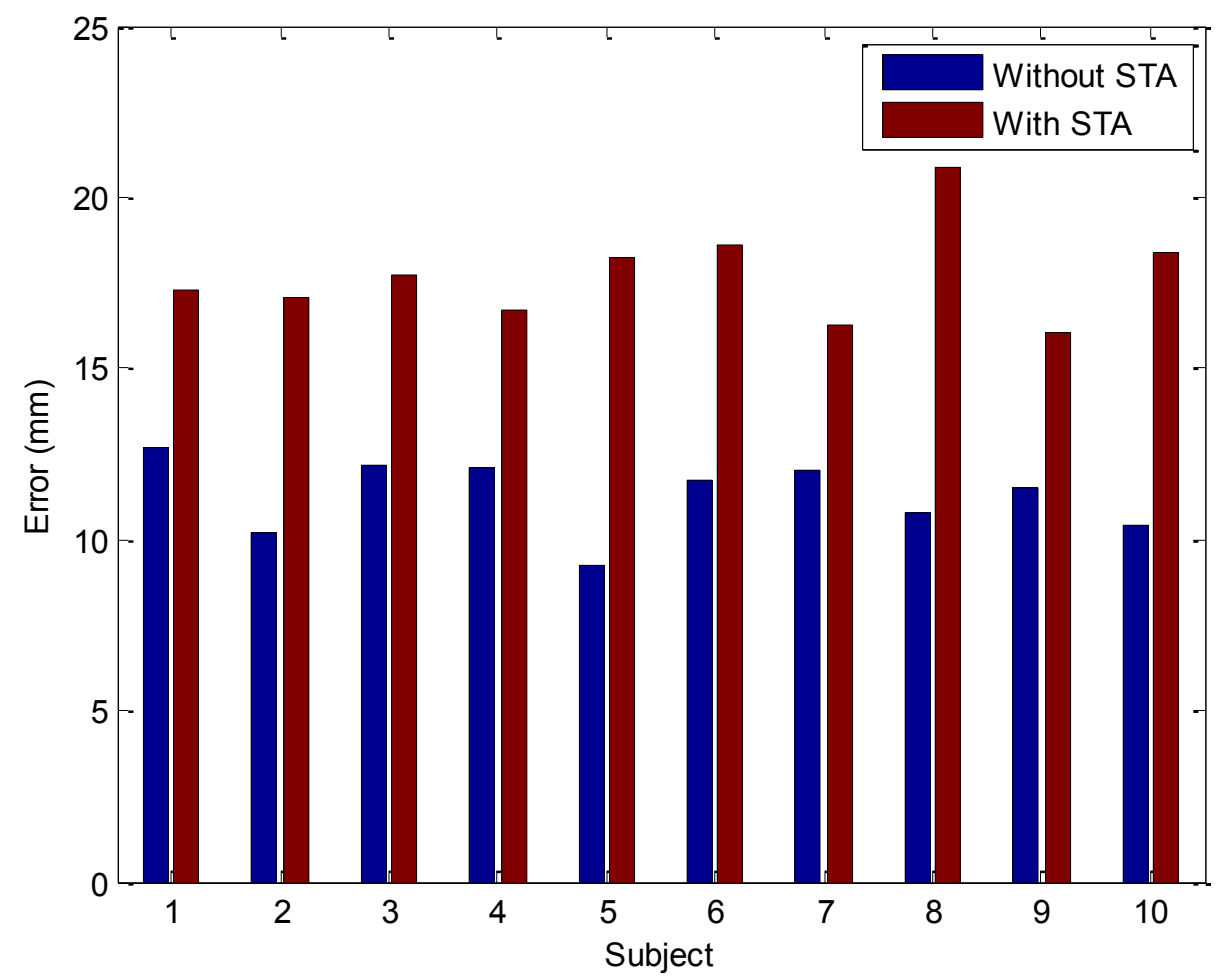

Figure 5.30 - Hip Joint Centre Location Error Using SCoRE Algorithm, Flexion 


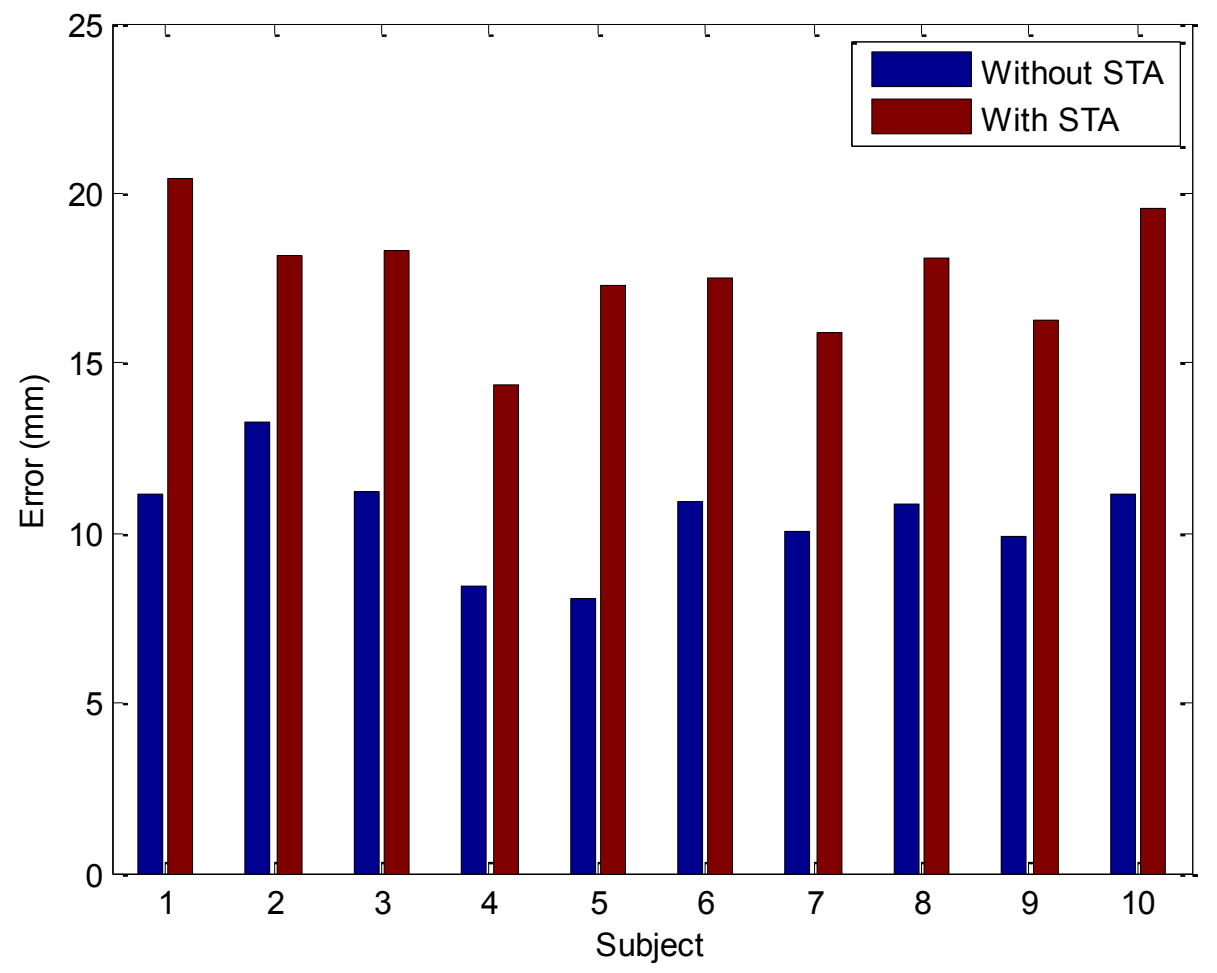

Figure 5.31 - Hip Joint Centre Location Error Using SCoRE Algorithm, Extension

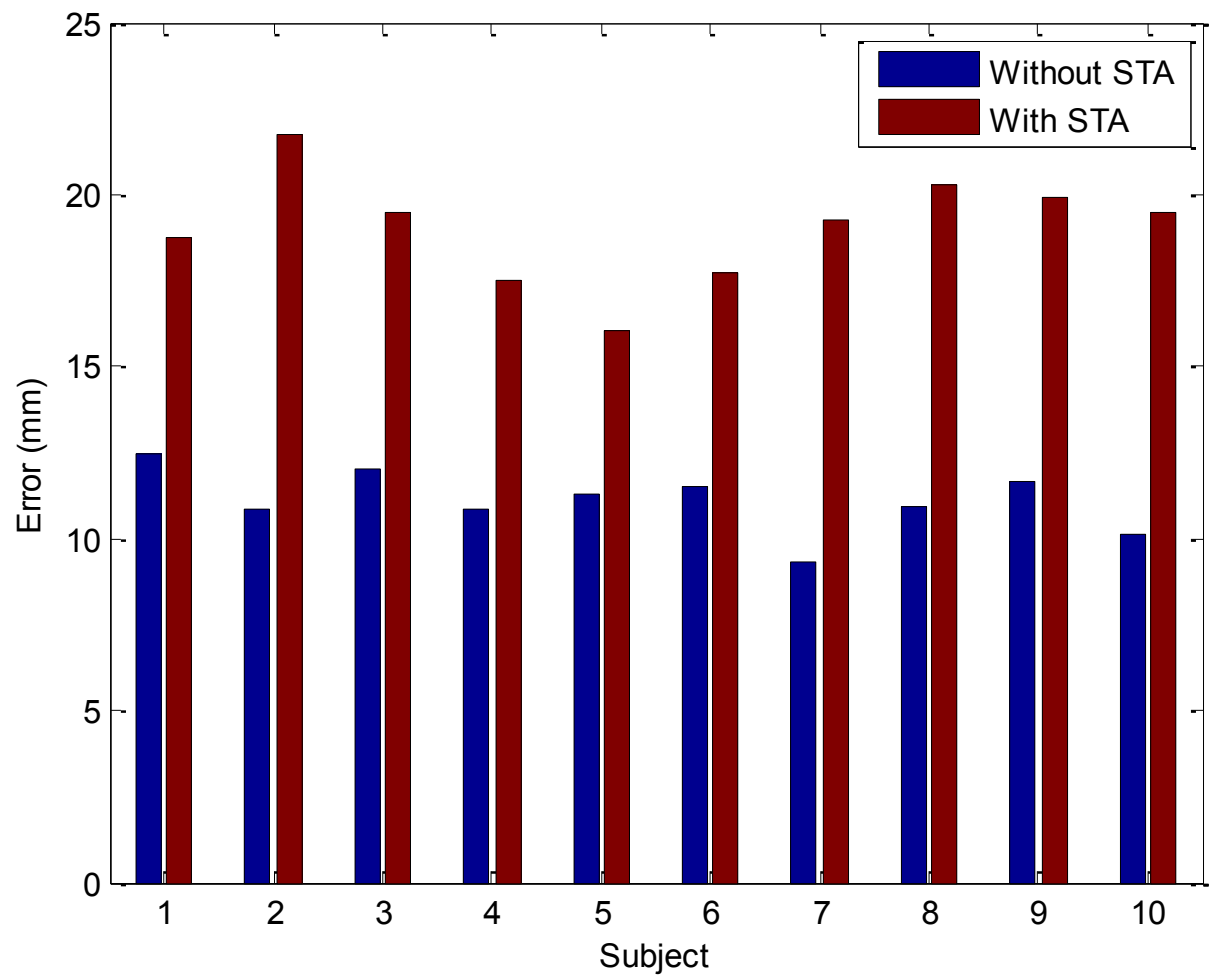

Figure 5.32 - Hip Joint Centre Location Error Using SCoRE Algorithm, Abduction 


\section{Chapter 6: Conclusion}

\subsection{Contributions}

Soft tissue artefact is the most significant source of error in human movement analysis. In this study, we utilized a combined experimental setup of optical motion capture system and ultrasound imaging system. The optical motion capture is the most commonly used system in human movement studies since it tracks trajectories of the markers to have realistic motions of the body non-invasively. Ultrasound is one of the preferred imaging modalities because this modality is non-invasive and poses no harm to human bodies and, is a low cost and portable imaging modality. As optical motion capture system and ultrasound imaging system are non-invasive, our proposed experimental setup is noninvasive and appropriate for clinical daily uses in contrast to many of the previous studies on STA assessment and compensation which were invasive.

Using optical motion capture system along with ultrasound depth measurements data, we quantified STA on ten subjects during three ranges of motions of the hip joint, flexion, extension, and abduction comparing with natural position which was considered standing position. At first, we recorded each marker position placed on the thigh and pelvis for a range of motions of the hip joint. We used ultrasound imaging to measure the changes in tissue thickness at the marker positions for the same standing and extended positions. Three markers were selected as three key markers based on the ultrasound depth measurements. Then we proposed using a piecewise polynomial cubic spline interpolation to fit curves to the markers' positions and applying UDM information in 
order to determine bone positions at the positions of three key markers. We used these positions on the bone to assess STA during several movements of the hip joint.

The results showed the markers displacements were non-linear, subject and task dependent and generally larger in areas closer to the hip joint. The hip is surrounded by several muscles linked to bones via tendons. These muscles provide the joint stability and control body movements. Since different muscles of the hip and thigh produce different movements of the hip, the markers displacements are dependent on the movement. Most of the subjects had relatively larger STA in abduction movement; because different subjects had muscles with different levels of strength.

This STA assessment was used to correct STA errors to more accurately determination of the HJC location using the SCoRE algorithm. For each subject during each movement, two centres of rotation were obtained; one based on markers trajectories before minimizing the STA and one centre after minimizing the STA and recalculating markers trajectories. The error associated with the data before minimizing the STA and after minimizing the STA effects was approximately in the range of $13-23 \mathrm{~mm}$ and $7-14 \mathrm{~mm}$, respectively. The results showed the improvements obtained in our proposed method as it has been reported 15-26 $\mathrm{mm}$ in the previous studies [93, 20].

\subsection{Future Work}

In this study, we quantified STA at several positions around the thigh of ten subjects during flexion, extension, and abduction. In the future studies, it is suggested to derive a function between the STA, the angles of the femur bone relative to the pelvis during all the movements, and the circumference of the subjects thigh based on our results.

We quantified and minimized STA effects in the hip joint kinematics which has the advantage of being applicable to other joints. 


\section{Appendix A Ethics Protocol Clearance}

Carleton University Research Office

Research Ethics Board

1325 Dunton Tower

1125 Colonel By Drive

Ottawa, ON K1S 5B6 Canada

Tel: 613-520-2517

ethics@carleton.ca

\section{Ethics Clearance Form - New Clearance}

This is to certify that the Carleton University Research Ethics Board has examined the application for ethical clearance. The REB found the research project to meet appropriate ethical standards as outlined in the Tri-Council Policy Statement: Ethical Conduct for Research Involving Human, 2nd edition, and the Carleton University Policies and Procedures for the Ethical Conduct of Research.

Date of Clearance: April 30, 2014

Researcher: Azadeh Rouhandeh (Student Research: Master's Student)

Department: Faculty of Engineering and Design\Systems and Computer Engineering University: Carleton University

Research Supervisor (if applicable): Prof. Christopher Joslin

Project Number: 101235

Alternate File Number (if applicable):

Project Title: Correcting for Soft tissue artefact non-invasively and developing a correction method for minimizing soft tissue artefact to estimate hip joint centre location accurately 
Clearance Expires: May 31, 2015

\section{All researchers are governed by the following conditions:}

Annual Status Report: You are required to submit an Annual Status Report to either renew clearance or close the file. Failure to submit the Annual Status Report will result in the immediate suspension of the project. Funded projects will have accounts suspended until the report is submitted and approved.

Changes to the project: Any changes to the project must be submitted to the Carleton University Research Ethics Board for approval. All changes must be approved prior to the continuance of the research.

Adverse events: Should a participant suffer adversely from their participation in the project you are required to report the matter to the Carleton University Research Ethics Board. You must submit a written record of the event and indicate what steps you have taken to resolve the situation.

Suspension or termination of clearance: Failure to conduct the research in accordance with the principles of the Tri-Council Policy Statement: Ethical Conduct for Research Involving Humans, 2nd edition and the Carleton University Policies and Procedures for the Ethical Conduct of Research may result in the suspension or termination of the research project.

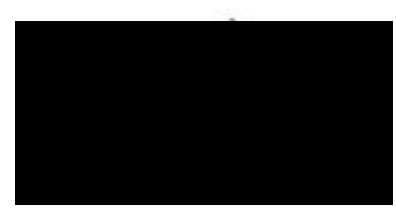

Andy Adler

Chair, Carleton University Research Ethics Board

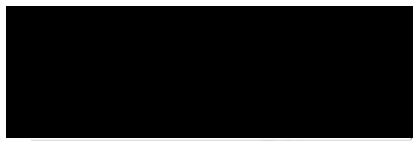

Louise Heslop

Vice-Chair, Carleton University Research Ethics Board 


\section{Appendix B Research Ethics Protocol Application}

雪

Carleton

U N I V E R S I T Y

Canada's Capital University
Research Ethics Board 1325 Dunton Tower Tel: 613-520-2517 ethics@carleton.ca

\section{Office use only:}

Project number:

Type of review: $\square$ Expedited

Date received:

REB Review Date:

\section{Section 1: Project Registration}

\subsection{Project Title:}

Correcting for Soft-Tissue Artefacts Using Prior Ultrasound Measurements and Dynamic Adaptation to Motion Capture Data

\subsection{Purpose of the research (one or two sentences):}

Assessing soft tissue artefact non-invasively and developing a correction method for minimizing soft tissue artefact to estimate hip joint centre location accurately.

1.3 Researcher Information: (Students must also complete the supervisor section)

Name: Azadeh Rouhandeh

Department: Systems and Computer Engineering, Carleton University

University E-mail Address: azadehrouhandeh@cmail.carleton.ca 


\section{Position:}

$\square$ Faculty Member $\quad$ Instructor or Sessional $\quad$ Library staff $\square$ Post-Doctoral Fellow

$\square$ Ph.D. student $\quad$ Master's student $\quad \square 4^{\text {th }}$ year project $\quad \square$ Undergraduate

\subsection{Thesis or Project supervisor (Students only)}

Name: Dr. Chris Joslin

Department: Systems and Computer Engineering, Carleton University

University E-mail Address: Chris_Joslin@carleton.ca

\subsection{Was this project granted an Approval in Principle for the purpose of releasing research funds? $\square$ Yes No}

If Yes: Please provide the project number:

1.6 Is this protocol a main or umbrella application for others projects? $\square$ Yes - No

\subsection{Proposed Research Dates:}

Date to begin recruiting participants: $1 / 4 / 2014$

Date project will conclude: 30/4/2014

1.8 Other researchers: $\square$ Not applicable

\begin{tabular}{|l|l|l|}
\hline Name & E-mail Address & Role \\
\hline & & \\
\hline & & \\
\hline
\end{tabular}

1.8 Student Research Assistants: $\square$ Not applicable $\quad \square$ To be named later

\begin{tabular}{|l|l|l|}
\hline Name & Department/School & E-mail \\
\hline & & \\
\hline & & \\
\hline
\end{tabular}


1.9 Research funding: Is this project funded? $\quad$ Received $\square$ Pending $\square$ Not funded

Funding source and program

NSERC / Collaborative Health Research Project

\section{If funded, will other protocols be submitted under this funding? $\square$ Yes}

- No

\subsection{Researcher signature:}

I agree to conduct this research in accordance with the Tri-Council Policy Statement: Ethical Conduct for Research Involving Humans $2^{\text {nd }}$ edition the Carleton University Policies and Procedures for the Ethical Conduct of Research and the conditions of clearance established by the Carleton University Research Ethics Board.

Signature of researcher

Date

\section{Research supervisor (Student projects only)}

I have assisted with, read and approved the research ethics protocol. I will ensure that the student researcher conducts the research in accordance with the Tri-Council Policy Statement: Ethical Conduct for Research Involving Humans $2^{\text {nd }}$ edition, the Carleton University Policies and Procedures for the Ethical Conduct of Research and the conditions of clearance established by the Carleton University Research Ethics Board and agree to provide all necessary supervision to the student.

Signatur

Date:

\section{Section 2: Research Project Information(Complete all sections below)}

\subsection{Description of research project and its objectives: (Limit 200 words.)}

The centre of rotation of the hip joint is needed for an accurate simulation of the joint performance in many applications such as pre-operative planning simulation, human gait analysis, and hip joint disorders. The hip joint centre location can be determined using two general approaches: predictive methods and functional methods. Predictive methods estimate the hip joint centre based on regression 
equations between palpable bony landmarks and the joint centre. Functional methods are based on the relative motion of the femur to pelvis measured using reflective markers. Functional methods are more accurate and perform significantly better than predictive methods on human subjects. However, the main limitation of functional methods is the relative motion between the markers and bone. This movement is represented as a Soft Tissue Artefact (STA), which is the most critical source of error in the estimation of the hip joint centre. There are various studies associated with describing and compensating soft tissue artefact invasively, such as intra-cortical pins, external fixators, and percutaneous trackers. The goal of this study is to assess the soft tissue artefact non-invasively and develop a correction method for minimizing soft tissue artefact to estimate hip joint centre accurately. To this end, we performed key experiments consisting of ultrasound measurements and motion capture analysis. In our method, the shift attributed to the skin tissue is determined and related algorithmically to the global motion of the leg. This shift is eliminated by using tissue thickness from ultrasound measurements to have an accurate location of the hip joint centre.

2.2 Methodology/Procedures: Describe all methods and procedures that will be used to obtain data

Approximately 10 people will be invited to participate in this study. Participants will be informed of the details of the project both verbally and through a letter of information. They will have the opportunity to ask questions and will have to sign a consent form in accordance to the institutional research ethics and scientific committees. Participants' age, weight, height and body mass index will be recorded as part of the data collection. The study consists of two data collection parts, motion capture and ultrasound measurements. Participants will be asked to wear some appropriate clothes and remove some of their clothes during the tests of this study. The first component of the study is motion capture; reflective markers will be attached to the skin surface of one leg. They will be asked to perform three clinical movements for one leg, Flexion/Extension in sagittal plane, Abduction/Adduction in frontal plane and Internal/External rotation in transverse. Kinematics will be recorded at by 10 vicon optical motion cameras.

The second component is ultrasound measurements to track the changes in the tissue thickness while the participants have the same positions as the first part. This part will be performed by ultrasound imaging machine (Picus, Esaote Europe) and 
linear probe (L10-5, $5 \mathrm{MHz}$ operating frequency).

2.3Location where the research will take place:

- Carleton University $\quad \square$ Region of Ottawa-Carleton

$\square$ Canada (please specify) $\square$ Other (please specify):

\subsection{Will additional ethics reviews be required? $\quad$ No}

If yes, please provide the name, address and contact information/person for each board or committee.

\subsection{Visa or research license required:}

$\square$ Yes. Documentation attached $\quad \square$ Yes. Documentation to follow $\quad$ No

\section{Faculty projects only:}

\subsection{Training and supervision of student researchers: $\quad \square$ Yes $\quad \square$ No}

a) Training/experience: Please outline the training that student researchers will receive before commencing work on this project. Include information about their experience to date and their qualifications to work on the project.

b) Supervision: Please outline the supervision plan for student researchers.

\section{Section 3: Research Participants}

\subsection{Recruitment}

a) Describe how potential research participants will be identified and recruited. (Include information on recruitment methods; i.e. email, letter, poster, etc., and where applicable the location of recruitment.)

Participants will be recruited from people with no functional problems of the lower limb by sending email through student boards and pasting posters around the campus. 


\section{b) Proposed number of participants in the study: Ten}

\section{c) Vulnerable research population: Not applicable}

Describe steps to be taken to ensure that the needs of research participants are respected and the collection of data method will ensure the safe and ethical conduct of the research. Where applicable describe your knowledge, training and experience working with the identified population.

3.2 Append the required recruitment documents. (Letters, Telephone scripts, emails, posters, etc. Please identify each clearly, example: Appendix 1: Letter of Invitation)

\section{Section 4: Conflict of Interest/Power Relationships}

\subsection{Are potential research participants employees, clients or persons you have worked with in a professional or volunteer capacity (past or present)? $\square$ Yes $\quad$ No}

If Yes: Please explain your relationship to or authority over the potential participants and what steps you will take to ensure that the participants' decision to take part in the research will not be influenced by their relationship to you.

\subsection{Are potential research participants, friends, relatives or students? $\square$ Yes $\square$ No}

If Yes: Please explain your relationship to or authority over the potential participants and what steps you will take to ensure that the participants' decision to take part in the research will not be influenced by their relationship to you.

Participants might be friends or fellow students. An information session will be held with them to explain the research and all the steps of the study. They will be told during the session and on the consent form that they can withdraw at any time and their data will be deleted. 


\subsection{Do you or any members of the research team have any financial and/or commercialization interest in the research results? $\square$ Yes

If Yes: Please explain in detail your interest.

\section{Section 5: Risks and Benefits}

5.1 Risks to participants: (Check all that apply)

- Physical harm or discomfort (This includes any bodily contact, application of equipment, management of any substance.)

- Psychological/emotional harm (This includes feeling demeaned, embarrassed worried or upset.)

Social and economic harm (This includes loss of status, reputation, ridicule form peers, family or friends.)

Economic risk (This includes loss of income, threat to employment, loss of work time)

$\square$ Legal risk (This includes possible criminal charges or legal suit, duty to report.)

- Other inconveniences (This includes travel costs, disruption to normal activities, time commitment, etc.)

\section{Explain in detail all possible risks to research participants.}

The study has no risk to participants. In the first step of the study, surface capturing of one leg will be taken. In the second part, reflective markers will be placed on one leg of participants and they will be asked to perform some movements. All these movements will be performed without pain at subject's own pace and ability. In the third part, tissue thickness will be measured by ultrasound imaging which is a noninvasive modality. Consequently, all parts of this study have no harms and risks to participants. 
5.2 Managing risk: Describe what steps will be taken to reduce harm to participants.

Participants will have the option to withdraw from the study if they cannot perform the movements without pain. They will be provided private changing area and their friends can help/accompany them.

5.3 Deception: Is there any deception involved in this research project? $\square$ Yes - No

If Yes: Please describe why participants will be deceived, how the deception will be carried out and how and when you will debrief participants.

5.4 Are there any risks to you or the research team? $\square$ Yes

If Yes: Please describe the steps that will be taken to ensure researcher safety.

\subsection{Are there any benefits to the research participants? $\square$ Yes $\square$ No}

If Yes: Please describe those benefits.

Participants can be informed and aware of the possible functional problems of the lower limb.

5.6 If you are working with an agency or community group describe what benefits they may receive from this research.

\section{Section 6: Compensation/Remuneration}

\subsection{Will research participants (including an organization) receive compensation or remuneration for their participation? $\quad \square$ Yes No} If Yes:

a) Describe the compensation/remuneration (money, gift, transportation, childcare costs, etc.) 
b) What is the monetary value of the compensation/remuneration?

6.2 Will you or members of the research team provide time or assistance to the community participants? $\square$ Yes No

If Yes: Please describe the assistance that will be provided.

\section{Section 7: Anonymity and Confidentiality}

7.1 Anonymity (Treatment of the identity of participants)

a) Will the identity of participants be known to the researcher(s) during the collection of personal information, data gathering or testing?
- Yes
$\square$ No

b) Will the identity of participants be revealed in any reports, papers, research articles, recordings, photographs or presentations, etc?

$\square$ Yes $\quad$ No

c) Will the identity of participants be known to other participants in the study?
$\square$ Yes
- No

d) Will the identity of participants be known to non-participants in the study? (colleagues, family friends of the participants)

$\square$ Yes

No

7.2 Confidentiality: (Attribution of responses and data)

a) The responses/data collected will not be attributed to participants.
- Yes
$\square$ No

b) The data collected will be attributed to participants.

$\square$ Yes

- No

If YES to $b$ ):

Will participants have the opportunity to request that certain responses remain nonattributable?
$\square$ Yes
$\square$ No 
7.3 Limitations on anonymity and confidentiality: If the researcher(s) anticipate a situation that may result in a duty to report the names of participants and other relevant information please describe those circumstances here.

\section{Section 8: Informed Consent}

8.1 Describe the procedures for obtaining informed consent for each part of the research project.

The researchers will conduct an information session with volunteers to explain the research, all the steps of the study and answer any questions. After that if they want to participate this research, they will be asked to sign the written informed consent.

8.2 Participant withdrawal: (Please explain the procedures for participants to withdraw from the research study.)

Participants will be told during the informational session and on the consent form that they can withdraw at any time. Any data or information from participants that have withdrawn will be destroyed unless otherwise permitted by the participants.

\subsection{Future use of data:}

8.4 Append the required informed consent form (For Carleton researchers all consent forms must be on Carleton University letterhead.)

Section 9: Data Collection, Storage and Dissemination

9.1. On-line survey section only: not applicable

Is the host survey company Canadian? $\square$ Yes $\quad \square$ No 
If No, in what country will the host data be stored?

Describe the process for transferring the data from the host server to you and verification that the host server is no longer in possession of the data.

\subsection{Audio, video recording or photographs}

Will the research participants be audio, video recorded or photographed? $\mathbf{Y}$ Yes

$\square$ No

If Yes: Please describe how the recordings or photographs will be used. If they are to be used in any presentations of the research participants must be made aware of this and consent to their use. If recordings or photographs are to be destroyed explain how and when this will occur.

Digital pictures may be taken of one or more participants in order to demonstrate the tasks for all parts of the study. Pictures will only be taken if participants consent to it. Faces will be concealed so that participants cannot be identified.

\subsection{Translation and transcription of data:}

Will the research project require the services of a translator or transcriber? (Check No if researcher is translator) $\quad \square$ Yes $\quad$ No

If Yes: please describe what steps will be taken to ensure the privacy and confidentiality of the participants. Please provide a copy of the confidentiality agreement for the translator or transcriber.

9.4 Storage of data: (Explain how the data will be stored during the course of the study. If the data will be used in future studies explain how it will be stored.)

The data will be stored file-based for each part of the study and each participant.

9.5 Access to data: (Explain who will have access to the data during the course of the study.) 
Only the researchers will have access to the data during the course of the study.

9.6 Disposition of Data (After analysis and completion of report)

Data will be:

Returned to participants (Describe in what format the data will be returned and approximate date it will be returned to participants.)

Destroyed (Describe how the data will be destroyed and approximate date of destruction. This includes all audio tapes, digital recordings, videos and photographs.)

Data to be stored and used in future projects (Describe how the data will be stored; including the storage format and how it may be used in future research projects. NOTE: the data can only be used in the same or related projects.)

The data will be stored on an encrypted data drive, with anonymous participant information, and will be used for further analysis of the centre of rotation.

9.7Dissemination: (Check all that apply)

Academic journals $\quad \square$ Web site/publication (open to the public)

$\operatorname{Book}(\mathrm{s})$

- Workshops

- Conferences

$\square$ Classroom presentations/exercises

口Final report to organization

- Final report to participants, upon request

\section{Section 10: Research Instrument}

Append a copy of all research instruments for the project. This includes questionnaires, interview guides, sample questions, written tests and assignments, descriptions of apparatus and equipment. 


\title{
Appendix C Consent Form
}

\author{
Carleton \\ U N I V E R S I T Y \\ Canada's Capital University
}

Title: Correcting for Soft-Tissue Artefacts Using Prior Ultrasound Measurements and Dynamic Adaptation to Motion Capture Data

Date of ethics clearance: April 30, 2014

Ethics Clearance for the Collection of Data Expires: May 31, 2015

I choose to participate in a study on "Correcting for Soft-Tissue Artefacts Using Prior Ultrasound Measurements and Dynamic Adaptation to Motion Capture Data". This study aims to assess soft tissue artefact non-invasively and develop a correction method for minimizing soft tissue artefact to estimate hip joint centre location accurately. The researcher for this study is Azadeh Rouhandeh in the Systems and Computer Engineering department.

She is working under the supervision of Dr. Chris Joslin in the Systems and Computer Engineering Department.

The purpose of this study is to assess soft tissue artefact non-invasively and develop a correction method for minimizing soft tissue artefact to estimate hip joint centre location accurately. To this end, the study consists of three data collection components: surface capture, motion capture, and ultrasound measurements. You will be asked to wear some appropriate clothes and remove some of your clothes during the tests of this study.

The study has no risk to you and involves one hour experiment. In the first 
component, tissue thickness will be measured by ultrasound imaging machine which is a non-invasive modality. In the second component, reflective markers will be placed on the same leg and you will be asked to perform some movements. All these movements will be performed without pain and at your own pace and ability (no extended range is required). Consequently, all parts of this study pose no harm and risk to you.

The location for data collection is Carleton University.

Your identity will be known to the researcher and the research supervisor during the collection of information, data gathering, and testing but will not be revealed in any reports, papers, research articles, presentations or any other participants in the study.

You can withdraw at any time and the data or information will be destroyed unless otherwise permitted by yourself.

All data will be stored file-based, encrypted, and password-protected. The data will only be accessible by the researcher and the research supervisor.

Once the project is completed, all research data will be stored anonymously for a period of 10 Years, after which time all data will be destroyed through aunreversible process appropriate at the time of destruction.

This project was reviewed by the Carleton University Research Ethics Board, which provided clearance to carry out the research. Should you have questions or concerns related to your involvement in this research, please contact:

\section{REB contact information:}

Professor Andy Adler, Chair

Professor Louise Heslop, Vice-Chair

Research Ethics Board

Carleton University

1325 Dunton Tower

1125 Colonel By Drive

Ottawa, ON K1S 5B6

Tel: 613-520-2517

ethics@carleton.ca 


\section{Researcher contact information:}

Name Azadeh Rouhandeh

Department SCE

Carleton University

Tel: $6135202600 \times 8910$

Email: azadehrouhandeh@cmail.carleton.ca

Do you agree to be audio-recorded:

Yes No
Signature of participant

Signature of researcher

\section{Supervisor contact information:}

Name Dr. Chris Joslin

Department SCE

Carleton University

Tel: $6135202600 \times 1889$

Email: chris joslin@carleton.ca

Date

Date 


\section{References}

[1] R. Ganz, J. Parvizi, M. Beck, M. Leunig, H. Nötzli and K. Siebenrock, "Femoroacetabular Impingement: A Cause for Osteoarthritis of the Hip," Clinical Orthopaedics \& Related Research, vol. 417, pp. 112-120, 2003.

[2] C. W. Pfirrmann, B. Mengiardi, C. Dora, F. Kalberer, M. Zanetti and J. Hodler, "Cam and Pincer Femoroacetabular Impingement: Characteristic MR Arthrographic Findings in 50 Patients," Radiology-Radiological Society of North America, vol. 240, no. 3, pp. 778-785, 2006.

[3] H. P. L. Bäthis, M. Tingart, C. Lüring, D. Zurakowski and J. Grifka, "Alignment in Total Knee Arthroplasty A comparison of Computer-assisted Surgery with the Conventional Technique," Journal of Bone \& Joint Surgery, British Volume, vol. 86, no. 5, pp. 682-687, 2004.

[4] E. M. Arnold, S. R. Ward, R. L. Lieber and S. L. Delp, "A Model of the Lower Limb for Analysis of Human Movement," Annals of biomedical engineering, vol. 38, no. 2, pp. 269-279, 2010.

[5] R. N. Kirkwood, E. G. Culham and P. Costigan, "Radiographic and Non-invasive Determination of the Hip Joint Center Location: Effect on Hip Joint Moments," Clinical Biomechanics, vol. 14, no. 4, pp. 227-235, 1999.

[6] A. Leardini, A. Cappozzo, F. Catani, S. Toksvig-Larsen, A. Petitto, V. Sforz, G. Cassanelli and S. Giannini, "Validation of a Functional Method for the Estimation of Hip Joint Centre Location," Journal of Biomechanics, vol. 32, no. 1, pp. 99-103, 1999.

[7] A. D. Speirs, D. L. Benoit, M. L. Beaulieu, M. Lamontagne and P. E. Beaulé, "The 
Accuracy of the Use of Functional Hip Motions on Localization of the Center of the Hip," HSS Journal®, vol. 8, no. 3, pp. 192-197, 2012.

[8] A. L. Bell, R. A. Brand and D. R. Pedersen, "Prediction of Hip Joint Centre Location from External Landmarks," Human Movement Science, vol. 8, no. 1, pp. 3-16, 1989.

[9] V. Camomilla, A. Cereatti, G. Vannozzi and A. Cappozzo, "An optimized Protocol for Hip Joint Centre Determination Using the Functional Method," Journal of Biomechanics, vol. 39, no. 6, pp. 1096-1106, 2006.

[10] V. Bouffard, M. Begon, A. Champagne, P. Farhadnia, P. A. Vendittoli, M. Lavigne and F. Prince, "Hip Joint Center Localisation: A Biomechanical Application to Hip Arthroplasty Population," World Journal of Orthopedics, vol. 3, no. 8, p. 131, 2012.

[11] A. Leardini, L. Chiari, U. D. Croce and A. Cappozzo, "Human Movement Analysis Using Stereophotogrammetry: Part 3. Soft Tissue Artifact Assessment and Compensation," Gait \& Posture, vol. 21, no. 2, pp. 212-225, 2005.

[12] M. Sangeux, F. Marin, F. Charleux, L. Dürselen and M. C. Ho Ba Tho, "Quantification of the 3D Relative Movement of External Marker Sets vs. Bones Based on Magnetic Resonance Imaging," Clinical Biomechanics, vol. 21, no. 9, pp. 984-991, 2006.

[13] L. Yahia-Cherif, B. Gilles, T. Molet and N. Magnenat-Thalmann, "Motion Capture and Visualization of the Hip Joint with Dynamic MRI and Optical Systems," Computer Animation and Virtual Worlds, vol. 15, no. 3-4, pp. 377-385, 2004.

[14] L. Cheze, B. J. Fregly and J. Dimnet, "A Solidification Procedure to Facilitate Kinematic Analyses based on Video System Data," Journal of Biomechanics, vol. 28, no. 7, pp. 879-884, 1995.

[15] A. Cappello, R. Stagni, S. Fantozzi and A. Leardini, "Soft Tissue Artifact Compensation in Knee Kinematics by Double Anatomical Landmark Calibration: Performance of a Novel Method during Selected Motor Tasks," IEEE Transactions on Biomedical Engineering, vol. 52, no. 6, pp. 992-998, 2005.

[16] E. J. Alexander and T. P. Andriacchi, "Correcting for Deformation in Skin-based Marker Systems," Journal of Biomechanics, vol. 34, no. 3, pp. 355-361, 2001. 
[17] A. Cereatti , U. Della Croce and A. Cappozzo, "Reconstruction of Skeletal Movement Using Skin Markers: Comparative Assessment of Bone Pose Estimators," Journal of NeuroEngineering and Rehabilitation [Online], vol. 3, 2006.

[18] T. W. Lu and J. J. O'connor, "Bone Position Estimation from Skin Marker Coordinates Using Global Optimisation with Joint Constraints," Journal of Biomechanics, vol. 32, no. 2, pp. 129-134, 1999.

[19] R. Stagni, S. Fantozzi and A. Cappello, "Double Calibration vs. Global Optimisation: Performance and Effectiveness for Clinical Application," Gait \& Posture, vol. 29, no. 1, pp. 119-122, 2009.

[20] M. Sangeux, P. Alana and R. Baker, "Hip Joint Centre Localization: Evaluation on Normal Subjects in the Context of Gait Analysis," Gait \& Posture, vol. 34, no. 3, pp. 324-328, 2011.

[21] A. Peters, R. Baker and M. Sangeux, "Validation of 3-D Freehand Ultrasound for the Determination of the Hip Joint Centre," Gait \& Posture, vol. 31, no. 4, pp. 530-532, 2010.

[22] S. Upadhyaya, "Decreasing Error in Functional Hip Joint Center Calculation using Ultrasound Imaging," M.S thesis, School of Information Technology and Engineering, University of Ottawa, Ottawa, 2013.

[23] R. M. Ehrig, W. R. Taylor, G. N. Duda and M. O. Heller, "A Survey of Formal Methods for Determining the Centre of Rotation of Ball Joints," Journal of Biomechanics, vol. 39, no. 15, pp. 2798-2809, 2006.

[24] J. K. Aggarwal and Q. Cai, "Human Motion Analysis: A Review," Computer Vision and Image Understanding, vol. 73, no. 3, pp. 428-440, 1999.

[25] N. Rydell, "Biomechanics of the Hip-joint," Clinical Orthopaedics and Related Research, vol. 92, pp. 6-15, 1973.

[26] M. Alexander, Introduction to Human Anatomy, New York: Thieme-Stratton Inc., 1932.

[27] D. P. Byrne, K. J. Mulhall and J. F. Baker, "Anatomy \& Biomechanics of the Hip," The Open Sports Medicine Journal, vol. 4, pp. 51-57, 2010. 
[28] G. D. Zuidema, The Johns Hopkins Atlas of Human Functional Anatomy, The Johns Hopkins University Press, 1997.

[29] B. Landau, Essential Human Anatomy and Physiology, Scott, Foresman and Company, 1976.

[30] M. H. Arokoski, M. Haara, H. J. Helminen and J. P. Arokoski, "Physical Function in Men with and without Hip Osteoarthritis," Archives of Physical Medicine and Rehabilitation, vol. 85, no. 4, pp. 574-581, 2004.

[31] M. Tannast, M. Kubiak-Langer, F. Langlotz, M. Puls, S. B. Murphy and K. A. Siebenrock, "Noninvasive Three-dimensional Assessment of Femoroacetabular Impingement," Journal of Orthopaedic Research, vol. 25, no. 1, pp. 122-131, 2007.

[32] S. M. Bierma-Zeinstra, A. M. Bohnen, R. Ramlal, J. Ridderikhoff, J. Verhaar and A. Prins, "Comparison between Two Devices for Measuring Hip Joint Motions," Clinical Rehabilitation, vol. 12, no. 6, pp. 497-505, 1998.

[33] S. Nussbaumer, M. Leunig, J. F. Glatthorn, S. Stauffacher, H. Gerber and N. A. Maffiuletti, "Validity and Test-retest Reliability of Manual Goniometers for Measuring Passive Hip Range of Motion in Femoroacetabular Impingement Patients," BMC Musculoskeletal Disorders, vol. 11, no. 1, p. 194, 2010.

[34] D. C. Boone and S. P. Azen, "Normal Range of Motion of Joints in Male Subjects," The Journal of Bone \& Joint Surgery, vol. 61, no. 5, pp. 756-759, 1979.

[35] C. Reinschmidt, A. J. Van Den Bogert, A. Lundberg, B. M. Nigg and N. Murphy, "Tibiofemoral and Tibiocalcaneal Motion during Walking: External vs. Skeletal Markers," Gait \& Posture, vol. 6, no. 2, pp. 98-109, 1997.

[36] M. A. Lafortune, P. R. Cavanaugh, H. J. Sommer and A. Kalenak, "Threedimensional Kinematics of the Human Knee during Walking," Journal of Biomechanics, vol. 25, pp. 347-357, 1992.

[37] M. A. Lafortune, P. R. Cavanagh, H. J. Sommer and A. Kalenak, "Foot InversionEversion and Knee Kinematics During Walking," Journal of Orthopaedic Research, vol. 12, pp. 412-420, 1994.

[38] C. Reinschmidt, A. J. Van Den Bogert, B. M. Nigg, A. Lundberg and N. Murphy, 
"Effect of Skin Movement on the Analysis of Skeletal Knee Joint Motion during Running," Journal of Biomechanics, vol. 30, no. 7, pp. 729-732, 1997.

[39] A. Cappozzo, F. Catani, A. Leardini, M. G. Benedetti and U. Della Croce, "Position and Orientation in Space of Bones during Movement: Experimental Artefacts," Clinical Biomechanics, vol. 11, no. 2, pp. 90-100, 1996.

[40] J. P. Holden, J. A. Orsini, K. L. Siegel, T. M. Kepple, L. H. Gerber and S. J. Stanhope, "Surface Movement Errors in Shank Kinematics and Knee Kinetics during Gait," Gait \& Posture, vol. 5, no. 3, pp. 217-227, 1997.

[41] G. Selvic, "Roentgen Stereophotogrammetry: A Method for the Study of the Kinematics of the Skeletal System," Acta Orthopaedica, vol. 60, no. 232, pp. 1-51, 1989.

[42] E. R. Valstar, R. G. Nelissen, J. H. Reiber and P. M. Rozing, "The Use of Roentgen Stereophotogrammetry to Study Micromotion of Orthopaedic Implants," ISPRS Journal of Photogrammetry and Remote Sensing, vol. 56, no. 5, pp. 376-389, 2002.

[43] S. A. Banks and W. A. Hodge, "Accurate Measurement of Three-dimensional Knee Replacement Kinematics Using Single-plane Fluoroscopy," IEEE Transactions on Biomedical Engineering, vol. 43, no. 6, pp. 638-649, 1996.

[44] A. Menache, Understanding Motion Capture for Computer Animation and Video Games, San Diego: Academic Press, 2000.

[45] R. E. Mayagoitia, A. V. Nene and P. H. Veltink, "Accelerometer and Rate Gyroscope Measurement of Kinematics: An Inexpensive Alternative to Optical Motion Analysis Systems," Journal of Biomechanics, vol. 35, no. 4, pp. 537-542, 2002.

[46] S. Corazza, L. Muendermann, A. M. Chaudhari, T. Demattio, C. Cobelli and T. P. Andriacchi, "A Markerless Motion Capture System to Study Musculoskeletal Biomechanics: Visual Hull and Simulated Annealing Approach," Annals of Biomedical Engineering, vol. 34, no. 6, pp. 1019-1029, 2006.

[47] L. Mündermann, S. Corazza and T. P. Andriacchi, "The Evolution of Methods for the Capture of Human Movement Leading to Markerless Motion Capture for 
Biomechanical Applications," Journal of Neuroengineering Rehabilitation, vol. 3, no. 6, 2006.

[48] H. Dejnabadi, B. M. Jolles and K. Aminian, "A New Approach to Accurate Measurement of Uniaxial Joint Angles based on a Combination of Accelerometers and Gyroscopes," IEEE Transactions on Biomedical Engineering, vol. 52, no. 8, pp. 1478-1484, 2005.

[49] A. T. M. Willemsen, J. A. Van Alste and H. B. K. Boom, "Real-time Gait Assessment Utilizing a New Way of Accelerometry," Journal of Biomechanics, vol. 23, no. 8, pp. 859-863, 1990.

[50] A. Heyn, R. E. Mayagoitia, A. V. Nene and P. H. Veltink, "The Kinematics of the Swing Phase Obtained from Accelerometer and Gyroscope Measurements," Proceedings of the 18th Annual International Conference of the IEEE, vol. 2, pp. 463-464, 1996.

[51] L. Y. Chang and N. S. Pollard, "Constrained Least-squares Optimization for Robust Estimation of Center of Rotation," Journal of Biomechanics, vol. 40, no. 6, pp. 1392-1400, 2007.

[52] S. J. Piazza, N. Okita and P. R. Cavanagh, "Accuracy of the Functional Method of Hip Joint Center Location: Effects of Limited Motion and Varied Implementation," Journal of Biomechanics, vol. 34, no. 7, p. 967-973, 2001.

[53] G. Wu, S. Siegler, P. Allard, C. Kirtley, A. Leardini, D. Rosenbaum, M. Whittle, D. D'Lima, L. Cristofolini, H. Witte, O. Schmid and I. Strokes, "ISB Recommendation on Definitions of Joint Coordinate System of Various Joints for the Reporting of Human Joint Motion-Part I: Ankle, Hip, and Spine," Journal of Biomechanics, vol. 35 , no. 4, pp. 543-548, 2002.

[54] H. Sun, H. Inaoka, Y. Fukuoka, T. Masuda, A. Ishida and S. Morita, "Range of Motion Measurement of an Artificial Hip Joint Using CT Images," Medical \& Biological Engineering \& Computing, vol. 45, no. 12, pp. 1229-1235, 2007.

[55] M. J. Kang, H. Sadri, R. Stern, N. Magnenat-Thalmann, P. Hoffmeyer and H. S. Ji, "Determining the Location of Hip Joint Centre: Application of a Conchoid's Shape to the Acetabular Cartilage Surface of Magnetic Resonance Images," Computer 
Methods in Biomechanics and Biomedical Engineering, vol. 14, no. 1, pp. 65-71, 2011.

[56] C. M. Tylkowski, S. R. Simon and J. M. Mansour, "Internal Rotation Gait in Spastic Cerebral Palsy," in The Hip, 1982.

[57] T. P. Andriacchi, G. B. J. Andersson, R. W. Fermier, D. Stern and J. O. Galante, "A Study of Lower-limb Mechanics during Stair-climbing," The Journal of Bone \& Joint Surgery, vol. 62, no. 5, pp. 749-757, 1980.

[58] A. Cappozzo, F. Catani, U. Della Croce and A. Leardini, "Position and Orientation of Bones during Movement: Anatomical Frame Definition and Determination," Clinical Biomechanics, vol. 10, pp. 171-178, 1995.

[59] T. P. Andriacchi, J. O. Galante and R. W. Fermier, "The Influence of Total Kneereplacement Design on Walking and Stair-climbing," The Journal of Bone \& Joint Surgery, vol. 64, no. 9, pp. 1328-1335, 1982.

[60] A. L. Bell, D. R. Pedersen and R. A. Brand, "A Comparison of the Accuracy of Several Hip Center Location Prediction Methods," Journal of Biomechanics, vol. 23, no. 6, pp. 616-621, 1990.

[61] R. Davis III, S. Ounpuu, D. Tyburski and J. Gage, "A Gait Analysis Data Collection and Reduction Technique," Human Movement Science, vol. 10, pp. 575-587, 1991.

[62] A. Cappozzo, "Gait Analysis Methodology," Human Movement Science, vol. 3, no. 1, pp. 27-50, 1984.

[63] K. M. Shea, M. W. lenhoff, J. C. Otis and S. I. Backus, "Validation of a Method for Location of the Hip Joint Center," Gait \& Posture, vol. 5, no. 2, pp. 157-158, 1997.

[64] S. S. Gamage and J. Lasenby, "New Least Squares Solutions for Estimating the Average Centre of Rotation and the Axis of Rotation," Journal of Biomechanics, vol. 35, no. 1, pp. 87-93, 2002.

[65] N. Chernov and C. Lesort, "Least Squares Fitting of Circles and Lines," Journal of Mathematical Imaging and Vision, vol. 23, no. 3, pp. 239-252, 2005.

[66] P. Deuflhard, Numerical Analysis in Modern Scientific Computing, Springer, 2003.

[67] P. Delonge, "Computer Optimization of Deschamps' Method and Error Cancellation 
in Reflectometry," in In Proc. IMEKO-Symp. Microwave Measurements, Budapest, 1972.

[68] I. Kasa, "A Circle Fitting Procedure and its Error Analysis," IEEE Transactions on Instrumentation and Measurement, vol. 1001, no. 1, pp. 8-14, 1976.

[69] K. Halvorsen, "Bias Compensated Least Squares Estimate of the Center of Rotation," Journal of Biomechanics, vol. 36, no. 7, pp. 999-1008, 2003.

[70] V. Pratt, "Direct Least-squares Fitting of Algebraic Surfaces," Computer Graphics, vol. 21, no. 4, pp. 145-152, 1987.

[71] J. L. Hicks and J. G. Richards, "Clinical Applicability of Using Spherical Fitting to Find Hip Joint Centers," Gait \& Posture, vol. 22, no. 2, pp. 138-145, 2005.

[72] A. Cappozzo, A. Cappello, U. D. Croce and F. Pensalfini, "Surface-marker Cluster Design Criteria for 3-D Bone Movement Reconstruction," IEEE Transactions on Biomedical Engineering, vol. 44, no. 12, pp. 1165-1174, 1997.

[73] M. A. Lafortune and M. J. Lake, "Errors in 3D Analysis of Human Movement," in First International Symposium on 3D Analysis, Montreal, 1991.

[74] D. Karlsson and A. Lundberg, "Accuracy Estimation of Kinematic Data Derived from Bone Anchored External Markers," in Proceedings of the 3rd International Symposium on 3-D Analysis of Human Motion, Stockholm, 1994.

[75] J. Fuller, L. J. Liu, M. C. Murphy and R. W. Mann, "A Comparison of Lowerextremity Skeletal Kinematics Measured Using Skin-and Pin-mounted Markers," Human Movement Science, vol. 16, no. 2, pp. 219-242, 1997.

[76] C. Angeloni, A. Cappozzo, F. Catani and A. Leardini, "Quantification of Relative Displacement between Bones and Skin-and Plate-mounted Markers," in Eighth Meeting of the European Society of Biomechanics, Rome, 1992.

[77] K. Manal, I. McClay, S. Stanhope, J. Richards and B. Galinat, "Comparison of Surface Mounted Markers and Attachment Methods in Estimating Tibial Rotations during Walking: An in Vivo Study," Gait \& Posture, vol. 11, no. 1, pp. 38-45, 2000.

[78] B. A. Maslen and T. R. Ackland, "Radiographic Study of Skin Displacement Errors in the Foot and Ankle during Standing," Clinical Biomechanics, vol. 9, no. 5, pp. 
291-296, 1994.

[79] M. Sati, J. De Guise, S. Larouche and G. Drouin, "Quantitative Assessment of Skinbone Movement at the Knee," The Knee, vol. 3, no. 3, pp. 121-138, 1996.

[80] S. Tashman and W. Anderst, "Skin Motion Artifacts at the Knee during Impact Movements," Gait \& Posture, vol. 16, pp. 11-12, 2002.

[81] R. Stagni, S. Fantozzi, A. Cappello and A. Leardini, "Quantification of Soft tissue Artefact in Motion Analysis by Combining 3D Fluoroscopy and Stereophotogrammetry: A Study on Two Subjects," Clinical Biomechanics, vol. 20, no. 3, pp. 320-329, 2005.

[82] L. Yahia-Cherif and N. Magnenat-Thalmann, "Quantification of Skin Movements Artefacts Using MRI," in 9th International Symposium on 3D Analysis of Human, Valenciennes, 2006.

[83] A. Cappello, A. Cappozzo, P. F. La Palombara, L. Lucchetti and A. Leardini, "Multiple Anatomical Landmark Calibration for Optimal Bone Pose Estimation," Human Movement Science, vol. 16, no. 2, pp. 259-274, 1997.

[84] K. A. Ball and M. R. Pierrynowski, "Modeling of the Pliant Surfaces of the Thigh and Leg during Gait," in In BiOS'98 International Biomedical Optics Symposium, 1998.

[85] L. Lucchetti, A. Cappozzo, A. Cappello and U. D. Croce, "Skin Movement Artefact Assessment and Compensation in the Estimation of Knee-joint Kinematics," Journal of Biomechanics, vol. 31, no. 11, pp. 977-984, 1998.

[86] T. P. Andriacchi, E. J. Alexander, M. K. Toney, C. Dyrby and J. Sum, "A Point Cluster Method for in vivo Motion Analysis: Applied to a Study of Knee Kinematics," Journal of Biomechanical Engineering, vol. 120, no. 6, pp. 743-749, 1998.

[87] C. Joslin, "http://mocap.csit.carleton.ca/," Carleton University, 2010. [Online].

[88] D. A. Christensen, Ultrasonic Bioinstrumentation, New York: John Wiley \& Sons, 1988.

[89] J. L. Prince and J. M. Links, Medical Imaging: Signals and Systems, Upper Saddle 
River: Pearson Prentice Hall, 2006.

[90] F. W. Kremkau, Diagnostic Ultrasound: Principles and Instruments, Missouri: Saunders Elesvier, 2006.

[91] W. R. Hedrick, D. L. Hykes and D. E. Starchman, Ultrasound Physics \& Instrumentation, Missouri: Elsevier Mosby, 2005.

[92] R. M. Ehrig, M. O. Heller, S. Kratzenstein, G. N. Duda, A. Trepczynski and W. R. Taylor, "The SCoRE Residual: A Quality Index to Assess the Accuracy of Joint Estimations," Journal of Biomechanics, vol. 44, no. 7, pp. 1400-1404, 2011.

[93] S. Piazza, A. Erdemir, N. Okita and P. Cavanagh, "Assessment of the Functional Method of Hip joint Center Location Subject to Reduced Range of Hip Motion," Journal of Biomechanics, vol. 37, no. 3, pp. 349-356, 2004. 